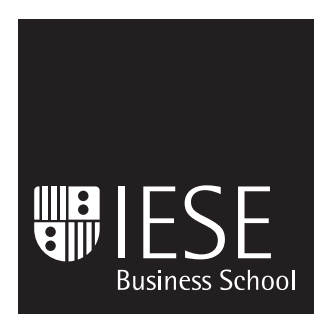

Working Paper

WP no 722

November, 2007

University of Navarra

\title{
THE IMPACT OF QUICK RESPONSE IN INVENTORY-BASED COMPETITION
}

\author{
Felipe Caro ${ }^{1}$ \\ Víctor Martínez de Albéniz ${ }^{2}$
}

${ }^{1}$ Professor, UCLA Anderson School of Management

${ }^{2}$ Professor, Operations Management and Technology, IESE

IESE Business School - University of Navarra

Avda. Pearson, 21 - 08034 Barcelona, Spain. Tel.: (+34) 932534200 Fax: (+34) 932534343

Camino del Cerro del Águila, 3 (Ctra. de Castilla, km 5,180) - 28023 Madrid, Spain. Tel.: (+34) 913570809 Fax: (+34) 913572913

Copyright ${ }^{\odot} 2007$ IESE Business School. 


\title{
The Impact of Quick Response in Inventory-Based Competition
}

\author{
Felipe Caro • Victor Martínez-de-Albéniz \\ UCLA Anderson School of Management 110, Westwood Plaza, Suite B420 Los Angeles, \\ CA 90095, USA \\ IESE Business School University of Navarra Av. Pearson 2108034 Barcelona, Spain \\ fcaro@anderson.ucla.edu•valbeniz@iese.edu
}

October 28, 2007

We propose a multi-period extension of the competitive newsvendor model of Lippman and McCardle (1997) to investigate the impact of quick response under competition. For this purpose, we consider two retailers that compete in terms of inventory: customers that face a stockout at their first-choice store will look for the product at the other store. Consequently, the total demand that each retailer faces depends on the competitor's inventory level. We allow for asymmetric reordering capabilities, and we are particularly interested in the case when one of the firms has a lower ordering cost but can only produce at the beginning of the selling season, whereas the second firm has higher costs but can replenish stock in a quick response manner taking advantage of any incremental knowledge about demand (if it is available). We visualize this problem as the competition between a traditional maketo-stock retailer that builds up inventory before the season starts versus a retailer with a responsive supply chain that can react to early demand information. We provide conditions for this game to have a unique pure-strategy subgame-perfect equilibrium, which then allows us to perform numerical comparative statics. Our results confirm in a competitive setting the intuitive fact that quick response is more beneficial when demand uncertainty is higher, or exhibits a higher correlation over time. Finally, we find that part of the competitive advantage from quick response arises from the asymmetry in response capabilities.

\section{Introduction}

In recent years, the apparel industry has seen the rise of what has been called fast fashion retailers. These are clothing companies that are able to respond quickly to market trends and introduce new products very frequently. Most of these products have a life-cycle of no more than a few weeks, and by the time the cycle is over, they are promptly replaced by a more "fashionable" item. The high assortment rotation has become a distinctive feature that has increased the average number of visits to the store, since now customers are aware that they can always find something new. 
In Europe, where the concept began, fast fashion has been denominated a 21st-century retailing phenomenon with representative companies such as $\mathrm{H} \& \mathrm{M}$ and the Inditex group, owner of Zara (Davidson 2005). Besides their natural talent to design on-trend clothes, a crucial part of their success is due to their flexible supply chain and operational competencies (Ghemawat and Nueno 2003). In particular, fast fashion retailers can make in-season replenishments thanks to remarkably low lead times, in the order of weeks rather than months. The latter is achieved in most cases through local production or expediting, which obviously translates into higher unit costs.

In the case of North America, fast fashion remains a niche that represents no more than two percent of the apparel business (Foroohar 2006). Large clothing retailers like The Gap seem too big and might not have the incentives to restructure their entire supply chain to mimic their European competitors since their customers have been historically less fashionforward. They might however borrow a few elements of fast fashion. For example, they can strength the link with their suppliers, or they can move the production of trendier items to Mexico instead of Asia in order to shorten lead times. The extent to which traditional retailers should adopt or convert to fast fashion remains an open question and serves as part of the motivation for this paper.

Despite the incipient (but growing) success of fast fashion in North America, the concept itself builds upon quick response (QR), which was an apparel manufacturing initiative that started primarily in the United States during the mid 1980s (Hammond and Kelly 1990). The main objective of $\mathrm{QR}$ is to drastically reduce lead times and setup costs to allow the postponement of ordering decisions until right before (or during) the retail selling season when better demand information might be available. A successful implementation of $\mathrm{QR}$ is typically based on the effective use of information technologies. Fast fashion has taken QR to a higher level and has leveraged on the minimal lead times by introducing new products on a regular basis, and therefore enabling a dynamic assortment that basically fulfills the ideal of providing "fashion on demand".

The overall success of fast fashion is attributable to a combination of multiple factors. The interaction between all the elements involved is at a preliminary stage of being understood. There has been extensive qualitative work that describes the different cases or examples of fast fashion companies. However, the academic literature on this topic remains scarce. In this paper we aim at understanding the impact of one specific element of fast fashion. Namely, the QR component that is arguably the basis for all the other elements that later come into play. Therefore, we disregard assortment, pricing or market positioning decisions 
related to fast fashion, and we focus on the essential capability of having more flexibility in terms of inventory replenishment. We look at the problem in a competitive setting, since from its inception, QR advocates have claimed that it is the only viable strategy under the current conditions in the apparel market, similar to what just-in-time manufacturing has meant to the auto industry (Hammond and Kelly 1990).

We consider a model with two retailers selling a substitutable product over a finite horizon. The two retailers compete based on inventory. When a stockout occurs at one retailer, the unsatisfied customer walks into the second retailer, where she is served if stock is available. Thus, the inventory decision of a given retailer depends on the level of inventory at the competitor. Moreover, as time goes by, the retailers can incorporate any additional demand information into their stocking decisions. The model allows for asymmetric retailers and we analyze their competitive strategies, considering the equilibrium inventory decisions. Our model can be seen as a multi-period extension of the competitive newsboy developed by Lippman and McCardle (1997). As in their case, we are interested in determining and characterizing the existence of a unique pure-strategy subgame-perfect equilibrium, which then allows us to understand and compare the outcomes for each retailer, and in particular assess the potential benefits of implementing QR.

Our paper makes contributions to the operations literature from both the methodological and managerial standpoint. From a methodological perspective, we solve an asymmetric multi-period inventory-based competition model, where the asymmetry is in terms of reordering and demand learning capabilities. ${ }^{1}$ We are not aware of any other paper that studies dynamic horizontal inventory-based competition with demand correlation over time. Several authors have previously studied the infinite horizon case, but under such conditions that it reduces to a myopic single-period problem. On the contrary, we formulate a dynamic finite horizon model, and we analyze four different cases. For each one of them, we provide sufficient conditions that guarantee the existence of a unique equilibrium. The conditions are summarized in Table 1 (the meaning of each assumption is discussed later). Note that we pay special attention to the two-period case since it is the most common approach that has been used in the literature to model QR (see, for instance, section 10.4 in Cachon and Terwiesch 2005). Also, our results extend Theorem 3 of Lippman and McCardle (1997) in two ways: (i) to asymmetric retailers in the single-period case, and (ii) to an arbitrary number of periods with information updates in the symmetric case (under a linear demand split). We also allow for arbitrary initial inventory levels in both cases.

\footnotetext{
${ }^{1}$ Asymmetric net margins and demand splitting functions are also allowed.
} 
From a managerial standpoint, this paper is a first attempt at trying to understand what the competitive advantage of fast fashion is compared to more traditional retailing operations. As mentioned before, our analysis is restricted to the benefits that stem from QR. In particular, we provide a detailed numerical study where we compare the profits in a competition of two slow response (SR) firms, with those achieved in a competition where one or both retailers have implemented QR. Our results indicate that both retailers are better off in the QR vs. SR competition compared to the SR vs. SR case. In addition, we show that part of the competitive advantage for a $\mathrm{QR}$ retailer comes from the asymmetry, i.e., from being faster than the competitor, and these benefits are larger under higher demand uncertainty or higher correlation over time.

\begin{tabular}{|c|c|c|c|c|}
\hline Assumption & $\begin{array}{l}\text { Demand signal } \\
\qquad \mathbf{T}=\mathbf{2}\end{array}$ & $\begin{array}{c}\text { Mid-season replenishment } \\
\mathbf{T}=\mathbf{2}\end{array}$ & $\begin{array}{l}\text { Passive competitor } \\
\qquad \mathbf{T} \geq \mathbf{2}\end{array}$ & $\begin{array}{c}\text { Symmetric retailers } \\
\qquad \mathbf{T} \geq \mathbf{2}\end{array}$ \\
\hline Margins & Different costs per period & Non-increasing & Non-increasing & - \\
\hline Infinite support & - & Only in initial period & In all periods but last & - \\
\hline Log-concavity & - & Only in initial period & In all periods but last & - \\
\hline Independence & - & $\begin{array}{c}\left(D_{1}-k D_{2}\right) \text { independent of } \\
D_{2} \text { for some } k \geq 0\end{array}$ & Across all periods & - \\
\hline Likelihood order & - & Non-decreasing & Non-decreasing & - \\
\hline Linear split & - & Only in last period if $k>0$ & - & In all periods \\
\hline
\end{tabular}

Table 1: Sufficient conditions for a unique pure-strategy subgame-perfect equilibrium.

The remainder of the paper has the following structure. In section $\S 2$ we review the existing literature, mostly on inventory-based competition models. Then, in $\S 3$ we develop our model, and in $\S 4$ we establish the existence and uniqueness of a pure-strategy subgameperfect equilibrium for the four different cases mentioned in Table 1 . In $\S 5$ we perform numerical comparative statics in order to understand how the equilibrium depends on the parameters of the model. Finally, in the last section $\S 6$ we conclude and discuss future research directions. All the proofs are available in the technical appendix, unless otherwise noted.

\section{Literature Review}

Fast fashion has been discussed extensively in the popular press, see for instance The Economist (2005). In more academic terms, the literature is mostly descriptive with an emphasis on the qualitative aspects of the retailing strategy. Many cases have been written, in particular for the Spanish company Zara, e.g., Ghemawat and Nueno (2003), McAfee et 
al (2004), and Ferdows et al (2004). From a quantitative perspective, during the 1990s significant progress was made in understanding the impact of QR in an isolated supply chain, mostly in a two-period setting (see Fisher and Raman 1996, Iyer and Bergen 1997, and references therein). More recently, Cachon and Swinney (2007) have looked at QR in the presence of strategic customers. All this work is related to ours because we focus on the $\mathrm{QR}$ aspect of fast fashion. However, as mentioned before, fast fashion goes beyond QR, in particular by introducing a large number of new products during the retail selling season. In that respect, Caro and Gallien (2007) provide a closed-form policy for one of the distinctive operational challenges faced by fast fashion firms, namely the dynamic assortment problem

To the best of our knowledge, there has not been much analytical work that tries to identify the drivers of fast fashion's success in a competitive context. Clearly, the answer is not simple since there are many interweaved factors that come into play. For that reason, as a first attempt to understand the (potential) competitive advantage, in this paper we focus exclusively on the QR capability and we openly disregard other important elements of fast fashion. With this scope in mind, we are left with an inventory-based competition problem for substitutable products. Several models have been developed in the literature for this problem. In Table 2 we provide a (non-comprehensive) summary of preceding work. Our paper contributes to this stream of research by solving a multi-period model that allows for asymmetric retailers in terms of reordering and demand learning capabilities.

In the single-period case with $n$ retailers, Lippman and McCardle (1997) prove the existence of a pure Nash equilibrium under the general assumption that the effective demand faced by a particular firm is stochastically decreasing in the inventory levels of the other firms, which comes naturally in the case of substitutable products, see Netessine and Zhang (2005). The existence of a unique Nash equilibrium requires additional assumptions as those listed in the last column of Table 2.

In the infinite horizon case, several authors have shown that, under suitable conditions, there exists a Nash equilibrium in which each retailer follows a stationary base-stock policy. All these results stem from the dynamic oligopoly model by Kirman and Sobel (1974). In practice, this is equivalent to solving a single-period problem. Note that even if the latter has a unique Nash equilibrium, that does not guarantee a unique subgame-perfect equilibrium in the multi-period case.

Several other (retailing) competition models in which inventories play an important role have been studied in the literature. For instance, in an infinite horizon setting, Li (1992) looks at delivery-time competition and shows that when all retailers are identical they tend 
to make-to-stock. Anupindi and Bassok (1999) consider inventory-based competition à la Parlar (1988), and study the impact of "market search" (i.e., the spill-over fraction) on the manufacturer's profit. Bernstein and Federgruen (2004) examine the case of retailers that compete on price and then set their inventory levels accordingly. Gaur and Park (2007) consider customers sensitive to negative experiences such as a stockout, and study the competition of retailers on the basis of their service levels. As before, given the stationary model formulation in these papers, the solution is myopic in the sense of Sobel (1981) and the analysis reduces to a single-period problem.

\begin{tabular}{|c|c|c|c|c|c|c|c|c|}
\hline Author/Year & $\begin{array}{l}\text { Num. } \\
\text { Firms }\end{array}$ & $\begin{array}{l}\text { Num. } \\
\text { Periods }\end{array}$ & $\begin{array}{l}\text { Myopic } \\
\text { Solution }\end{array}$ & $\begin{array}{l}\text { Back- } \\
\text { logging }\end{array}$ & Stochastic Demand Model & $\begin{array}{l}\text { Demand } \\
\text { Learning }\end{array}$ & $\begin{array}{l}\text { Typ e of Asymmetric } \\
\text { Retailers Allow ed }\end{array}$ & $\begin{array}{l}\text { Sufficient Cond itions for } \\
\text { the Existence of a Unique } \\
\text { Nash Eq uilib rium }\end{array}$ \\
\hline Par1ar(1988) & 2 & 1 & Yes & $\begin{array}{c}\text { Not } \\
\text { allowed }\end{array}$ & $\begin{array}{l}\text { Independent firm demands are } \\
\text { aggegated to industry dem and. } \\
\text { Determ inistic spillovers. }\end{array}$ & $\begin{array}{c}\text { Not } \\
\text { allowed }\end{array}$ & $\begin{array}{l}\text { Interms of cost } \\
\text { parameters and } \\
\text { effective demand. }\end{array}$ & $\begin{array}{l}\text { Continuous and strictly } \\
\text { increasing cumulative } \\
\text { demand. }\end{array}$ \\
\hline $\begin{array}{l}\text { Lippman and } \\
\text { McC ardle (1997) }\end{array}$ & $\mathrm{N}$ & 1 & Yes & $\begin{array}{l}\text { Not } \\
\text { allowed }\end{array}$ & $\begin{array}{l}\text { A ggregate inchastry dem and is } \\
\text { allocated across firms. Gener al } \\
\text { splitting rules are considered. }\end{array}$ & $\begin{array}{c}\text { Not } \\
\text { allowed }\end{array}$ & As in Parlar (1988). & $\begin{array}{c}\text { Parlar (1988) + symm etric } \\
\text { costs \& revenues + strictly } \\
\text { increasing deterministic } \\
\text { dem and split. }\end{array}$ \\
\hline $\begin{array}{l}\text { Mahajan and van } \\
\text { Ryzin (2001) }\end{array}$ & $\mathrm{N}$ & 1 & Yes & $\begin{array}{c}\mathrm{N} \text { ot } \\
\text { allowed }\end{array}$ & $\begin{array}{l}\text { Based on utility maximization. } \\
\text { Custom ers can buy more than } \\
\text { one item. }\end{array}$ & $\begin{array}{c}\text { Not } \\
\text { allowed }\end{array}$ & As in Parlar (1988). & $\begin{array}{c}\text { Symm etric retailers }+ \\
\text { incomplete/nontrivial dem and } \\
\text { diversion + continuous } \\
\text { demand. }\end{array}$ \\
\hline $\begin{array}{l}\text { Avsar and } \\
\text { Baykal-G ür soy } \\
\text { (2002) }\end{array}$ & 2 & $\infty$ & Yes & $\begin{array}{c}\text { Not } \\
\text { allowed }\end{array}$ & As in Parlar (1988). & $\begin{array}{c}\text { Not } \\
\text { allowed }\end{array}$ & As in Parlar (1988). & $\begin{array}{l}\text { Continuous dem and }+ \\
\text { stationary policies }+1 \text { ow } \\
\text { initial dem and. }\end{array}$ \\
\hline $\begin{array}{l}\text { Netessine and } \\
\text { Rudi(2003) }\end{array}$ & $\mathrm{N}$ & 1 & Yes & $\begin{array}{c}\text { Not } \\
\text { allowed }\end{array}$ & $\begin{array}{l}\text { As in Parlar (1988) but } \\
\text { correlations are allowed }\end{array}$ & $\begin{array}{l}\text { Not } \\
\text { allowed }\end{array}$ & As in Parlar (1988). & $\begin{array}{l}\text { Continuous dem and }+ \\
\text { incomplete dem and diver sion. }\end{array}$ \\
\hline $\begin{array}{l}\text { Netessine, Rudi } \\
\text { and Wang }(2005)\end{array}$ & 2 & $1, \infty$ & Yes & Allowed & $\begin{array}{c}\text { Model I: identical to N etessine } \\
\text { and Rudi (2003). Models II-IV: } \\
\text { allow backor ders. }\end{array}$ & $\begin{array}{c}\text { Not } \\
\text { allowed }\end{array}$ & As in Parlar (1988). & $\begin{array}{c}\text { Same as in Avsar andB aykal- } \\
\text { Gür soy (2002). }\end{array}$ \\
\hline $\begin{array}{l}\text { Nagarajan and } \\
\text { Rajagopalan } \\
(2005)\end{array}$ & 2 & $\mathrm{~T}$ & No & Allowed & $\begin{array}{l}\text { Total demand is determ inistic } \\
\text { but allocation is random. }\end{array}$ & $\begin{array}{c}\text { Not } \\
\text { allowed }\end{array}$ & As in Parlar (1988). & $\begin{array}{l}\text { N ew sboy ratio equal to } 0.5 \text { or } \\
\text { more (then each retailer can } \\
\text { ignore its competitor). }\end{array}$ \\
\hline Present paper & 2 & $\mathrm{~T}$ & No & $\begin{array}{l}\mathrm{N} \text { ot } \\
\text { allowed }\end{array}$ & $\begin{array}{l}\text { As in Lippm an and McC ar dle } \\
\text { (1997) with a strictly increasing } \\
\text { deterministic demand split. }\end{array}$ & Allowed & $\begin{array}{c}\text { As in } \mathrm{P} \text { arlar (1988) }+ \\
\text { asym metric reor dering } \\
\text { and dem andlearning } \\
\text { capabilities. }\end{array}$ & See T able 1 above. \\
\hline
\end{tabular}

Table 2: Inventory-based competition models for substitutable products.

Given the extremely short life-cycle of fashionable clothing, finite horizon models seem more appropriate. In that matter, the work by Hall and Porteus (2000) is conceptually close to ours because, despite the fact that they consider competition based on customer service instead of (non-perishable) inventory and information updates are not allowed, the retailers can only take actions to prevent leakage of demand to the competitor rather than proactively attract demand to itself. Under these conditions together with a multiplicative demand model, they are able to show the existence of a unique subgame-perfect equilibrium. Liu et al (2007) extend the result to a more general demand model that includes the additive case. Olsen and Parker (2006) provide an alternative extension in which the retailers can 
hold inventory over time and can advertise to attract dissatisfied customers from its competitor's market. The existence of a unique equilibrium is guaranteed by assuming a particular salvage value function and low initial inventory levels (as in Avsar and Baykal-Gürsoy 2002). Interestingly, in equilibrium, the game effectively becomes two parallel Markov decision processes where each firm can make stocking decision independent of the other firm's choices. We obtain a similar result for the case of two symmetric retailers, though under a different set of assumptions.

\section{A Multi-Period Inventory Competition Model}

In this section we formulate the multi-period inventory competition model that will be used later to study the benefits of QR. In $\S 3.1$ we present the basic features and assumptions. Then in $\S 3.2$, we introduce the dynamic aspect of the model and the solution approach (i.e., sub-game perfection).

\subsection{Basic Features and Assumptions}

In what follows, we present each one of the assumptions that lead to our multi-period model. The assumptions appear in italics and an explanation or discussion follows whenever appropriate. Any related notation is introduced as well.

(A1) There are only two firms that sell substitute products, and each firm maximizes the total expected profits over a finite horizon of $T$ periods.

Let indices $i$ and $t$ denote retailers and periods respectively, with $i=1,2$ and $t=T, \ldots, 1$. Note that periods are counted backwards, and we consider a finite horizon to represent the short product life-cycle in the fashion apparel industry. The index $j$ is also reserved to denote a retailer, and throughout the paper it is understood that $i \neq j$. Since the retailers sell perfect substitutes, a customer that cannot find the product at her preferred retailer will check if it is available at the competitor.

(A2) The aggregate customer demand in period $t$ (denoted $D_{t}$ ) is continuous, stochastic, and may be correlated across periods.

Let $f_{t}$ be the p.d.f. of the demand in period $t, F_{t}$ its c.d.f., $\bar{F}_{t} \equiv 1-F_{t}$, and $\bar{F}_{t}^{-1}$ its inverse. When there is correlation across time, we denote the p.d.f. and c.d.f. of the demand 
in period $t$ as $f_{t \mid I_{t}}$ and $F_{t \mid I_{t}}$ respectively, where $I_{t}$ is the demand information available at that point in time, which is assumed to be common knowledge. Typically, the information would be the vector of past demand realizations (that is, $\left\{D_{t+1}, D_{t+2}, \ldots, D_{T}\right\}$ ) and/or any data or demand signal that has become available. An important remark is that we do not allow the information vector to be a function of the decisions in the current period. In other words, information is uncensored, just as in most QR models.

(A3) In a given period $t$, the effective demand faced by retailer $i$ is composed of two parts: (i) the original demand, and (ii) the overflow demand. The original demand is expressed as $q_{t}^{i}\left(D_{t}\right)$, where $q_{t}^{i}$ is the (initial) allocation function (also referred to as the demand splitting function) which is assumed to be strictly increasing, and we have that $D_{t}=q_{t}^{1}\left(D_{t}\right)+q_{t}^{2}\left(D_{t}\right){ }^{2}$

The original demand is made of customers that naturally choose retailer $i$ over the competitor $j$, and the overflow demand is made of those customers that initially choose $j$ but end up buying at $i$ because $j$ runs out of stock. This overflow demand is equal to

$$
\max \left\{0, q_{t}^{j}\left(D_{t}\right)-y_{t}^{j}\right\}
$$

where $y_{t}^{j}$ is firm $j$ 's inventory level (after replenishment) in period $t{ }^{3}$ Then the effective (realized) demand faced by retailer $i$ is given by $R_{t}^{i}\left(y_{t}^{j}\right) \equiv q_{t}^{i}\left(D_{t}\right)+\max \left\{0, q_{t}^{j}\left(D_{t}\right)-y_{t}^{j}\right\}$. Since this is a key assumption in our model, several important observations follow:

- The effective demand $R_{t}^{i}\left(y_{t}^{j}\right)$ depends only on the competitor's inventory level. Therefore, competition is based on the inventory levels, but retailer $i$ can only limit the customers it loses rather than influence those it gains. In an authentic fast fashion setting, a retailer would typically attract more demand by sustaining a high assortment rotation. Our model does not consider such feature since we focus on understanding the impact of the QR capability for one particular product. We also note that, except for Netessine et al (2006), all the papers mentioned in Table 2 consider competitive models in which the retailer can only prevent leakage rather than attract additional demand. The same happens in Hall and Porteus (2000) and Liu et al (2007).

\footnotetext{
${ }^{2}$ In the demand signal case studied in $\S 4.2 .1$, since there is no demand realization in the first period, we let $q_{2}^{i}=0, i=1,2$.

${ }^{3}$ Our model can be directly extended to the case of imperfect substitution. That is, when only a fraction $\delta$ of customer choose to substitute when they face a stockout. It suffices to multiply Equation (1) by $\delta$, and all the equilibrium results follow through.
} 
- In Parlar (1988) and other similar papers, independent firm demands are aggregated into industry demand. On the contrary, in Lippman and McCardle (1997) and its successors, aggregate industry demand is allocated across firms. If the allocation is deterministic, then in each period there is only one source of uncertainty, namely, the total demand $D_{t}$. We have followed the latter approach since we believe it represents better the case of fashionable items in which the main uncertainty is the size of the market (i.e., how well a product will sell) rather than the initial allocation across retailers. It is worth noting that our results for two periods $(T=2)$ can be extended to the case when the initial allocation depends on the current demand information $I_{t}$.

- As in the proof of uniqueness by Lippman and McCardle (1997), we require the initial allocation function $q_{t}^{i}$ to be strictly increasing in $D_{t}$. It must be strictly monotone because we need the inverse $\left(q_{1}^{i}\right)^{-1}$ to be well defined, and it must be increasing because our analysis requires that once the stocking decisions have been made, the retailer that runs out of stock first is the same one under all possible demand scenarios (in a given period). These conditions implicitly impose a positive correlation between the original demands of firms 1 and 2. Again, this is reasonable when the main source of uncertainty is the market size. Note that the correlation can be anywhere between 0 to 1 . It is perfect (equal to one) for the linear demand splitting case, but can be close to zero as well. ${ }^{4}$ Some models in the literature, e.g., Nagarajan and Rajagopalan (2005), assume negative correlation between the original demands of the two firms, which is an appropriate assumption when the retailers are competing for a fixed pool of customers. Another class of models assume that the (effective) demand allocation is proportional to the individual stocking levels (see Cachon 2003). In that case, demands at the retailers are perfectly correlated, and a retailer that stocks more will get more. This goes back to the previous discussion about the retailer being able to influence its effective demand, but also has the inconvenience of making the multi-period analysis untractable.

(A4) At the beginning of period $t$ both retailers decide simultaneously the order-up-to levels $\left(y_{t}^{1}, y_{t}^{2}\right)$ based on the initial stock levels $\left(x_{t}^{1}, x_{t}^{2}\right)$ and the demand information $I_{t}$.

\footnotetext{
${ }^{4}$ Consider the following example: $q_{t}^{1}\left(D_{t}\right)=0$ for $0 \leq D_{t} \leq a$ and $q_{t}^{2}\left(D_{t}\right)=a$ when $a \leq D_{t} \leq 1$, and $D_{t}$ is uniform in $[0,1]$. Then $\operatorname{Cov}\left(q_{t}^{1}\left(D_{t}\right), q_{t}^{2}\left(D_{t}\right)\right)=\frac{a^{2}(1-a)^{2}}{4}, \operatorname{Var}\left(q_{t}^{1}\left(D_{t}\right)\right)=\frac{(1-a)^{3}(1+3 a)}{12}$ and $\operatorname{Var}\left(q_{t}^{2}\left(D_{t}\right)\right)=\frac{a^{3}(4-3 a)}{12}$. Hence, $\operatorname{Corr}\left(q_{t}^{1}\left(D_{t}\right), q_{t}^{2}\left(D_{t}\right)\right)=\frac{3 \sqrt{a} \sqrt{1-a}}{\sqrt{1+3 a} \sqrt{1+3(1-a)}}$, which is close to 0 for $a \approx 0$ or $a \approx 1$.
} 
The status of both retailers at the beginning of a given period $t$ is described by the initial stock levels $\left(x_{t}^{1}, x_{t}^{2}\right)$, and the status of the market in which the retailers compete is described by the current demand information $I_{t}$. Then, the state of the system (i.e, the retailers and the market) is given by the vector $\left(x_{t}^{1}, x_{t}^{2}, I_{t}\right)$ and we assume that the retailers decide their actions, i.e. the order-up-to levels $\left(y_{t}^{1}, y_{t}^{2}\right)$, contingent on the state. In other words, the retailers play Markovian strategies (see Fudenberg and Tirole 1991).

(A5) The unit cost and price for retailer $i$ in period $t$ are constant parameters denoted $c_{t}^{i}$ and $p_{t}^{i}$ respectively. The retailers are said to be symmetric if they have the same cost and price in all periods.

We exclude pricing decisions from the model. This allows us to focus on the use of inventory as a competitive lever. This assumption is consistent with the fact that fast fashion retailers rely less on markdowns, see Ghemawat and Nueno (2003). Note that if $c_{t}^{j} \geq p_{t}^{j}$, then retailer $j$ will not order in period $t$. Therefore, by choosing the appropriate cost and price parameters we can model the situation in which firm $j$ has a lower ordering cost but is only allowed to produce at the beginning of the selling season, whereas retailer $i$ has higher costs but can replenish stock every period taking advantage of any incremental knowledge about demand (if it is available). This represents the case of two retailers that are asymmetric in terms of reordering and demand learning capabilities.

(A6) We ignore holding and lost sales penalty costs, and there is no minimum ordering quantity.

Overall, we aim at formulating a parsimonious model. We neglect inventory holding costs, as they are less relevant for short life-cycle products. However, these costs can also be incorporated in the model. The same holds for the lost sales penalty costs. ${ }^{5}$ On the contrary, the QR problem with minimum ordering quantities lies beyond the scope of this paper.

(A7) Leftover inventory can be carried over to the next period, and is lost at the end of the season. If both retailers stockout in a given period, the unsatisfied demand is lost as well.

\footnotetext{
${ }^{5}$ As a matter of fact, if one wished to incorporate lost sales penalty costs, one would add a term $v_{t}^{i} \mathbb{E}\left\{R_{t}^{i}-\right.$ $\left.y_{t}^{i}\right\}^{+}=v_{t}^{i} \mathbb{E}\left\{R_{t}^{i}\right\}-v_{t}^{i} \mathbb{E} \min \left\{y_{t}^{i}, R_{t}^{i}\right\}$ to the revenue; to incorporate the inventory holding cost, one would add a term $h_{t}^{i} \mathbb{E}\left\{y_{t}^{i}-R_{t}^{i}\right\}^{+}=h_{t}^{i} y_{t}^{i}-h_{t}^{i} \mathbb{E} \min \left\{y_{t}^{i}, R_{t}^{i}\right\}$. Hence, we would replace the revenue minus the purchasing $\operatorname{costs} p_{t}^{i} \mathbb{E} \min \left\{R_{t}^{i}, y_{t}^{i}\right\}-c_{t}^{i}\left(y_{t}^{i}-x_{t}^{i}\right)$ by $\left(p_{t}^{i}+v_{t}^{i}+h_{t}^{i}\right) \mathbb{E} \min \left\{R_{t}^{i}, y_{t}^{i}\right\}-v_{t}^{i} \mathbb{E}\left\{R_{t}^{i}\right\}-\left(c_{t}^{i}+h_{t}^{i}\right) y_{t}^{i}-c_{t}^{i} x_{t}^{i}$.
} 
A salvage value could be easily incorporated in the model. Similarly, a straightforward extension allows for backlogged demand to be share between the firms in a deterministic way. Finally, under assumptions (A1)-(A7), the immediate expected profit of retailer $i$ in period $t$ is equal to expected revenues $p_{t}^{i} \mathbb{E}\left\{\min \left\{y_{t}^{i}, R_{t}^{i}\left(y_{t}^{j}\right)\right\}\right\}$, minus purchasing costs $c_{t}^{i}\left(y_{t}^{i}-x_{t}^{i}\right)$, and the leftover inventory is equal to $\left(y_{t}^{i}-R_{t}^{i}\left(y_{t}^{j}\right)\right)^{+}$.

\subsection{Sub-Game Perfect Strategies}

Since the overflow demand depends on the inventory level of the competitor, see Equation (1), we must use game-theoretical tools to analyze the replenishment decision. For expositional purposes, we initially consider the case when the demand information $I_{t}$ is void, and we begin with the terminal period $t=1$. Throughout the paper, when it is clear from the context, we omit the arguments of a given function.

First, let $r_{1}^{i}$ be firm $i$ 's unconstrained expected profit, which can be expressed as

$$
r_{1}^{i}\left(y_{1}^{i}, y_{1}^{j}\right)=\mathbb{E}\left\{-c_{1}^{i} y_{1}^{i}+p_{1}^{i} \min \left\{y_{1}^{i}, R_{1}^{i}\left(y_{1}^{j}\right)\right\}\right\} .
$$

Second, if firm $i$ knew firm $j$ 's order-up-to level $y_{1}^{j}$, then firm $i$ 's best response would come from maximizing expected profits taking into account its initial stock $x_{1}^{i}$ and the competitor's action. In other words, retailer $i$ would solve:

$$
\max _{y_{1}^{i} \geq x_{1}^{i}} c_{1}^{i} x_{1}^{i}+r_{1}^{i}\left(y_{1}^{i}, y_{1}^{j}\right)
$$

Since the term $c_{1}^{i} x_{1}^{i}$ in Equation (3) is constant, firm $i$ 's best response actually comes from maximizing $r_{1}^{i}\left(y_{1}^{i}, y_{1}^{j}\right)$ subject to $y_{1}^{i} \geq x_{1}^{i}$. If the solution to this optimization problem is unique, then we can define the best-response function $b_{1}^{i}\left(x_{1}^{i}, y_{1}^{j}\right)=\operatorname{argmax}_{y_{1}^{i} \geq x_{1}^{i}}\left\{r_{1}\left(y_{1}^{i}, y_{1}^{j}\right)\right\}$ as the optimal stocking level in period 1 for firm $i$ in response to a level $y_{1}^{j}$ from firm $j$, starting with a position of $x_{1}^{i}$.

Of course, in reality, firm $i$ does not know what level of $y_{1}^{j}$ firm $j$ will select. Thus, we use the notion of Nash equilibrium. In our setting, a Nash equilibrium, if it exists, is given by two functions $e_{1}^{1}$ and $e_{1}^{2}$ that might depend on the initial stock levels $\left(x_{1}^{1}, x_{1}^{2}\right)$, and are such that $b_{1}^{1}\left(x_{1}^{1}, e_{1}^{2}\right)=e_{1}^{1}$ and $b_{1}^{2}\left(x_{1}^{2}, e_{1}^{1}\right)=e_{1}^{2}$. Put differently, no player is better off by unilaterally deviating from the equilibrium. We can define the equilibrium expected profit $\pi_{1}^{i}$ by replacing the equilibrium actions in the objective function of Equation (3) to obtain

$$
\pi_{1}^{i}\left(x_{1}^{i}, x_{1}^{j}\right)=c_{1}^{i} x_{1}^{i}+r_{1}^{i}\left(e_{1}^{i}\left(x_{1}^{i}, x_{1}^{j}\right), e_{1}^{j}\left(x_{1}^{j}, x_{1}^{i}\right)\right)
$$


If we now consider a multi-period setting, the notion of Nash equilibrium extends to subgame-perfect equilibrium. An equilibrium is subgame-perfect if it induces a Nash equilibrium (as defined above) in each subgame of the original game (see Fudenberg and Tirole 1991). In our context, a subgame corresponds to a game that is similar to the original one but with one period less to go (i.e., the last period is a subgame of the two-period game, and in turn, the latter is a subgame of the three-period game, and so on and so forth) Therefore, in period $t$ we can construct the best response functions just as we did for the last period, but the only caveat is that now the expected profit is the sum of the immediate profit plus the future profit-to-go, and the latter must be the equilibrium profits of the game with one period less. Formally, the unconstrained expected profit in period $t$ is given by

$$
r_{t}^{i}\left(y_{t}^{i}, y_{t}^{j}\right)=\mathbb{E}\left\{\begin{array}{c}
-c_{t}^{i} y_{t}^{i}+p_{t}^{i} \min \left\{y_{t}^{i}, R_{t}^{i}\left(y_{t}^{j}\right)\right\} \\
+\pi_{t-1}^{i}\left(\left(y_{t}^{i}-R_{t}^{i}\left(y_{t}^{j}\right)\right)^{+},\left(y_{t}^{j}-R_{t}^{j}\left(y_{t}^{i}\right)\right)^{+}\right)
\end{array}\right\},
$$

where $\pi_{t-1}^{i}$ is the equilibrium expected profit of the subgame that starts in period $t-1$. The best response functions are the same as before, i.e.,

$$
b_{t}^{i}\left(x_{t}^{i}, y_{t}^{j}\right)=\operatorname{argmax}_{y_{t}^{i} \geq x_{t}^{i}}\left\{r_{t}\left(y_{t}^{i}, y_{t}^{j}\right)\right\}
$$

and a Nash equilibrium in period $t$, if it exists, is given by two functions $e_{t}^{1}$ and $e_{t}^{2}$ that might depend on the initial stock levels $\left(x_{t}^{1}, x_{t}^{2}\right)$, and are such that $b_{t}^{1}\left(x_{t}^{1}, e_{t}^{2}\right)=e_{t}^{1}$ and $b_{t}^{2}\left(x_{t}^{2}, e_{t}^{1}\right)=e_{t}^{2}$. In order to close the loop, if we want to verify the existence, and then compute a (subgameperfect) equilibrium in period $t+1$, we would need the expected equilibrium profits in period $t$, which is given by

$$
\pi_{t}^{i}\left(x_{t}^{i}, x_{t}^{j}\right)=c_{t}^{i} x_{t}^{i}+r_{t}^{i}\left(e_{t}^{i}\left(x_{t}^{i}, x_{t}^{j}\right), e_{t}^{j}\left(x_{t}^{j}, x_{t}^{i}\right)\right)
$$

and the procedure repeats itself by replacing $t$ with $t+1$ in Equation (5). ${ }^{6}$

The previous definitions were given for the case when the demand information $I_{t}$ is void. The latter would be valid if the demand across periods were independent. However, if the retailers can use current or past demand information to predict future demand, then their actions, and consequently the competitive equilibrium, will be contingent on the information that is actually available. Therefore, in that case, we replace the subindex $t$ with $t \mid I_{t}$ in $r_{t}^{i}, b_{t}^{i}, e_{t}^{i}$, and $\pi_{t}^{i}$, and all the expectations are conditional on $I_{t}$. For instance, instead of $r_{t}^{i}\left(y_{t}^{i}, y_{t}^{j}\right)$ we write $r_{t \mid I_{t}}^{i}\left(y_{t}^{i}, y_{t}^{j}\right)$, and the expectation on the right hand side of Equation (5) is conditional on $I_{t}$.

\footnotetext{
${ }^{6}$ In game theory, this procedure is known as backwards induction (see Fudenberg and Tirole 1991).
} 


\section{Existence and Uniqueness of Equilibrium}

We now present the structural results of the paper. Our goal in this section is to prove the existence of a unique pure-strategy subgame-perfect equilibrium for the four cases mentioned in Table 1. These result have theoretical value, but also allow us to understand the benefits of QR by computing comparative statics of the unique equilibrium (see $§ 5$ ).

We start by considering the single-period problem in $\S 4.1$. Then, in $\S 4.2$ we consider the two-period case and we study the two most cited QR models in literature. Namely, the demand signal and the mid-season replenishment models. ${ }^{7}$ The more general case with two or more periods is studied in $§ 4.3$. There, we analyze the situation when one competitor is passive and when both retailers are symmetric. Note that in Table 1 the mid-season replenishment and the passive competitor cases are grouped together because they have similar proofs.

\subsection{Single-Period Case $(T=1)$}

The single-period problem is an essential building block in our model. To simplify the exposition we omit the dependence on the demand information vector $I_{1}$, but all the discussion throughout this section remains valid if we replace the subindex $t=1$ with with $1 \mid I_{1}$, and all the expectations are conditional on $I_{1}$.

We first consider the unconstrained problem. That is, the version of the problem in which the initial inventory levels are equal to zero. From Equation (2), it is clear that $r_{1}^{i}$ is concave in $y_{1}^{i}$, for all $y_{1}^{j}$. Thus, the optimal inventory policy is a base-stock policy with target level $s_{1}^{i}\left(y_{1}^{j}\right)$, which can be obtained from the first-order conditions $\operatorname{Pr}\left(R_{1}^{i}\left(y_{1}^{j}\right) \geq s_{1}^{i}\left(y_{1}^{j}\right)\right)=c_{1}^{i} / p_{1}^{i}$. Solving the latter yields

$$
s_{1}^{i}\left(y_{1}^{j}\right)= \begin{cases}N V^{i}-y_{1}^{j} & \text { when } y_{1}^{j} \leq N V^{i} \\ q_{1}^{i}\left(N V^{i}\right) & \text { otherwise }\end{cases}
$$

where $N V^{i} \equiv \bar{F}_{1}^{-1}\left(c_{1}^{i} / p_{1}^{i}\right)$ corresponds to the newsvendor stocking quantity of firm $i$ when it is a monopolist, i.e., when $y_{1}^{j}=0$.

In Figure 1 we plot $s_{1}^{i}\left(y_{1}^{j}\right)$ and $s_{1}^{j}\left(y_{1}^{i}\right)$. For simplicity, we omit the subindex $t=1$, and firm $i$ is such that $c^{i} / p^{i}<c^{j} / p^{j}$. The base-stock functions $s^{i}\left(y^{j}\right)$ and $s^{j}\left(y^{i}\right)$ intersect only once, which means that in the unconstrained competitive game there exists a unique Nash equilibrium, which we denote $E=\left(E^{i}, E^{j}\right)$. The shaded regions (I)-(IV) are used in the proofs provided in the technical appendix.

\footnotetext{
${ }^{7}$ In Cachon and Terwiesch (2005) these two models are defined in terms of the reactive capacity, and are referred to as limited and unlimited but expensive respectively.
} 
If now we allow the initial inventory levels to be non-zero (i.e., we consider the constrained problem), given the convexity of $r_{1}^{i}$, it follows that firm $i$ 's best response is

$$
b_{1}^{i}\left(x_{1}^{i}, y_{1}^{j}\right)=\max \left\{x_{1}^{i}, s_{1}^{i}\left(y_{1}^{j}\right)\right\} .
$$

Therefore, in the constrained competitive game, a graph of the best responses would look just as Figure 1, except that the vertical and horizonal stretches would move right and down respectively. Hence, a graphical argument is enough to prove our first result.

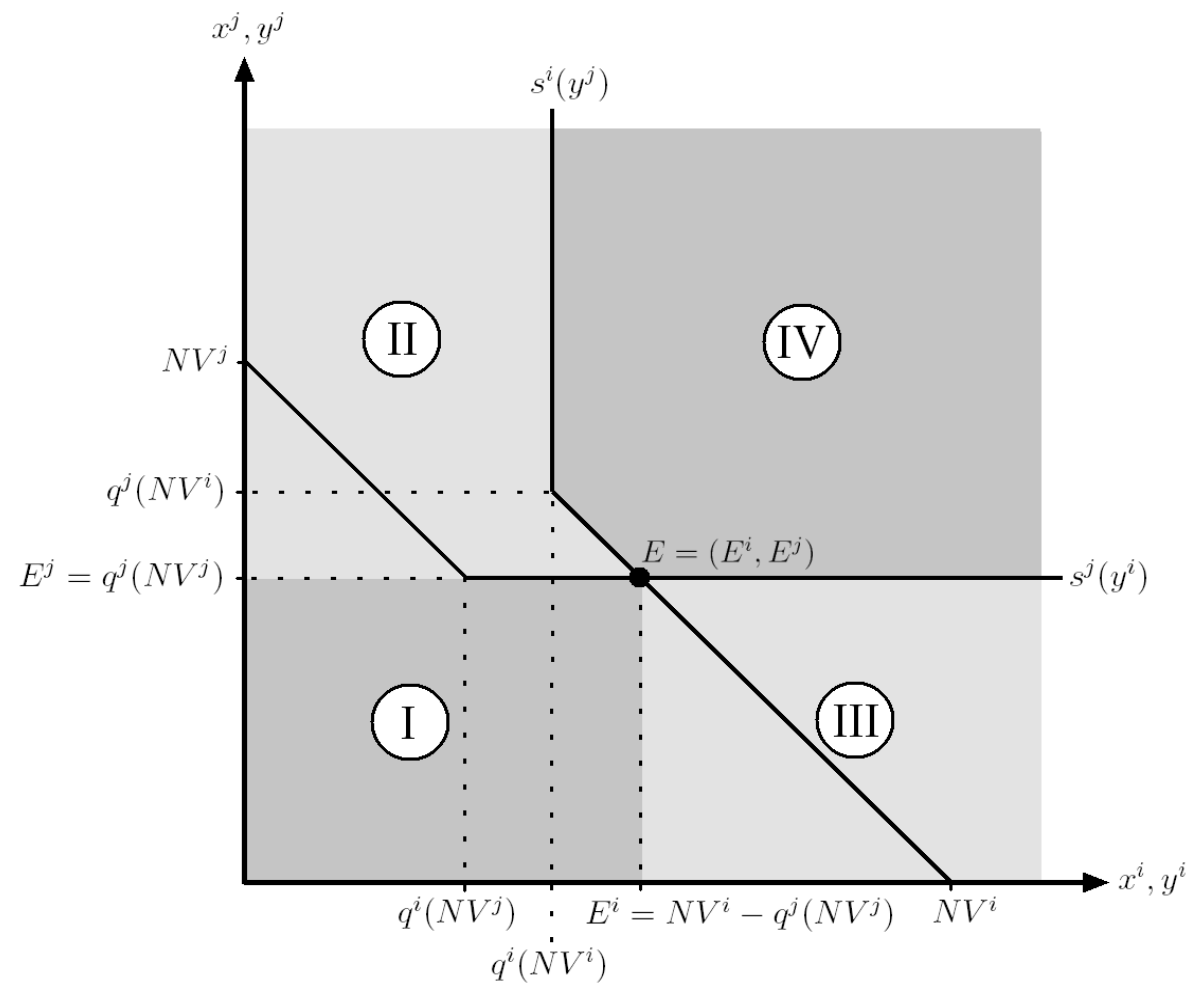

Figure 1: Unconstrained single-period base-stock functions.

Theorem 1 For all $\left(x_{1}^{1}, x_{1}^{2}\right)$, there exists a unique Nash equilibrium $\left(e_{1}^{1}\left(x_{1}^{1}, x_{1}^{2}\right), e_{1}^{2}\left(x_{1}^{2}, x_{1}^{1}\right)\right)$ of the stocking game. In addition, we can characterize $\left(e_{1}^{1}, e_{1}^{2}\right)$ as follows. Without loss of generality, assume that $\frac{c_{1}^{1}}{p_{1}^{1}} \leq \frac{c_{1}^{2}}{p_{1}^{2}}$ (firm 1 has a higher gross margin). Then $e_{1}^{2}\left(x_{1}^{2}, x_{1}^{1}\right)=\max \left\{x_{1}^{2}, q_{1}^{2}\left(N V^{2}\right)\right\} \quad$ and $e_{1}^{1}\left(x_{1}^{1}, x_{1}^{2}\right)=\max \left\{x_{1}^{1}, q_{1}^{1}\left(N V^{1}\right), N V^{1}-e_{1}^{2}\left(x_{1}^{2}, x_{1}^{1}\right)\right\}$.

We can see from Theorem 1 that the equilibrium strategy of the lower-margin firm is independent of the competitor's initial inventory level $x_{1}^{1}$. Indeed, when the initial inventory levels are zero, then the higher cost drives the lower-margin firm to ignore the overflow from 
the other company. The reverse is not true: the higher-margin equilibrium strategy may depend on $x_{1}^{2}$.

Theorem 3 of Lippman and McCardle (1997) proves that the unconstrained competitive game with symmetric retailers has a unique Nash equilibrium. The result requires the initial allocation functions to be deterministic and strictly increasing, just as in our setting. Theorem 1 in this paper extends the result by Lippman and McCardle in the sense that we consider the constrained game (i.e., the initial inventory levels can be non-zero) and we allow for asymmetric retailers (i.e., they can face different costs and prices).

From a technical standpoint, the unique equilibrium in the single-period problem follows from the fact that the unconstrained expected profit $r_{1}^{i}$ is concave in firm $i$ 's action $y_{1}^{i}$. In order to prove the existence of a unique pure-strategy subgame-perfect equilibrium in the two-period game, we will need to show that $r_{2}^{i}$ is (strictly) quasi-concave in $y_{2}^{i}$. For that, we first need to show that $\pi_{1}^{i}$, the equilibrium expected profit in the single-period problem, is concave in firm $i$ 's initial stock level $x_{1}^{i}$. Note that we have to consider the equilibrium expected profit because, by the definition of subgame-perfect, the retailers assume that in the next period a Nash equilibrium will be played, given any initial state $\left(x_{1}^{1}, x_{1}^{2}, I_{1}\right)$ (we refer the reader back to the discussion at the end of $\S 3.2$ ). The following proposition provides the theoretical result that we will need as a building block in the next section.

Proposition 1 For all $I_{1}$, the expected equilibrium profit $\pi_{1 \mid I_{1}}^{i}\left(x_{1}^{i}, x_{1}^{j}\right)$ is concave in $x_{1}^{i}$, for all $x_{1}^{j}$.

\subsection{Two-Period Case $(T=2)$}

We now look at the two-period case which is arguably the most important one since QR models typically only have two periods. In fact, the two models we consider seem to concentrate most of the attention in the literature (see, for instance, Cachon and Terwiesch 2005). Figure 2 shows a schematic description of both models.

\subsubsection{Demand Signal}

The first QR model we consider is based on the one studied (for a single firm) in Iyer and Bergen (1997). Similar sequence of events have been used in several other papers (see, for instance, Cachon and Swinney 2007). The planning horizon is divided in two periods. The last one represents the retail selling season, whereas the first one represents a period during which a demand signal is revealed. The latter could simply represent data that is collected right before the season starts (for example, in fashion shows, mock stores, focus groups, or 

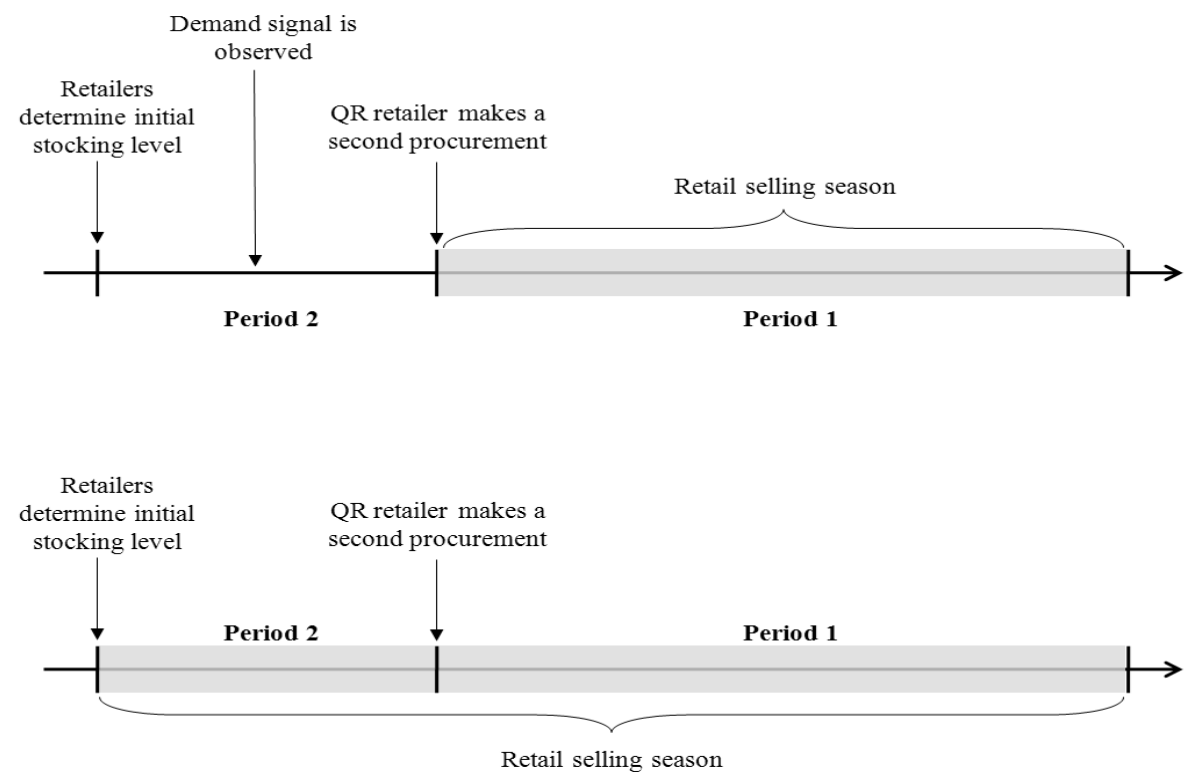

Figure 2: QR models, demand signal (top) and mid-season replenishment (bottom).

by consulting experts). We assume that the demand signal is informative, meaning that it is correlated with the actual demand during the season. Otherwise, this model reduces to the single-period problem studied in the previous section. A QR retailer can place orders before and after observing the demand signal, whereas a traditional retailer, due to longer lead times, can only place a single order before the additional demand information becomes available. The sequence of events for a QR retailer are depicted in Figure 2 (top timeline).

Given that there is no demand realization in the initial period $\left(R_{2}^{i}=R_{2}^{j}=0\right)$, the unconstrained expected profit $r_{2}^{i}$ reduces to

$$
r_{2}^{i}\left(y_{2}^{i}, y_{2}^{j}\right)=-c_{2}^{i} y_{2}^{i}+\mathbb{E}\left\{\pi_{1 \mid I_{1}}^{i}\left(y_{2}^{i}, y_{2}^{j}\right)\right\}
$$

where the expectation is with respect to the (a priori) distribution of $I_{1}$. Note that $r_{2}^{i}\left(y_{2}^{i}, y_{2}^{j}\right)$ is concave in $y_{2}^{i}$, for any $y_{2}^{j}$ (from Proposition 1 ). Therefore, the existence of a pure-strategy (sub-game perfect) Nash equilibrium in the unconstrained competitive game is guaranteed by Theorem 1.2 in Fudenberg and Tirole (1991). ${ }^{8}$ Moreover, also due to the concavity of $r_{2}^{i}$, firm $i$ 's best response is a base-stock policy $s_{2}^{i}\left(y_{2}^{i}\right)$. Therefore, just as in the singleperiod model, the best-response function in the constrained game is equal to $b_{2}^{i}\left(x_{2}^{i}, y_{2}^{i}\right)=$ $\operatorname{argmax}_{y_{2}^{i} \geq x_{2}^{i}}\left\{r_{2}^{i}\left(y_{2}^{i}, y_{2}^{j}\right)\right\}=\max \left\{x_{2}^{i}, s_{2}^{i}\left(y_{2}^{i}\right)\right\}$. The following Theorem provides conditions

\footnotetext{
${ }^{8}$ The theorem actually requires that the strategy space is compact. Since the retailers would never order an infinite stock, it is always possible to restrict their actions to a compact set.
} 
under which the equilibrium is unique. ${ }^{9}$ The proof is omitted since it follows directly from Proposition 1 and the derivatives computed in Table 3. After stating and discussing the theorem we provide an example that shows an application of this result.

Theorem 2 If $c_{2}^{i} \neq c_{1}^{i}$ for $i=1,2$, then the stocking game with a demand signal has a unique subgame-perfect equilibrium. In that equilibrium, both retailers play pure-strategies.

Three technical observations about Theorem 2 are worth noting. First, when $c_{2}^{i}=c_{1}^{i}$, the equilibrium exists (in fact, not ordering in the initial period would be an equilibrium), but in general it might not be unique (though the profits achieved are the same under any equilibrium). ${ }^{10}$ Second, the theorem rules out the existence of a mixed-strategy equilibrium, since in the unique equilibrium the retailers play pure-strategies. Third, per Assumption (A4), the strategies played by the retailers must be Markovian. Hence, what we actually prove is that there exists a unique Markovian perfect equilibrium (see $\S 13$ in Fudenberg and Tirole 1991). However, since the state $\left(x_{t}^{1}, x_{t}^{2}, I_{t}\right)$ contains all the relevant information from the past (i.e., it is sufficient), it is not hard to see that any strategy that induces a sub-game perfect equilibrium must be Markovian, or at least equivalent to a Markovian strategy. ${ }^{11}$ This observation justifies the claim that there is a unique subgame-perfect equilibrium. A similar justification is given in Hall and Porteus (2000).

Example 1 (Iyer and Bergen 1997): The two-period demand signal case allows us to model the competition between a traditional retailer (i.e., one with very long lead times) and a QR retailer that is modeled as in Iyer and Bergen (1997). In that paper, demand during the retail selling season is assumed to be normally distributed, i.e. $D_{1} \sim N\left(\theta, \sigma^{2}\right)$. The variance $\sigma^{2}$ is assumed to be known, whereas the average size of the market $\theta$ is uncertain. Information about $\theta$ in the initial period is modeled as a normal distribution with mean $\mu$ and variance $\tau^{2}$. Thus, at time $t=2$, the prediction of season demand is normally distributed with mean $\mu$ and variance $\sigma^{2}+\tau^{2}$. Then the demand signal $d$ is realized, and the QR retailer performs a Bayesian update of its belief regarding $\theta$. In other words, we have that $I_{1}=\{d\}$, and $D_{1 \mid I_{1}} \sim N\left(\mu(d), \sigma^{2}+1 / \rho\right)$, where

$$
\mu(d)=\frac{\sigma^{2} \mu+\tau^{2} d}{\sigma^{2}+\tau^{2}} \quad \text { and } \quad \rho=\frac{1}{\sigma^{2}}+\frac{1}{\tau^{2}}
$$

\footnotetext{
${ }^{9}$ When the equilibrium is not unique, $s_{2}^{i}\left(y_{2}^{i}\right)$ and $b_{2}^{i}\left(x_{2}^{i}, y_{2}^{i}\right)$ are actually correspondences rather than functions.

${ }^{10}$ Notice that, if $c_{2}^{i}>c_{1}^{i}$, then the equilibrium is to not order anything in the initial period. On the contrary, if $c_{2}^{i}<c_{1}^{i}$, then a positive amount is ordered in $t=2$.

${ }^{11}$ To be precise, there is a Markovian strategy that plays the exact same actions than the non-Markovian one, for all possible histories.
} 
Note that $1 / \rho<\tau^{2}$. Therefore, after the realization of the demand signal $d$ is observed, the $\mathrm{QR}$ retailer has a more accurate prediction of season demand. The traditional retailer cannot make use of the demand signal because of long lead times. In other words, it cannot place a second order after the demand signal is realized. This is incorporated in our model by letting $c_{1}^{j}=p_{1}^{j}$ (here we assume that index $j$ represents the traditional retailer). As long as it is cheaper to order in the initial period (for both retailers), the conditions of Theorem 2 hold, and the competition between the QR response and the traditional retailer has a unique subgame-perfect equilibrium.

\subsubsection{Mid-Season Replenishment}

In the previous QR model, the demand signal does not deplete stock. That is, the acquisition of additional demand information in the initial period does not affect the inventory that is carried over to the final period. This simplifies the analysis and allows us to prove the existence of a unique equilibrium under fairly general conditions (see Table 1). In the current section, we consider a second QR model that differs from the previous one in few subtle but fundamental aspects. Specifically, the selling season comprises both periods, the actual sales that occur in the initial period play the role that the demand signal previously had, and the procurement in the final period comes to replenish the inventory that has been depleted (hence the name for this case). The respective sequence of events is depicted in the bottom of Figure 2). An example that would fit this QR model is the Sport Obermeyer case (Fisher and Raman 1996), where $20 \%$ of initial sales provides an excellent estimation of the remaining $80 \%$.

As before, the unconstrained expected profit $r_{2}^{i}\left(y_{2}^{i}, y_{2}^{j}\right)$ in the initial period $(t=2)$ is given by Equation (5). However, now the profit-to-go $\pi_{1 \mid I_{1}}^{i}$ is evaluated in the remaining inventory, and $r_{2}^{i}$ is no longer guaranteed to be concave. Fortunately, we are able to show that it is quasi-concave under certain conditions to be introduced next, and hence, the optimal policy is still a base-stock policy dependent on $y_{2}^{j}$.

\section{Proposition 2 Assume that}

(i) for $i=1,2, c_{1}^{i} \geq c_{2}^{i}$ and $p_{1}^{i} \leq p_{2}^{i}$;

(ii) $D_{2}$ has infinite support and a log-concave p.d.f., i.e., $\log \left(f_{2}(d)\right)$ is concave in $d$;

(iii) $I_{1}=\left\{D_{2}\right\}$ and $D_{1 \mid I_{1}}=k D_{2}+\epsilon$, where $k \geq 0$ and $\epsilon$ is a random variable independent of $D_{2}$ with p.d.f. $g$, such that, for all $x$, for $i=1,2$, 


$$
\max \left\{0, \frac{f_{2}^{\prime}}{f_{2}}\left(\left(q_{2}^{i}\right)^{-1}(x)\right)\right\} \leq \max \left\{0, \frac{g^{\prime}}{g}\left(\left(q_{1}^{i}\right)^{-1}(x)\right)\right\}
$$

(iv) if $k>0$, then $q_{1}^{i}(d)=\alpha_{1}^{i} d$ for $i=1,2$.

Then, $r_{2}^{i}\left(y_{2}^{i}, y_{2}^{j}\right)$ is quasi-concave for all $y_{2}^{j}$, and the constrained best response is $b_{2}^{i}\left(x_{2}^{i}, y_{2}^{j}\right)=$ $\max \left\{x_{2}^{i}, s_{2}^{i}\left(y_{2}^{j}\right)\right\}$, where $s_{2}^{i}\left(y_{2}^{j}\right)$ is the (unconstrained) base-stock level, which is unique.

The first condition $(i)$ in Proposition 2 requires that the margins do not increase over time. This would be the case if the price is fixed and the mid-season replenishment is more expensive than the initial procurement. The second condition (ii) requires demand $D_{2}$ to be log-concave with an infinite support. ${ }^{12}$ The former is needed to generate bounds in the proof, and the latter is used to guarantee a unique maximum. The third condition (iii) specifies the dependency between $D_{1}$ and $D_{2}$ that is allowed. Notice that the inequality (9) is satisfied if $f_{2}$ has a decreasing p.d.f., or if it is not larger than $g$ in the likelihood ratio order. The latter is typically the case when the initial period represents less than half of the total season. Finally, the last condition ( $i v$ ) requires (for technical reasons) a linear splitting rule in the final period whenever $D_{1}$ and $D_{2}$ are not independent.

The proof of Proposition 2 involves several claims that are stated (and proved) in the technical appendix, but the central idea is to show that the following inequality holds

$$
\frac{\partial^{2} r_{2}^{i}}{\left(\partial y_{2}^{i}\right)^{2}}<\phi_{2}\left(\left(q_{2}^{i}\right)^{-1}\left(y_{2}^{i}\right)\right)\left(\frac{\partial r_{2}^{i}}{\partial y_{2}^{i}}\right), \text { where } \phi_{2}(y)=\max \left\{0, \frac{f_{2}^{\prime}}{f_{2}}(y)\right\} .
$$

Note that quasi-concavity follows directly from (10), since any critical point is a maximum, and therefore, the function $r_{2}^{i}$ is unimodal. We can now state the main result of this section.

Theorem 3 If the conditions (i)-(iv) of Proposition 2 are satisfied, then the stocking game with mid-season replenishment has a unique pure-strategy subgame-perfect equilibrium.

As in Theorem 2 for the demand signal case, Theorem 3 shows existence and uniqueness of a pure-strategy subgame-perfect equilibrium. However, there are a some differences. First, Theorem 3 requires a few more conditions than Theorem 2 (see Table 1 for a comparison). Second, since in the mid-season replenishment case $r_{2}^{i}$ is quasi-concave rather than concave, we cannot rule out the existence of a mixed equilibrium. Another consequence is that the equilibrium profit $\pi_{2}^{i}$ defined in Equation (7) is not concave either, and therefore, we are not able to extend Theorem 3 to a larger number of periods.

\footnotetext{
${ }^{12}$ To be precise, the support must be either the real line or an interval of the type $[a,+\infty)$.
} 
Despite the additional conditions required in Theorem 3, there are several interesting cases for which they hold. Two of them are given in the next corollaries. Corollary 1 shows the simplest application of Theorem 3 by assuming i.i.d. demand. On contrary, Corollary 2 shows an application with demand that is correlated across periods. We then use the latter in an example that resembles the QR model in the Sport Obermeyer case (Fisher and Raman 1996).

Corollary 1 (Independent demands) Assume that $c_{1}^{i} \geq c_{2}^{i}, p_{1}^{i} \leq p_{2}^{i}$, and $q_{1}^{i}=q_{2}^{i}$ for $i=1,2$. If the demands $D_{2}, D_{1}$ are i.i.d. and $D_{2}$ is log-concave with infinite support, then the stocking game with mid-season replenishment has a unique pure-strategy subgame-perfect equilibrium.

Corollary 2 (Correlated demands) Assume that $c_{1}^{i} \geq c_{2}^{i}, p_{1}^{i} \leq p_{2}^{i}$, and $q_{1}^{i}(d)=q_{2}^{i}(d)=$ $\alpha^{i} d$, for $i=1,2$. Let $\epsilon_{2}, \epsilon_{1}$ be two independent random variables such that $D_{2}=\epsilon_{2}$ and $D_{1 \mid I_{1}}=k D_{2}+\epsilon_{1}$, with $k>0$ (thus, $\left.\rho \equiv \operatorname{Corr}\left(D_{2}, D_{1}\right)=k \sqrt{\operatorname{Var}\left(D_{2}\right) / \operatorname{Var}\left(D_{1}\right)}>0\right)$. Furthermore, let either

- $\epsilon_{2}, \epsilon_{1}$ follow normal distributions with parameters $\left(\mu_{2}, \sigma_{2}\right)$ and $\left(\mu_{1}, \sigma_{1}\right)$ respectively and $\mu_{2} \leq \mu_{1}$ and $\sigma_{2} \geq \sigma_{1} ;$ or

- $\epsilon_{2}, \epsilon_{1}$ follow truncated normal distributions with parameters $\left(\mu_{2}, \sigma_{2}\right)$ and $\left(\mu_{1}, \sigma_{1}\right)$ respectively and $\mu_{2} \leq \mu_{1}$ and $\frac{\sigma_{2}}{\mu_{2}} \geq \frac{\sigma_{1}}{\mu_{1}}$; or

- $\epsilon_{2}, \epsilon_{1}$ follow gamma distributions with parameters $\left(a_{2}, \theta_{2}\right)$ and $\left(a_{1}, \theta_{1}\right)$ respectively and $\theta_{2} \leq \theta_{1}$ and $1 \leq a_{2} \leq a_{1}$; or

- $\epsilon_{2}, \epsilon_{1}$ follow exponential distributions.

In the four cases above, the stocking game with mid-season replenishment has a unique pure-strategy subgame-perfect equilibrium.

Example 2 (Fisher and Raman 1996): Consider the case when the demand vector $\left(D_{2}, D_{1}\right)$ follows a multivariate normal with marginal distributions $D_{i} \sim N\left(\mu_{i}, \sigma_{i}\right)$ and covariance $\operatorname{Cov}\left(D_{2}, D_{1}\right)=\rho \sigma_{2} \sigma_{1}$, with $\rho \geq 0$. Then we have that, the demand in the last period conditional on the demand realization in the initial period, is given by $D_{1 \mid I_{1}}=k D_{2}+\epsilon_{1}$ with $k=\rho \sigma_{1} / \sigma_{2}$, and $\epsilon_{1}$ is normally distributed with parameters $\left(\mu_{1}-k \mu_{2}, \sigma_{1} \sqrt{1-\rho^{2}}\right)$ and is independent of $D_{2}\left(=\epsilon_{2}\right)$. Notice that, if there is positive correlation $(\rho>0)$, then $\operatorname{Var}\left(D_{1 \mid I_{1}}\right)=\sigma_{1}^{2}\left(1-\rho^{2}\right)<\sigma_{1}^{2}=\operatorname{Var}\left(D_{1}\right)$. In other words, as in Example 1, the updated 
forecast $D_{1 \mid I_{1}}$ is more accurate than the unconditional prediction of $D_{1} \cdot{ }^{13}$ It can be shown that the condition on the normal distributions in Corollary 2, in this case, reduces to

$$
\mu_{2}<\mu_{1} \quad \text { and } \quad \sigma_{2} \geq \sigma_{1} \max \left\{\sqrt{1-\rho^{2}}, \rho \frac{\mu_{2}}{\mu_{1}-\mu_{2}}\right\} \text {. }
$$

Hence, if the latter holds, then Theorem 3 applies. In particular, this would be the case if a QR retailer can place an order after observing a small fraction (e.g., 20\%) of the total season demand (so that $\mu_{2} \ll \mu_{1}$ ), and these early sales provide relevant information about what should be expected in the remainder of the season (i.e., the correlation $\rho$ is high).

\subsection{Two or More Periods $(T \geq 2)$}

Most QR models in the literature only consider two periods, which also represents the actual situation of a large number of retailers in practice, who have at most two procurement opportunities in a given season. That justifies dedicating an entire section to study the two-period case (c.f. §4.2). Now we turn to the general case with two or more periods, which again is partly motivated by fast fashion retailers like Zara who have the ability to replenish stock several times during the selling season. However, as it was mentioned before, we found that extending Theorem 3 to more than two periods was not directly feasible with the methodology used to prove Proposition 2. ${ }^{14}$ Indeed, the equilibrium profit $\pi_{t}^{i}\left(y_{t}^{i}, y_{t}^{j}\right)$ may not be concave nor quasi-concave in $y_{t}^{i}$ for $t \geq 2$. This can be worked around if we can prove that the competitor's best response $b_{t}^{j}$ is constant within a certain range. In this section we show two cases when that is possible. Namely, when one of the retailers does not act strategically, and when they are symmetric.

\subsubsection{Inventory control when one retailer is passive}

We consider here the special situation where one retailer is passive and cannot place orders during the season: we assume that retailer 2 is such that $\min _{t}\left\{c_{t}^{2}\right\} \geq \max _{t}\left\{p_{t}^{2}\right\}$ for all $t$, i.e., it is too expensive to acquire inventory and thus $b_{t}^{2}\left(x_{t}^{2}, y_{t}^{1}\right)=x_{t}^{2}$. In other words, competition here does not really take place as a game, but instead retailer 1 simply responds to the inventory level of retailer 2, who is passive and cannot purchase inventory besides its initial quantity $x_{T}^{2}$. This situation models the existing competition between a fashion-leader, i.e., a retailer that designs and produces early, without even considering the competition, and a fashion-follower, i.e., a retailer that "copies" the fashion-leader's designs and is able to

\footnotetext{
${ }^{13}$ Under the conditions of Proposition 2, it always holds that $\operatorname{Var}\left(D_{1}\right)=k^{2} \operatorname{Var}\left(D_{2}\right)+\operatorname{Var}(\epsilon) \geq \operatorname{Var}(\epsilon)=$ $\operatorname{Var}\left(D_{1 \mid I_{1}}\right)$.

${ }^{14}$ The demand signal case can be extended to have an arbitrary number of periods before the season begins, but essentially it reduces to the two-period case.
} 
produce the items quickly through QR. This could represent the case of Zara, "a very quick fashion follower", according to Ghemawat and Nueno (2003), p.12.

Theorem 4 Assume that

(i) $c_{T}^{1} \leq \ldots \leq c_{1}^{1}$ and $p_{T}^{1} \geq \ldots \geq p_{1}^{1}$, and $\min _{t}\left\{c_{t}^{2}\right\} \geq \max _{t}\left\{p_{t}^{2}\right\}$;

(ii) demand is independent across periods, and for $t=T, \ldots, 2, D_{t}$ is log-concave and has infinite support;

(iii) for all $d$,

$$
\frac{f_{t}^{\prime}}{f_{t}}\left(\left(q_{t}^{1}\right)^{-1}(d)\right) \leq \max \left\{0, \frac{f_{t-1}^{\prime}}{f_{t-1}}\left(\left(q_{t-1}^{1}\right)^{-1}(d)\right)\right\}
$$

Then, the stocking game has a unique pure-strategy subgame-perfect equilibrium where, in each period, firm 1 follows a base-stock policy with order-up-to level $s_{t}^{1}\left(x_{t}^{2}\right)$, and firm 2 never orders.

When a retailer cannot order during the season and starts with an exogenously given inventory level, its competitor faces a multi-period inventory problem. The theorem shows that this non-trivial problem (as current inventory decisions influence future demands) is well-behaved and that a base-stock policy optimal. Of course, the base-stock level depends on the competitor's inventory level.

\subsubsection{Symmetric retailers}

When additional conditions are placed on the retailers and the demand structure, stronger results can be derived. This section provides a general result for an arbitrary number of periods, when the retailers are symmetric, i.e., both retailers have identical costs and prices in every period. Notice that our definition of symmetry still allows the retailers to a have different initial inventories. Here, the generalization of the model to $T \geq 2$ can be obtained with standard methods, assuming that the demand splitting rule is linear in all periods.

Theorem 5 Assume that, given $T \geq 1$, for all $t=T, \ldots, 1$, costs and prices are identical for both firms, i.e., $c_{t}^{1}=c_{t}^{2}$ and $p_{t}^{1}=p_{t}^{2}$. In addition, assume that $q_{t}^{i}\left(D_{t \mid I_{t}}\right)=\alpha^{i} D_{t \mid I_{t}}$ for all $t=T, \ldots, 1$.

Then, the T-period stocking game has a unique pure-strategy subgame-perfect equilibrium, for all $I_{T}$. In this equilibrium, at period $t, e_{t \mid I_{t}}$ is characterized by

$$
e_{t \mid I_{t}}^{i}\left(x^{i}, x^{j}\right)=\max \left\{x^{i}, \alpha^{i} s_{t \mid I_{t}}\right\},
$$


where $s_{t \mid I_{t}}$ is the monopoly base-stock level, i.e., the solution to the inventory problem for a single firm that receives $D_{t \mid I_{t}}$ per period.

As stated in the theorem, the equilibrium level of retailer $i$ does only depend on its own inventory level $x^{i}$ and is independent of the competitor's inventory level $x^{j}$. To be more precise, we show that retailer $i$ has a dominant strategy that involves the monopoly stocking quantity $s_{t \mid I_{t}}$, defined as follows.

Consider the following dynamic program, with $c_{t}=c_{t}^{1}=c_{t}^{2}$ and $p_{t}=p_{t}^{1}=p_{t}^{2}$ :

$$
\begin{aligned}
& U_{0 \mid I_{0}}(x) \equiv 0 \\
& U_{t \mid I_{t}}(x)=c_{t} x+\max _{y \geq x}\left\{-c_{t} y+p_{t} \mathbb{E} \min \left\{y, D_{t \mid I_{t}}\right\}+\mathbb{E} U_{t-1 \mid I_{t-1}}\left(\left(y-D_{t \mid I_{t}}\right)^{+}\right)\right\}
\end{aligned}
$$

Since this is a standard inventory problem, it is easy to see that the optimal policy for $t$ is to set $y=\max \left\{x, s_{t \mid I_{t}}\right\}$, where $s_{t \mid I_{t}}$ is the order-up-to level of the respective unconstrained problem. This is the quantity used in Theorem 5 .

The proof methodology is relatively standard, and uses the same lines of Lippman and McCardle (1997). Essentially, we show by induction that $r_{t \mid I_{t}}^{i}\left(y^{i}, y^{j}\right)$ is concave in $y^{i}$ (for all $y^{j}$ ) and submodular, which yields that the optimal replenishment policy is a base-stock policy. Also, a key part of the proof involves that when $\frac{y^{i}}{\alpha^{i}} \leq \frac{y^{j}}{\alpha^{j}}$, retailer $i$ never receives overflows from $j$, in period $t$ or in future periods $t-1, \ldots, 1$. As a result, the best response $b_{t \mid I_{t}}^{i}$ is independent of $y^{j}$, and equal to $\alpha^{i} s_{t \mid I_{t}}$, which yields the equilibrium structure presented in Theorem 5. Figure 3 illustrates the shape of the best-response functions described by the theorem. This plot is for $T=2, c_{t}^{i}=0.6, p_{t}^{i}=1, D_{t}$ uniform $[0,1]$, for $t=2,1$ and $i=1,2$, $x_{2}^{1}=x_{2}^{2}=0$ and $\alpha^{1}=30 \%$. We observe that, indeed, $b_{2}^{i}$ is flat in $y_{2}^{j}$, when $\frac{y_{2}^{j}}{\alpha^{j}} \geq s_{t \mid I_{t}}$.

We thus completely characterize the multi-period equilibrium in the case of symmetric firms (in terms of cost and price, not necessarily in terms of market share). Note that it is allowed that the firms start with any initial inventory. This contrasts with the work by Avsar and Baykal-Gürsoy (2002), who require that the initial inventory levels of retailers are below their equilibrium stationary base-stock levels. Also note that it is still possible that spill-overs between retailers occur, even though the equilibrium quantity of each retailer is independent of the competitor's inventory level, see Equation (12).

Notice that, when both firms start with zero inventory at $t=T$, the aggregate industry inventory is equal to $\alpha^{1} s_{T \mid I_{T}}+\alpha^{2} s_{T \mid I_{T}}=s_{T \mid I_{T}}$, which is also the industry inventory target level under a monopoly, i.e., when firms 1 and 2 merge. Our result thus extends Theorem 


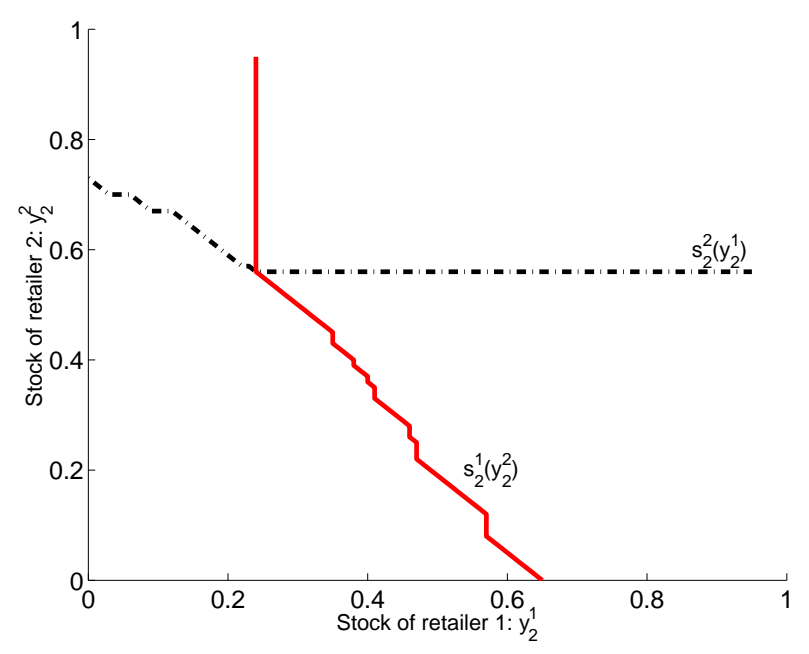

Figure 3: Shape of the best-response functions $b_{2}^{i}$ in the case of symmetric firms.

3 of Lippman and McCardle (1997) to a multi-period setting with demand learning. Unfortunately, when costs are asymmetric, it turns out that the best response $b_{t \mid I_{t}}^{i}$ is never independent of $y^{j}$, which prevents the use of some of the arguments of the proof. Interestingly, the same sort of property is used in Nagarajan and Rajagopalan (2005) by requiring that the newsboy ratio is larger than 0.5 .

\section{Quick vs. Slow Response Competition}

In this section, we provide an extensive numerical study on the two-period $(T=2)$ inventory game. While simple, the two-period case captures most of the interesting dynamics arising as one moves from a single-period game to a multi-period one. In addition, Theorems 2 and 3 provide the theoretical results that guarantee the existence and uniqueness of equilibrium. In this section we compute such equilibrium and perform comparative statics, i.e. we study how the equilibrium depends on the key parameters of the model. We do this numerically since analytical comparative statics in our model are not straightforward.

We are interested in quantifying the advantages of a QR retailer over a SR (slow response) competitor, and compare inventory levels and profits of such competitive situation when compared to the "traditional" competition between two SR retailers (we assume that both start with zero inventory). For this purpose, we consider three main situations, all falling within our generic model presented before (c.f. §3). The first case, that we take as a benchmark, considers two SR retailers, in the sense that they can only place orders before the start of the selling season, at a cost $c^{S R}$. Thus, they are not able to place an order at time $t=1$. In this situation, the competition corresponds to the single period equilibrium 


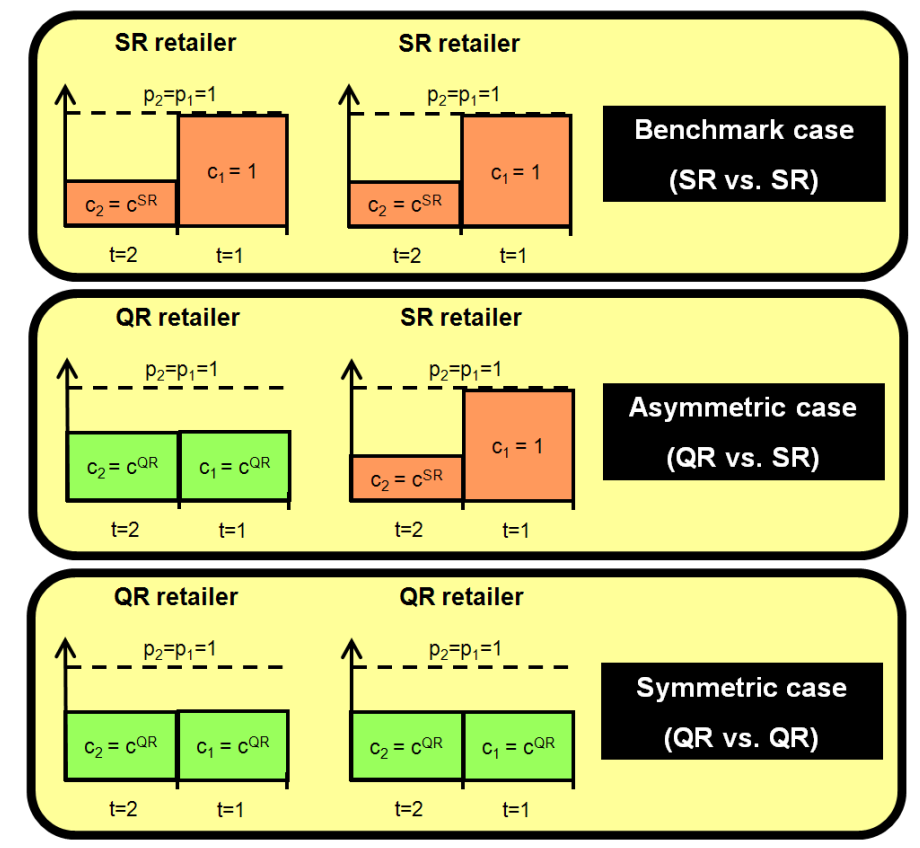

Figure 4: Three market configurations: SR vs. SR competition (top), QR vs. SR competition (middle) and QR vs. QR competition (bottom).

identified in Lippman and McCardle (1997). The second case, which is the focus of the paper, considers the asymmetric competition between a SR and a QR retailer. The SR retailer is identical to the ones of the first case. On the contrary, the fast retailer is characterized by the possibility to place orders in both periods, at a cost $c^{Q R}$. We allow it to have the same or possibly higher ordering cost than the slow retailer (i.e., $c^{Q R} \geq c^{S R}$ ), as in reality. Finally, the third case considers two symmetric QR retailers, which will be used to evaluate how much of the advantage of being $\mathrm{QR}$ arises from the asymmetries between retailers. In our simulations, we use identical prices in each period $p_{1}^{i}=p_{2}^{i}=1$, for $i=1,2$. Therefore, if firm $i$ is a SR retailer, then $c_{2}^{i}=c^{S R}$ and $c_{1}^{i}=p_{1}^{i}=1$, whereas if firm $i$ is a QR retailer, then $c_{2}^{i}=c_{1}^{i}=c^{Q R}$. Figure 4 summarizes the three cases that we analyze throughout our numerical study.

Our demand model follows the structure presented in Proposition 2, namely, $D_{1 \mid I_{1}}=$ $k D_{2}+\epsilon$. This allows retailers to learn from the realization of $D_{2}$ and improve the forecast of the last period demand $D_{1}$. In the demand signal case, $D_{2}$ is the additional market information gained in the initial period, whereas in the mid-season replenishment case, $D_{2}$ is the actual demand in $t=2$. The parameter $k$ determines the correlation between $D_{2}$ and $D_{1}$. Specifically,

$$
\rho=\operatorname{Corr}\left(D_{2}, D_{1}\right)=\frac{k}{\sqrt{k^{2}+1}}
$$


Note that, in the figures below, we plot our results directly as a function of the demand correlation instead of $k$. For simplicity, and to avoid negative demand, we consider that $D_{2}$ and $\epsilon$ are identically distributed, and follow gamma distributions with mean $\mu=1$ and standard deviation $\sigma$. Finally, we use a linear splitting rule $q_{1}^{i}(d)=q_{2}^{i}(d)=\alpha^{i} d$ with a $50 \%$ market share for each retailer (i.e., $\alpha^{i}=\alpha=0.5$, for $i=1,2$ ).

\subsection{Equilibrium Inventory and Spill-Overs}

We start by comparing the (initial) equilibrium inventory levels between the benchmark (SR vs. SR) and the asymmetric (QR vs. SR) scenarios, when both retailers start with zero inventory, i.e., $x_{2}^{1}=x_{2}^{2}=0$. Of course, when both firms are SR retailers, all the inventory is ordered at the beginning of the season at $t=2$. In the $\mathrm{QR}$ vs. SR case, however, the $\mathrm{QR}$ retailer may place an additional order at $t=1$. Notice that in this section we solely look at the mid-season replenishment case. We do not consider here the demand signal case because, since $c_{2}^{i}=c_{1}^{i}$, the equilibrium in that game might not be unique (see the discussion after Theorem 2).

In Figure 5 we show the best-response function of each retailer in the benchmark (left) and in asymmetric case (right), when there is no demand correlation across periods. We observe, first, that the SR vs. SR case corresponds exactly to the single-period model. The QR vs. SR case figure illustrates a qualitatively different behavior: interestingly, the bestresponse function of the QR retailer is not necessarily decreasing in the inventory level of the competitor, as is always the case in single-period models. We found this behavior in most of the simulations performed. Furthermore we observe that the shape of both retailers's best-response functions is shifted down in the asymmetric case compared to the benchmark. Another interesting observation is that the best-response of the QR retailer is decreasing, then increasing, and eventually becomes constant. The latter is what should be expected. In fact, if the SR retailer has an extremely large quantity of inventory, then the best-response of the $\mathrm{QR}$ retailer is to ignore the competitor since it will not face spill-over demand. The same occurs when the competitor is passive and has a large amount of initial stock (c.f. §4.3.1).

Next, in Figure 6 we study how the equilibrium inventory levels in the initial period change when correlation is introduced and the cost of the QR retailer $\left(c^{Q R}\right)$ increases. The figure shows the equilibrium values for two situations: i.i.d. demand (and hence no forecast updating), and correlated demand with $\rho=0.7$ (in both situation $\sigma=0.3$ ). Notice that the values have been normalized by the total expected original demand $\left(\alpha \mathbb{E}\left\{D_{2}+D_{1}\right\}\right)$.

We depict the SR vs. SR equilibrium levels (diamond and star in the right bottom), and 

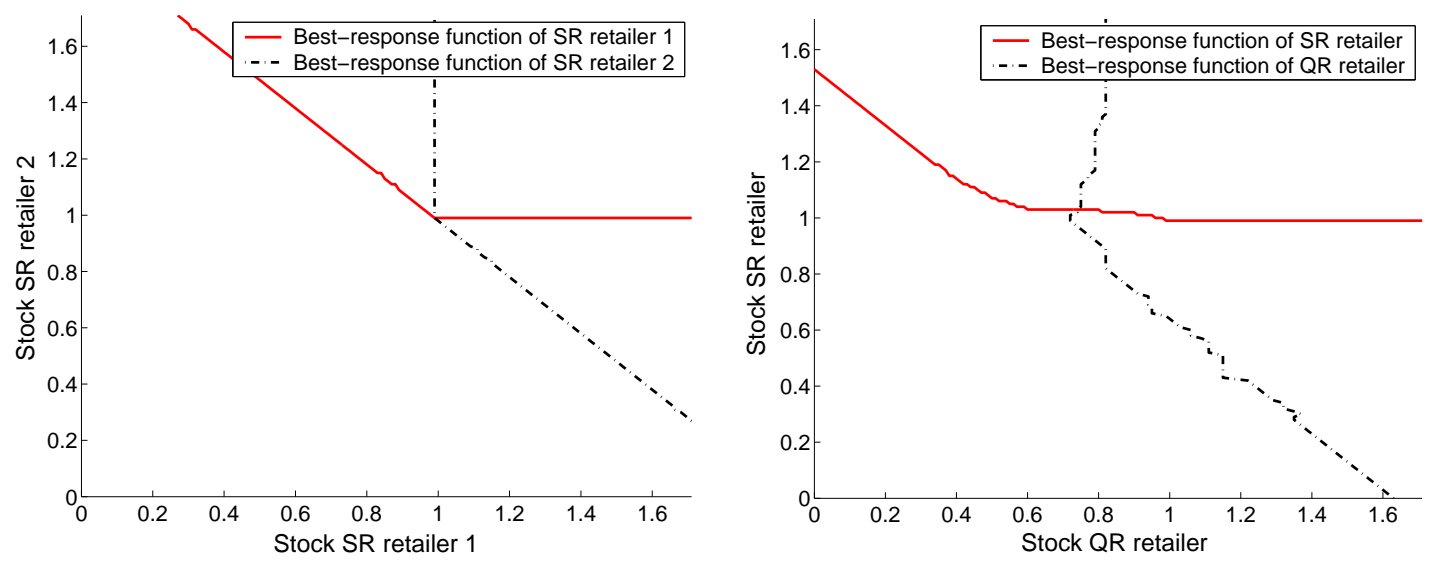

Figure 5: Best-response functions at $t=2$ for the benchmark case (left) and the asymmetric case (right). Here we use $k=0, \sigma=0.3$ and $c^{Q R}=c^{S R}=0.5$.

the curves of the QR vs. SR equilibrium levels for a variety of parameter values $c^{Q R}$ from 0.5 (bottom part of the curves) to 0.9 (top part of the curves). The cost $c^{S R}$ of the SR retailer(s) remains fixed at 0.5. First, we observe that the total initial inventory placed in the asymmetric case (QR vs. SR) decreases with respect to the benchmark case (SR vs. SR), and the decrease is more pronounced when demand is correlated and/or when the ordering cost of the QR retailer is higher. Intuitively, the industry initial inventory, i.e., the sum of both retailers' inventory, should be lower than in the SR vs. SR case because the QR retailer can postpone (part of) its order, and will do even more so when the value of performing forecast updates is higher (i.e., when $\rho$ is larger). Second, we observe that not only the inventory placed by the QR retailer is always lower than in the benchmark case, but the inventory level of the SR retailer is higher, even though the total industry inventory level decreases. Thus, the SR retailer takes advantage of competing with a QR retailer (which carries lower inventory) by placing higher inventory levels, and hence in the initial period captures higher sales in expectation.

Finally, to conclude this section, we investigate the magnitude of spill-overs between a $\mathrm{QR}$ and a SR retailer. As pointed out before, the SR retailer benefits from the decrease in inventory of the $\mathrm{QR}$ retailer and receives a spill-over in the initial period, $t=2$. However, as Figure 7 illustrates, the spill-over in the final period, $t=1$ goes from the SR retailer to the QR retailer and is significantly larger. For higher demand variability and demand correlation, the average spill-over may be as high as $10 \%$ of total demand sales. It is worth noting that in the demand signal case, since the demand realization (and hence, competition) only takes place in the last period, we generally observed considerably lower spill-overs. 


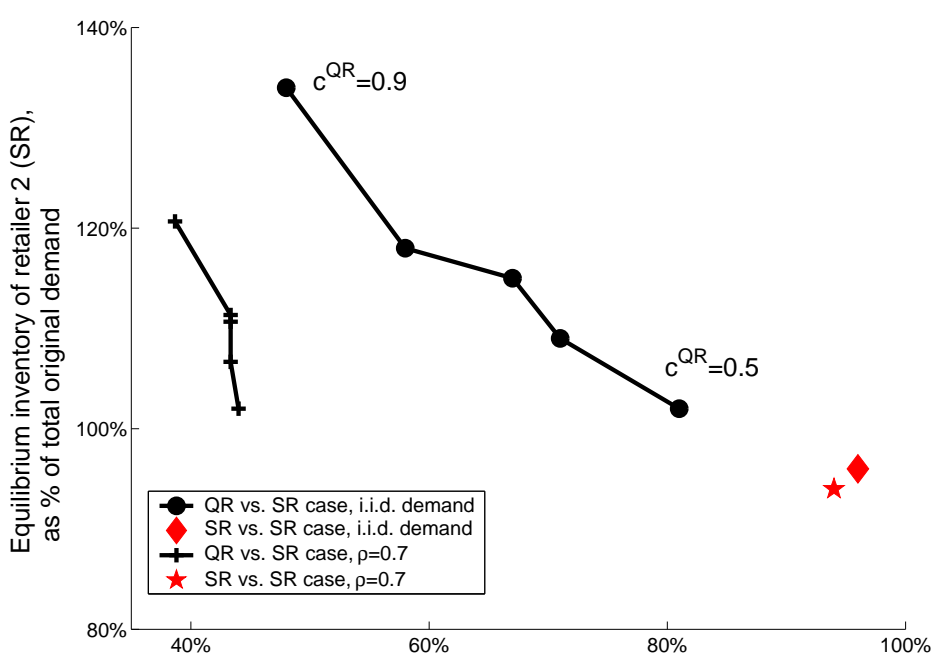

Equilibrium inventory of retailer 1 (QR or SR), as \% of total original demand

Figure 6: Initial equilibrium inventory levels for the benchmark and asymmetric cases, with and without demand correlation (values normalized by $\alpha \mathbb{E}\left\{D_{2}+D_{1}\right\}$ ).

\subsection{Profit Comparison}

After analyzing the differences in inventory levels in equilibrium, we study the corresponding retailers' profits for the demand signal and mid-season replenishment cases. Figure 8 illustrates the increase of the retailers' equilibrium expected profits as a function of the demand correlation across time. The figure compares these profits to the benchmark case, SR vs. SR. In each graph, three curves appear: at the top, the profits of a QR retailer competing against a SR retailer; in the middle, the profits of a $\mathrm{QR}$ retailer competing against a QR retailer; and at the bottom, the profits of a SR retailer competing against a QR retailer. The two graphs on top correspond to the demand signal case, and the two in the bottom correspond to the mid-season replenishment. Likewise, the graphs at the left have $\sigma=0.3$ and the two at the right have $\sigma=0.6$. In terms of cost we use $c^{Q R}=c^{S R}=0.5 .^{15}$

Several observations can be made from looking at any of the graphs in Figure 8. First, since the bottom curve is positive, it implies that a SR retailer prefers to have a QR competitor (intuitively, the former gains from the spill-over demand that occurs in the initial period). Second, comparing the bottom and the middle curves shows that a SR retailer competing against a QR opponent would rather be QR itself. This confirms that flexibility pays off. Third, comparing the middle and the top curves shows that a QR retailer would rather have a SR competitor. Thus, part of the competitive advantage of QR comes from the replenishment agility asymmetry. The previous remarks are summarized in the following

\footnotetext{
${ }^{15}$ Again, the demand signal case might have multiple equilibria, but they all achieve the same profits (c.f. $\S 4.2 .1)$.
} 

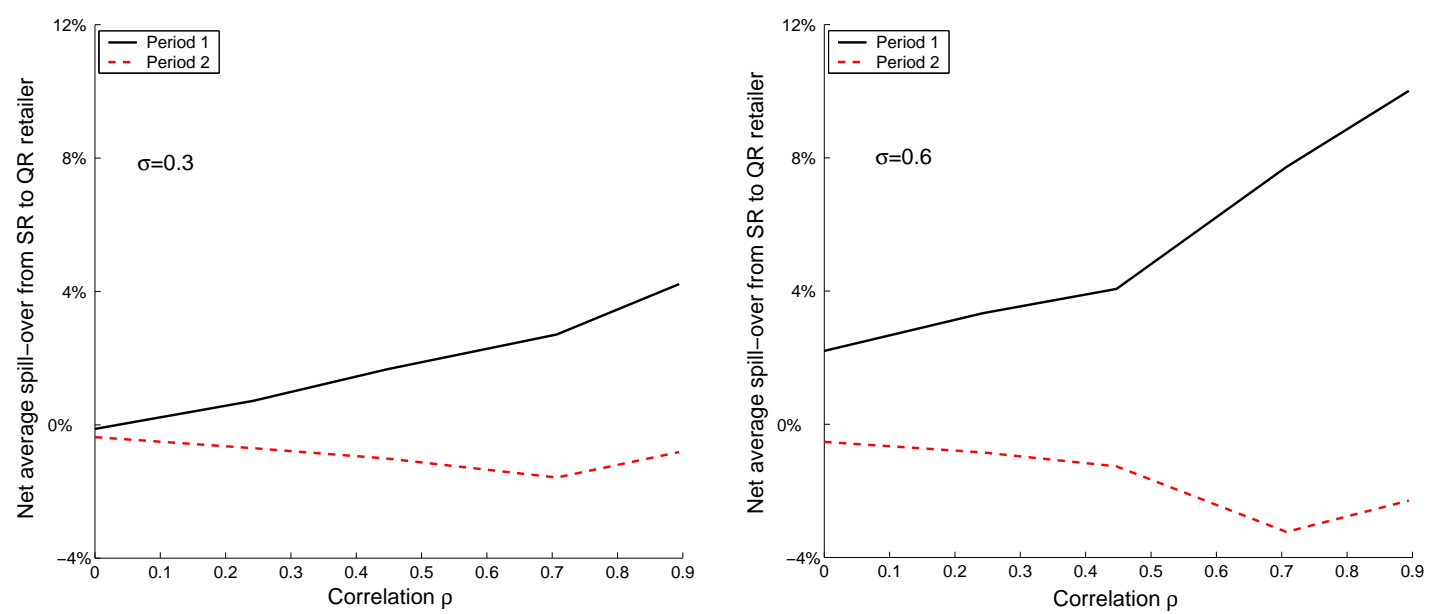

Figure 7: Net average spill-over from the SR retailer to the QR retailer (values normalized by $\left.\alpha \mathbb{E}\left\{D_{2}+D_{1}\right\}\right)$, for $\sigma=0.3$ and $\sigma=0.6$ ( $c^{Q R}=c^{S R}=0.5$ in both graphs).

preference ordering, where $\succcurlyeq$ represents a situation that is preferred by the first retailer:

$\mathrm{QR}$ vs. $\mathrm{SR} \succcurlyeq \mathrm{QR}$ vs. $\mathrm{QR} \succcurlyeq \mathrm{SR}$ vs. $\mathrm{QR} \succcurlyeq \mathrm{SR}$ vs. $\mathrm{SR}$.

Figure 8 also shows that the equilibrium profit increase due to the QR capability is larger when the correlation across periods and/or the demand uncertainty is larger. In the midseason replenishment case, it can generate up to $50 \%$ higher expected profits. In the demand signal case, the increase is lower, especially for low levels of demand correlation. This is what should be expected since there is significantly less demand spill-over (see the comment at the end of $\S 5.1$ ), and it indicates that in the demand signal case the benefit of QR is mostly explained by reduced under and overstock costs rather than capturing additional demand that spills over from the competitor.

The results of Figure 8 analyzed the increase in equilibrium profit when $c^{Q R}=c^{S R}$. However, as $c^{Q R}$ increases, the QR profit clearly decreases. We can thus compute the breakeven cost for which a retailer would be indifferent between being QR or SR. This result is depicted in Figure 9 as the percentage increase over $c^{S R}$ that leaves a retailer indifferent (while competing against a SR opponent). We plot the break-even costs as a function of the demand correlation, and for several values of $c^{S R}=0.3,0.5,0.7$. Again, the top and bottom graphs correspond to the demand signal and mid-season replenishment cases respectively, and the left and right graphs have $\sigma=0.3$ and $\sigma=0.6$ respectively.

Interestingly, while the SR retailer always gains when its competitor moves from SR to $\mathrm{QR}$, this is not always true for the QR retailer. It all depends on the cost increase associated 

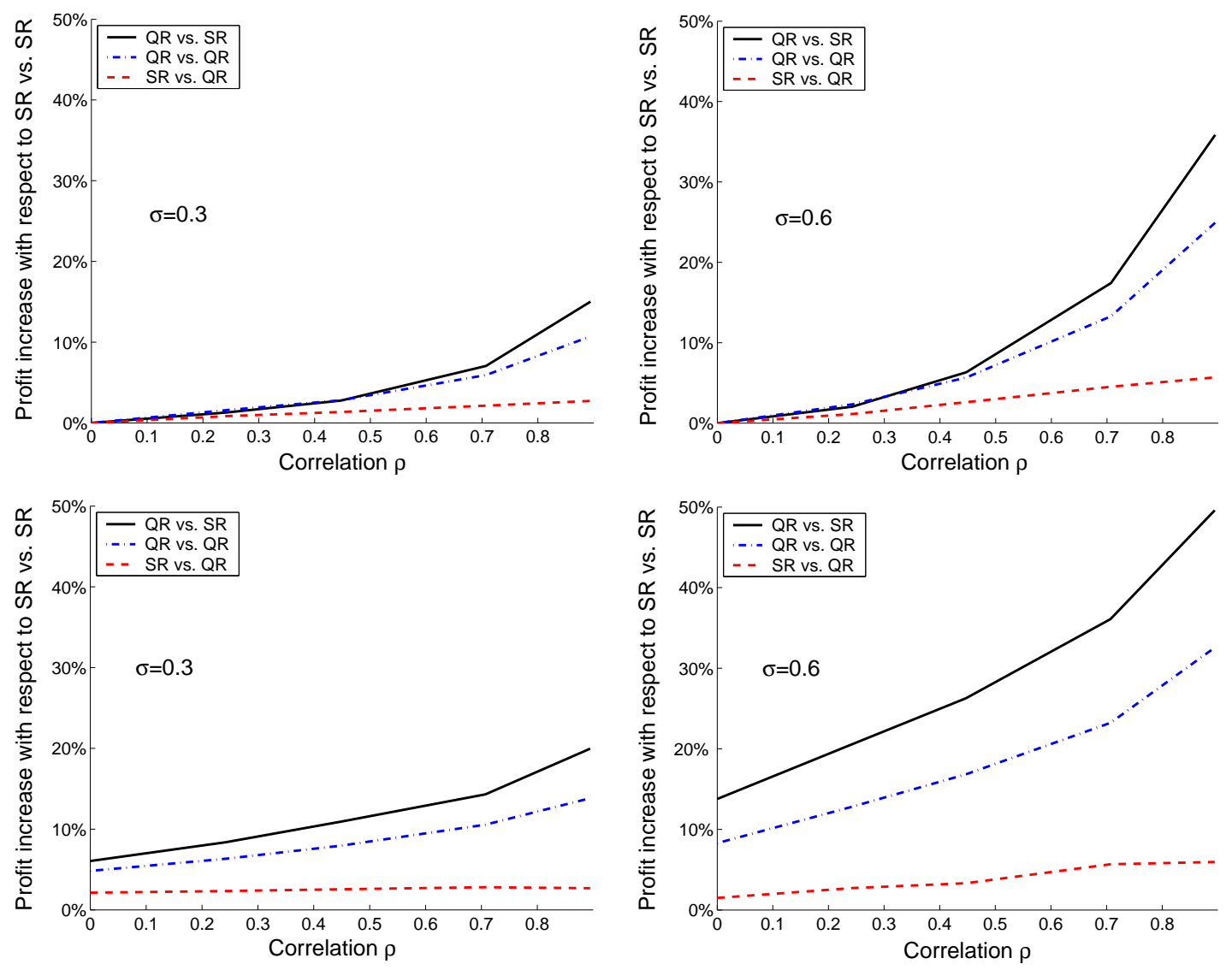

Figure 8: Increase in equilibrium profit compared to the benchmark scenario for the demand signal (top) and the mid-season replenishment (bottom) cases, with $c^{Q R}=c^{S R}=0.5$.

with implementing QR. For fast fashion retailers, the literature estimates this cost increase to be 15-20\% higher than traditional SR firms producing in Asia (see Ghemawat and Nueno 2003, p.11). As Figure 9 shows, this is insufficient to justify QR for small demand variability and/or small demand correlation across time. For example, in the mid-season replenishment model (bottom left), the break-even cost is below $15 \%$ for $\rho<0.5$ and $\sigma=0.3$. However, it can be much higher for $\rho \approx 0.9$ and $\sigma=0.6$. Thus, our model shows that for small demand variability and correlations, a retailer would rather prefer lower flexibility and lower cost, i.e., being SR. This applies for "basic" items, e.g., white T-shirts. On the other hand, for high demand variability and correlations, a retailer is better off having higher production cost but a faster response. This matches "fashion" goods, as those typically found in a Zara store. In other words, in a competitive setting, our results confirm the fundamental rule that the supply chain (in particular, its costs and flexibility) should match the type of product. Functional products, such as standard garments, should have an efficient (i.e, low cost, and usually less flexible) supply chain, whereas innovative product, such as trendy items, should 

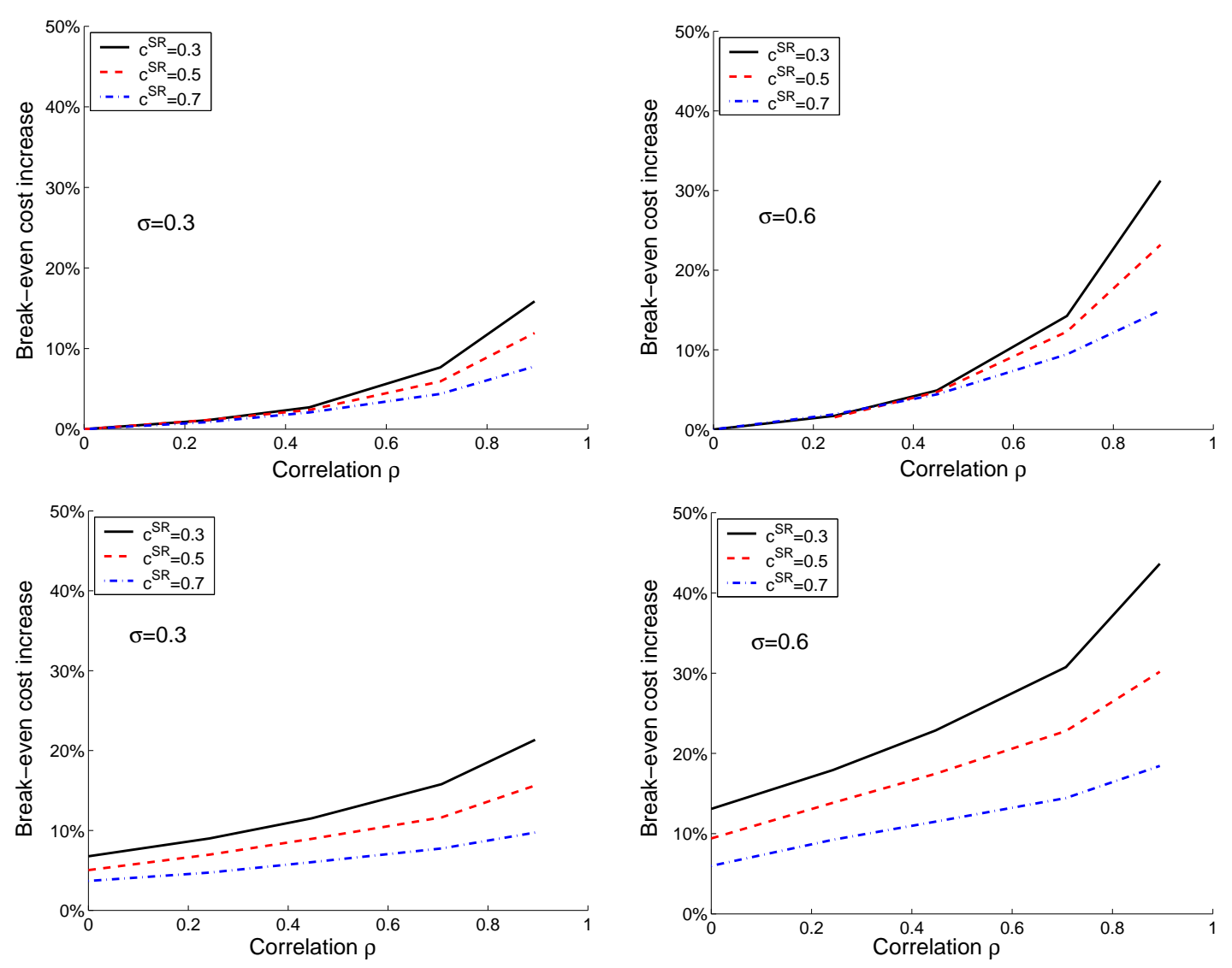

Figure 9: Break-even cost that makes a retailer (with a SR opponent) indifferent between being QR or SR, for the demand signal (top) and mid-season replenishment (bottom) cases.

have a supply chain that is responsive, which typically requires excess buffer capacity, and therefore implies higher operational costs (see Fisher 1997).

\section{Conclusions and Future Research}

In this paper, we formulated a multi-period finite horizon inventory competition model for two retailers selling substitutable items. The model can be used to analyze the impact of (asymmetric) production costs and ordering flexibility on the competitive outcome, and specifically on retailer inventory levels and profits. That is the case when one of the firms has a lower production (ordering) cost but can only produce at the beginning of the selling season, whereas the second firm has higher costs but can replenish stock during the planning horizon, taking advantage of any additional demand information that might become available. We visualize the problem as the competition between a traditional SR retailer that makes-tostock before the season starts, versus a QR firm that has a flexible supply chain and can order stock more than once. 
For the symmetric case, we extended the existence and unique equilibrium result by Lippman and McCardle (1997) to an arbitrary number of periods with demand learning and (possibly) nonzero initial inventory, where each firm follows a base-stock policy that ignores the competition. In other words, retailers adopt the same policy as if they were alone in the market. For asymmetric retailers and two periods $(T=2)$, we provided conditions that guarantees the existence of a unique pure-strategy subgame-perfect equilibrium for the demand signal and the mid-season replenishment cases. In addition, we performed an extensive numerical study to understand the impact of cost asymmetries, demand variability, and correlation across periods on the equilibrium inventory levels and the corresponding profits. One of the striking results of our model is that a SR retailer would rather compete against a $\mathrm{QR}$ retailer than a SR opponent. Also, a retailer competing against a SR retailer is better off being $\mathrm{QR}$, if the ordering $\operatorname{cost} c^{Q R}$ is below a break-even value that increases with demand uncertainty and the correlation across periods. Finally, we show that part of the competitive advantage for a $\mathrm{QR}$ retailer comes from the asymmetries on supply chain flexibility, since the increase in profits is higher in the QR vs. SR scenario than in the QR vs. QR case.

Several extensions of this work are possible. First, in terms of inventory competition models, ideal extensions include existence and unique equilibrium results for the asymmetric case with an arbitrary number of periods, and possibly more general demand allocation rules and a larger number of retailers. However, the analysis is presumably not straightforward. Second, in terms of understanding the fast fashion phenomenon, there is still plenty to be done. In fact, in this paper we have ignored other distinctive aspects such as the endogenous effect of higher fill rates on market share, similar to Gaur and Park (2007). Incorporating these elements into our model is a challenging strand of future research.

\section{Acknowledgments}

Both authors would like to thank Gerard Cachon, an associate editor, and three anonymous referees for their helpful and constructive comments on a previous version of this paper.

\section{References}

Anupindi, R. and Y. Bassok. 1999. "Centralization of Stocks: Retailers vs. Manufacturers." Management Science, 45(2), pp. 178-191. 
Avsar, Z.M. and M. Baykal-Gürsoy. 2002. "Inventory Control Under Substitutable Demand: a Stochastic Game Application." Naval Research Logistics, 49(4), pp. 359-375.

Bernstein, F. and A. Federgruen. 2004. "Dynamic Inventory and Pricing Models for Competing Retailers." Naval Research Logistics, 51(2), pp. 258-274.

Cachon, G. 2003. Supply Chain Coordination with Contracts, in Handbooks in Operations Research and Management Science: Supply Chain Management, edited by Steve Graves and Ton de Kok. North-Holland.

Cachon, G. and R. Swinney. 2007. Purchasing, Pricing, and Quick Response in the Presence of Strategic Customers. Working paper. The Wharton School, University of Pennsylvania.

Cachon, G. and C. Terwiesch. 2005. Matching Supply with Demand: An Introduction to Operations Management. McGraw-Hill/Irwin.

Caro, F. and J. Gallien. 2007. "Dynamic Assortment with Demand Learning for Seasonal Consumer Goods." Management Science, 53(2), pp. 276-292

Davidson, J. 2005. "Chic Thrills." Scotland on Sunday, February 13 issue.

Economist. 2005. "The future of fast fashion." The Economist, June 16th 2005 issue.

Ferdows, K., M. Lewis and J. A.D. Machuca. 2004. "Rapid-Fire Fullfilment." Harvard Business Review, November issue, Reprint R0411G.

Fisher, M. L. and A. Raman. 1996. Reducing the Cost of Demand Uncertainty Through Accurate Response to Early Sales. Operations Research, 44(1) 87-99.

Fisher, M. L. 1997. What Is the Right Supply Chain for Your Product? Harvard Business Review, March-April issue, Reprint 97205.

Foroohar, R. 2006. "A New Fashion Frontier." Newsweek International, March 20 issue.

Fudenberg, D. and J. Tirole 1991. Game Theory. MIT Press, Cambridge, Massachusetts.

Ghemawat, P. and J. L. Nueno. 2003. "ZARA: Fast Fashion." Harvard Business School Multimedia Case 9-703-416.

Gaur, V. and Y. Park. 2007. "Asymmetric Consumer Learning and Inventory Competition." Management Science, 53(2), pp. 227-240.

Hall, J. and E. Porteus. 2000. "Customer Service Competition in Capacitated Systems Source." Manufacturing and Service Operations Management, 2(2), pp. 144-165.

Hammond, J.H. and M.G. Kelly. 1990. "Quick Response in the Apparel Industry." Harvard 
Business School Note 9-690-038.

Iyer, A.V. and M.E. Bergen. 1997. "Quick Response in Manufacturer-Retailer Channels", Management Science, 43(4), pp. 559-570.

Kirman, A.P. and M.J. Sobel. 1974. "Dynamic Oligopoly with Inventories." Econometrica, 42(2), pp. 279-287.

Li, L. 1992. "The Role of Inventory in Delivery-Time Competition." Management Science, 38(2), pp. 182-197.

Lippman, S. A. and K. F. McCardle. 1997. "The Competitive Newsboy." Operations Research, 45(1), pp. 54-65.

Liu, L., W. Shang, and S. Wu. 2007. "Dynamic Competitive Newsvendors with ServiceSensitive Demands." Manufacturing and Service Operations Management, 9(1), pp. 8493.

Mahajan, S. and G. van Ryzin. 2001. "Inventory Competition Under Dynamic Consumer Choice." Operations Research, 49(3), pp. 646-657.

McAfee, A., V. Dessain and A. Sjöman. 2004. "ZARA: IT for Fast Fashion." Harvard Business School Case 9-604-081.

Nagarajan, M. and S. Rajagopalan. 2005. "Inventory Models for Substitutable Products: Monopoly and Duopoly Analysis." Working paper, Marshall School of Business, U.S.C.

Netessine, S. and N. Rudi. 2003. "Centralized and Competitive Inventory Models with Demand Substitution." Operations Research, 51(2), pp. 329-335.

Netessine, S. and F. Zhang. 2005. "Positive vs. Negative Externalities in Inventory Management: Implications for Supply Chain Design." Manufacturing and Service Operations Management, 7(1), pp. 58-73.

Netessine, S., N. Rudi and Y. Wang. 2006. "Inventory Competition and Incentives to Back-Order." IIE Transactions 38(11), pp. 883-902.

Olsen, T.L. and R.P. Parker. 2006. "Consumer Behavior in Inventory Management." Working paper, Washington University in St Louis.

Parlar, M. 1988. "Game Theoretic Analysis of the Substitutable Product Inventory Problem with Random Demands." Naval Research Logistics, 35(3), pp. 397-409.

Sobel, M. 1981. "Myopic Solutions of Markov Decision Processes and Stochastic Games", Operations Research, 29(5), pp. 995-1009. 


\title{
Technical Appendix to "The Impact of Quick Response in Inventory-Based Competition"
}

\author{
Felipe Caro • Victor Martínez-de-Albéniz \\ UCLA Anderson School of Management 110, Westwood Plaza, Suite B420 Los Angeles, \\ CA 90095, USA \\ IESE Business School University of Navarra Av. Pearson 2108034 Barcelona, Spain \\ fcaro@anderson.ucla.edu•valbeniz@iese.edu
}

October 28, 2007

\section{General Remarks:}

- The numbering of the equations in the appendix continues the same sequence from the paper.

- Throughout the appendix, any interchange of integration and differentiation in definite integrals is justified by Leibniz' rule, which holds whenever the integrand is continuously differentiable (almost everywhere).

\section{Proof of Proposition 1}

Proof. We omit $I_{1}$ since it remains constant throughout the proof. We can omit the subindex $t=1$ as well, since all the functions and variables in the proof refer to the terminal period. For each $y^{i}, y^{j}$, we use the following notation. Let $\kappa^{i}\left(y^{i}\right)=\left(q^{i}\right)^{-1}\left(y^{i}\right)$ and $\kappa^{j}\left(y^{j}\right)=\left(q^{j}\right)^{-1}\left(y^{j}\right)$. Each of these quantities represents the demand at which each retailer stocks out $(\kappa$ stands for knocked-out). Note that if $\kappa^{i}\left(y^{i}\right) \geq \kappa^{j}\left(y^{j}\right)$, then there can only be a spill-over of customers from $j$ to $i$. In addition, let

$$
\begin{aligned}
& \beta^{i}\left(y^{i}, y^{j}\right)=\mathbb{P}\left\{y^{i} \leq R^{i}\left(y^{j}\right)\right\}=1_{\kappa^{i}\left(y^{i}\right) \geq \kappa^{j}\left(y^{j}\right)} \int_{y^{i}+y^{j}}^{\infty} f(u) d u+1_{\kappa^{i}\left(y^{i}\right)<\kappa^{j}\left(y^{j}\right)} \int_{\kappa^{i}\left(y^{i}\right)}^{\infty} f(u) d u \text { and } \\
& \beta^{j}\left(y^{i}, y^{j}\right)=\mathbb{P}\left\{\kappa^{j}\left(y^{j}\right) \leq D \leq y^{i}+y^{j}\right\}=1_{\kappa^{i}\left(y^{i}\right) \geq \kappa^{j}\left(y^{j}\right)} \int_{\kappa^{j}\left(y^{j}\right)}^{y^{i}+y^{j}} f(u) d u,
\end{aligned}
$$

where $1_{A}$ is the indicator function of event $A$. 
Following Figure 1, let $E^{i}$ and $E^{j}$ be the equilibrium stocking quantities in the unconstrained competitive game, i.e, when both retailers start without any inventory. Similarly, let $s^{i}\left(y^{j}\right)$ and $s^{j}\left(y^{i}\right)$ be the unconstrained base-stock functions. From the definition of a Nash equilibrium, $E^{i}=s^{i}\left(E^{j}\right)$ and $E^{j}=s^{j}\left(E^{i}\right)$, and we have that $e^{i}(0,0)=E^{i}$ and $e^{j}(0,0)=E^{j}$. We now compute the partial derivatives of $\pi^{i}$ in each one of the four regions (I)-(IV) depicted in Figure 1.

(I) When $x^{i} \leq E^{i}, x^{j} \leq E^{j}$, then $e^{i}=E^{i}$ and $e^{j}=E^{j}$. Hence, from Equation (4), $\pi^{i}$ is linear in $x^{i}$, with $\frac{1}{p^{i}} \frac{\partial \pi^{i}}{\partial x^{i}}=\frac{c^{i}}{p^{i}}$ and independent of $x^{j}$.

(II) When $x^{j} \geq E^{j}, x^{i} \leq s^{i}\left(x^{j}\right)$, then $e^{j}=x^{j}$ and $e^{i}=s^{i}\left(x^{j}\right)$. Hence, $\pi^{i}=c^{i} x^{i}-c^{i} s^{i}\left(x^{j}\right)+$ $p^{i} \mathbb{E} \min \left\{s^{i}\left(x^{j}\right), R^{i}\left(x^{j}\right)\right\}$. Then, we have that

$$
\frac{1}{p^{i}} \frac{\partial \pi^{i}}{\partial x^{i}}=\frac{c^{i}}{p^{i}} \text { and } \frac{1}{p^{i}} \frac{\partial \pi^{i}}{\partial x^{j}}=-\mathbb{P}\left\{\kappa^{j}\left(x^{j}\right) \leq D \leq s^{i}\left(x^{j}\right)+x^{j}\right\}=-\beta^{j}\left(e^{i}, e^{j}\right)
$$

where the latter follows from the envelope theorem.

(III) When $x^{i} \geq E^{i}, x^{j} \leq s^{j}\left(x^{i}\right)$, then $e^{i}=x^{i}$ and $e^{j}=s^{j}\left(x^{i}\right)$. Hence, $\pi^{i}=p^{i} \mathbb{E} \min \left\{x^{i}, R^{i}\left(s^{j}\left(x^{i}\right)\right)\right\}$. It is independent of $x^{j}$ and

$$
\begin{aligned}
\frac{1}{p^{i}} \frac{\partial \pi^{i}}{\partial x^{i}} & =\mathbb{P}\left\{x^{i} \leq R^{i}\left(s^{j}\left(x^{i}\right)\right)\right\}-\frac{d s^{j}}{d y^{i}} \mathbb{P}\left\{\kappa^{j}\left(s^{j}\left(x^{i}\right)\right) \leq D \leq x^{i}+s^{j}\left(x^{i}\right)\right\} \\
& =\beta^{i}\left(x^{i}, s^{j}\left(x^{i}\right)\right)-\beta^{j}\left(x^{i}, s^{j}\left(x^{i}\right)\right) \frac{d s^{j}}{d y^{i}}
\end{aligned}
$$

Notice that, from the implicit function theorem at $y^{j}=s^{j}\left(y^{i}\right)$, we have that

$$
\frac{d s^{j}}{d y^{i}}=\frac{\frac{\partial^{2} r^{j}}{\partial y^{j} \partial y^{i}}}{-\frac{\partial^{2} r^{j}}{\left(\partial y^{j}\right)^{2}}}=-1_{\kappa^{i}\left(y^{i}\right)<\kappa^{j}\left(s^{j}\left(y^{i}\right)\right)}
$$

That is, when the derivative of the base-stock function $s^{j}$ is not zero then $\beta^{j}\left(y^{i}, s^{j}\left(y^{i}\right)\right)$ is zero. In other words, $\beta^{j}\left(y^{i}, s^{j}\left(y^{i}\right)\right) \frac{d s^{j}}{d y^{i}}$ is always equal to zero. Hence,

$$
\frac{1}{p^{i}} \frac{\partial \pi^{i}}{\partial x^{i}}=\beta^{i}\left(e^{i}, e^{j}\right)
$$

(IV) When both retailers start with inventories above their equilibrium quantities, then $e^{i}=x^{i}, e^{j}=x^{j}$, and $\pi^{i}=p^{i} \mathbb{E} \min \left\{x^{i}, R^{i}\left(x^{j}\right)\right\}$. Then,

$$
\frac{1}{p^{i}} \frac{\partial \pi^{i}}{\partial x^{i}}=\mathbb{P}\left\{x^{i} \leq R^{i}\left(x^{j}\right)\right\}=\beta^{i}\left(e^{i}, e^{j}\right)
$$




\begin{tabular}{|c|c|c|}
\hline Region & $\frac{1}{p^{i}} \frac{\partial \pi^{i}}{\partial x^{i}}$ & $\frac{1}{p^{i}} \frac{\partial \pi^{i}}{\partial x^{j}}$ \\
\hline (I) & $\frac{c^{i}}{p^{i}}$ & 0 \\
\hline (II) & $\frac{c^{i}}{p^{i}}$ & $-\beta^{j}\left(s^{i}\left(x^{j}\right), x^{j}\right)$ \\
\hline (III) & $\beta^{i}\left(x^{i}, s^{j}\left(x^{i}\right)\right)$ & 0 \\
\hline (IV) & $\beta^{i}\left(x^{i}, x^{j}\right)$ & $-\beta^{j}\left(x^{i}, x^{j}\right)$ \\
\hline
\end{tabular}

Table 3: Properties of $\pi_{1}^{i}$.

and

$$
\frac{1}{p^{i}} \frac{\partial \pi^{i}}{\partial x^{j}}=-\mathbb{P}\left\{\kappa^{j}\left(x^{j}\right) \leq D \leq x^{i}+x^{j}\right\}=-\beta^{j}\left(e^{i}, e^{j}\right)
$$

We summarize these findings in Table 3. Note that $\pi^{i}$ is continuous in $x^{i}, x^{j}$, and is differentiable almost everywhere. In addition, note that $\frac{\partial \pi^{i}}{\partial x^{j}}$ is continuous in $x^{i}$ but discontinuous in $x^{j}$ in the border of regions (I) and (II) .

Interestingly, $\frac{\partial \pi^{i}}{\partial x^{i}}$ is continuous in both arguments. In particular, taking $x^{i}$ fixed, and increasing $x^{j}$, we move from either region (I) to (II), or from region (I) to (II) to (IV), or from region (III) to (IV). In each one of borders, $\frac{\partial \pi^{i}}{\partial x^{i}}$ is continuous, as $\beta^{i}=\frac{c^{i}}{p^{i}}$ in the border (II)-(IV) and $s^{j}\left(x^{i}\right)=x^{j}$ in the border (III)-(IV). Similarly, taking $x^{j}$ fixed, and increasing $x^{i}$, we move from either region (I) to (III), or from region (I) to (III) to (IV), or from region (II) to (IV). Again, there is no jump in $\frac{\partial \pi^{i}}{\partial x^{i}}$ as we move from region to region. Since it is non-increasing as a function of $x^{i}$ inside the regions, $\pi^{i}$ is concave in $x^{i}$.

\section{Proof of Proposition 2}

Proof. As before, for simplicity we omit the subscript " $1 \mid D_{2}$ " and use subscript "1" instead. The proof is quite long, but the main idea is rather simple. We want to show that the following inequality holds

$$
\frac{\partial^{2} r_{2}^{i}}{\left(\partial y_{2}^{i}\right)^{2}}<\phi_{2}\left(\left(q_{2}^{i}\right)^{-1}\left(y_{2}^{i}\right)\right)\left(\frac{\partial r_{2}^{i}}{\partial y_{2}^{i}}\right), \text { where } \phi_{2}(y)=\max \left\{0, \frac{f_{2}^{\prime}}{f_{2}}(y)\right\}
$$

This is the same inequality as (10), and for expositional purposes, first assume that it actually holds (we will prove it shortly). Then, consider a critical point of $r_{2}^{i}\left(\cdot, y_{2}^{j}\right)$, i.e., $\frac{\partial r_{2}^{i}}{\partial y_{2}^{i}}\left(y_{2}^{i}, y_{2}^{j}\right)=0$. From inequality (16), the critical point is necessarily a strict maximum, i.e., $\frac{\partial^{2} r_{2}^{i}}{\left(\partial y_{2}^{i}\right)^{2}}\left(y_{2}^{i}, y_{2}^{j}\right)<0$. This shows that $r_{2}^{i}\left(\cdot, y_{2}^{j}\right)$ is first increasing and then decreasing, i.e., 
quasi-concave. In addition, for $y_{2}^{i}$ very large, $r_{2}^{i}$ is eventually decreasing. Hence, there exists a maximizer of $r_{2}^{i}\left(\cdot, y_{2}^{j}\right)$. Furthermore, this maximizer must be unique. In fact, consider two distinct maxima. By (16), both would have to be strict. This would imply that in between them, there would have to be a minimum, which again would be a contradiction with (16).

As a result, denote $s_{2}^{i}\left(y_{2}^{j}\right)$ the unique maximizer of $r_{2}^{i}\left(y_{2}^{i}, y_{2}^{j}\right)$. From quasi-concavity, the optimal (unconstrained) policy is base-stock, and from Equation (6), the constrained best response is $b_{2}^{i}\left(x_{2}^{i}, y_{2}^{j}\right)=\max \left\{x_{2}^{i}, s_{2}^{i}\left(y_{2}^{j}\right)\right\}$. Therefore, to prove Proposition 2 it suffices to show that the inequality (16) is valid. That is what we do next.

We start the proof of (16) using the notation and results from Table 3. We structure the proof in three steps: in claims 1 and 2 , we provide bounds on $\frac{\partial^{2} \pi_{1}^{i}}{\left(\partial x_{1}^{i}\right)^{2}}, \frac{\partial \pi_{1}^{i}}{\partial x_{1}^{j}}$ and $\frac{\partial \pi_{1}^{i}}{\partial x_{1}^{i}}$; then, in claim 3, we finally prove the inequality (16).

Claim 1 Letting

$$
\phi_{1}\left(x_{1}^{i}\right)=\max \left\{0, \frac{f_{1}^{\prime}}{f_{1}}\left(x_{1}^{i}\right)\right\}
$$

we have that $\frac{\partial^{2} \pi_{1}^{i}}{\left(\partial x_{1}^{i}\right)^{2}} \leq-\phi_{1}\left(\left(q_{1}^{i}\right)^{-1}\left(x_{1}^{i}\right)\right)\left(c_{1}^{i}-\frac{\partial \pi_{1}^{i}}{\partial x_{1}^{i}}\right)$.

We prove the claim for each one of the four regions in Figure 1. As in the previous proof, we use the notation of Equation (15) and let $\kappa_{1}^{i}\left(y_{1}^{i}\right)=\left(q_{1}^{i}\right)^{-1}\left(y_{1}^{i}\right)$ and $\kappa_{1}^{j}\left(y_{1}^{j}\right)=\left(q_{1}^{j}\right)^{-1}\left(y_{1}^{j}\right)$.

(I) $\frac{\partial^{2} \pi_{1}^{i}}{\left(\partial x_{1}^{i}\right)^{2}}=0 \cdot\left(c_{1}^{i}-\frac{\partial \pi_{1}^{i}}{\partial x_{1}^{i}}\right)$.
(II) $\frac{\partial^{2} \pi_{1}^{i}}{\left(\partial x_{1}^{i}\right)^{2}}=0 \cdot\left(c_{1}^{i}-\frac{\partial \pi_{1}^{i}}{\partial x_{1}^{i}}\right)$.

(IV) We leave case (III) for the end. Here, using Equation (15), we have

$$
\beta^{i}\left(y_{1}^{i}, y_{1}^{j}\right)=1_{\kappa_{1}^{i} \geq \kappa_{1}^{j}} \int_{y_{1}^{i}+y_{1}^{j}}^{\infty} f_{1}(u) d u+1_{\kappa_{1}^{i}<\kappa_{1}^{j}} \int_{\kappa_{1}^{i}}^{\infty} f_{1}(u) d u
$$

and thus, noting that $\frac{d \kappa_{1}^{i}}{d y_{1}^{i}}=\frac{1}{\left(q_{1}^{i}\right)^{\prime}\left(\kappa_{1}^{i}\right)}$,

$$
\frac{\partial \beta^{i}}{\partial y_{1}^{i}}=-1_{\kappa_{1}^{i} \geq \kappa_{1}^{j}} f_{1}\left(y_{1}^{i}+y_{1}^{j}\right)-1_{\kappa_{1}^{i}<\kappa_{1}^{j}} \frac{f_{1}\left(\kappa_{1}^{i}\right)}{\left(q_{1}^{i}\right)^{\prime}\left(\kappa_{1}^{i}\right)}
$$

Since $f_{1}$ is $\log$-concave, i.e., $\log \left(f_{1}\right)$ is concave, $F_{1}$ is also log-concave, and for all $v$ in the support of $f_{1}$,

$$
\frac{f_{1}(v)}{F_{1}(v)} \geq \max \left\{0, \frac{f_{1}^{\prime}(v)}{f_{1}(v)}\right\}
$$


see Bagnoli and Bergstrom (2005) or Martínez-de-Albéniz (2004).

Thus, we have, using that $\left(q_{1}^{i}\right)^{\prime} \leq 1$ (because $\left(q_{1}^{j}\right)^{\prime}=1-\left(q_{1}^{i}\right)^{\prime} \geq 0$ ),

$$
\begin{aligned}
& -\frac{\partial \beta^{i}}{\partial y^{i}} \geq 1_{\kappa_{1}^{i} \geq \kappa_{1}^{j}} f_{1}\left(y_{1}^{i}+y_{1}^{j}\right)+1_{\kappa_{1}^{i}<\kappa_{1}^{j}} f_{1}\left(\kappa_{1}^{i}\right) \\
& \geq 1_{\kappa_{1}^{i} \geq \kappa_{1}^{j}} \max \left\{0, \frac{f_{1}^{\prime}}{f_{1}}\left(y_{1}^{i}+y_{1}^{j}\right)\right\} \int_{0}^{y_{1}^{i}+y_{1}^{j}} f_{1}(u) d u \\
& +1_{\kappa_{1}^{i}<\kappa_{1}^{j}} \max \left\{0, \frac{f_{1}^{\prime}}{f_{1}}\left(\kappa_{1}^{i}\right)\right\} \int_{0}^{\kappa_{1}^{i}} f_{1}(u) d u \\
& =\max \left\{0, \frac{f_{1}^{\prime}}{f_{1}}\left(y_{1}^{i}+y_{1}^{j}\right), \frac{f_{1}^{\prime}}{f_{1}}\left(\kappa_{1}^{i}\right)\right\} \\
& \times\left(\begin{array}{c}
1_{\kappa_{1}^{i} \geq \kappa_{1}^{j}}\left(1-\int_{y_{1}^{i}+y_{1}^{j}}^{\infty} f_{1}(u) d u\right) \\
+1_{\kappa_{1}^{i}<\kappa_{1}^{j}}\left(1-\int_{\kappa_{1}^{i}}^{\infty} f_{1}(u) d u\right)
\end{array}\right) \\
& \geq \max \left\{0, \frac{f_{1}^{\prime}}{f_{1}}\left(\left(q_{1}^{i}\right)^{-1}\left(y_{1}^{i}\right)\right)\right\}\left(\frac{c_{1}^{i}}{p_{1}^{i}}-\beta^{i}\right) \text {. }
\end{aligned}
$$

This can be rewritten as

$$
\frac{\partial^{2} \pi_{1}^{i}}{\left(\partial x_{1}^{i}\right)^{2}} \leq-\max \left\{0, \frac{f_{1}^{\prime}}{f_{1}}\left(\left(q_{1}^{i}\right)^{-1}\left(x_{1}^{i}\right)\right)\right\}\left(c_{1}^{i}-\frac{\partial \pi_{1}^{i}}{\partial x_{1}^{i}}\right)
$$

(III) We have

$$
\frac{1}{p_{1}^{i}} \frac{\partial^{2} \pi_{1}^{i}}{\left(\partial x_{1}^{i}\right)^{2}}=\frac{\partial \beta^{i}}{\partial y^{i}}+\frac{\partial \beta^{i}}{\partial y^{j}} \frac{d s_{1}^{j}}{d y_{1}^{i}}=\frac{\partial \beta^{i}}{\partial y^{i}}
$$

This is true because, when $\frac{d s_{1}^{j}}{d y_{1}^{i}} \neq 0$, then $\kappa_{1}^{i}\left(y_{1}^{i}\right) \leq \kappa_{1}^{j}\left(s_{1}^{j}\right)$. This implies that $\beta^{i}\left(y_{1}^{i}, s_{1}^{j}\right)=$ $\int_{\kappa_{1}^{i}}^{\infty} f_{1}(u) d u$, and hence $\frac{\partial \beta^{i}}{\partial y^{j}}=0$ at $\left(y_{1}^{i}, s_{1}^{j}\right)$. Similarly to case (IV),

$$
\frac{\partial^{2} \pi_{1}^{i}}{\left(\partial x_{1}^{i}\right)^{2}} \leq-\max \left\{0, \frac{f_{1}^{\prime}}{f_{1}}\left(\left(q_{1}^{i}\right)^{-1}\left(x_{1}^{i}\right)\right)\right\}\left(c_{1}^{i}-\frac{\partial \pi_{1}^{i}}{\partial x_{1}^{i}}\right) .
$$

This completes the proof of the bound. We need a second bound on the first derivatives.

Claim $2 \frac{\partial \pi_{1}^{i}}{\partial x_{1}^{i}} \leq c_{1}^{i}$; for all $x^{j}$ such that $\bar{F}_{1}\left(\left(q_{1}^{j}\right)^{-1}\left(x_{1}^{j}\right)\right)<1, \frac{\partial \pi_{1}^{i}}{\partial x_{1}^{j}}\left(0, x_{1}^{j}\right)>-\left(p_{1}^{i}-c_{1}^{i}\right)$. 
The first part of the claim, $\frac{\partial \pi_{1}^{i}}{\partial x_{1}^{i}} \leq c_{1}^{i}$, is a direct consequence of concavity with respect to $x_{1}^{i}$ and $\frac{\partial \pi_{1}^{i}}{\partial x_{1}^{i}}\left(0, x_{1}^{j}\right)=c_{1}^{i}$.

For the second part, we notice that $\left(0, x_{1}^{j}\right)$ belongs to regions (I) or (II). When $\frac{\partial \pi_{1}^{i}}{\partial x_{1}^{j}}\left(0, x_{1}^{j}\right) \neq$ 0 , we must be in region (II). There, $\frac{\partial \pi_{1}^{i}}{\partial x_{1}^{j}}\left(0, x_{1}^{j}\right)=-\beta^{j}\left(s_{1}^{i}\left(x_{1}^{j}\right), x_{1}^{j}\right)$. Recall that we have, at $y_{1}^{i}=s_{1}^{i}\left(x_{1}^{j}\right)$ (unconstrained best-response),

$$
\frac{d s_{1}^{i}}{d y_{1}^{j}}=\frac{\frac{\partial^{2} r_{1}^{i}}{\partial y_{1}^{i} \partial y_{1}^{j}}}{-\frac{\partial^{2} r_{1}^{i}}{\left(\partial y_{1}^{i}\right)^{2}}}=-1_{\kappa_{1}^{i}\left(s_{1}^{i}\right) \geq \kappa_{1}^{j}\left(x_{1}^{j}\right)}
$$

That is, $\beta^{j}\left(s_{1}^{i}\left(x_{1}^{j}\right), x_{1}^{j}\right)$ is not zero if and only if the best-response $s_{1}^{i}\left(x_{1}^{j}\right)=\bar{F}_{1}^{-1}\left(\frac{c_{1}^{i}}{p_{1}^{i}}\right)-x_{1}^{j}$, from Theorem 1. As a result, when $\frac{\partial \pi_{1}^{i}}{\partial x_{1}^{j}}\left(0, x_{1}^{j}\right) \neq 0$,

$$
\begin{aligned}
\frac{1}{p_{i}} \frac{\partial \pi_{1}^{i}}{\partial x_{1}^{j}}\left(0, x_{1}^{j}\right) & =-\beta^{j}\left(\bar{F}_{1}^{-1}\left(\frac{c_{1}^{i}}{p_{1}^{i}}\right)-x_{1}^{j}, x_{1}^{j}\right) \\
& =-1_{\kappa_{1}^{j}\left(x_{1}^{j}\right) \leq \bar{F}_{1}^{-1}\left(\frac{c_{1}^{i}}{p_{1}^{i}}\right)}\left(\int_{\kappa_{1}^{j}}^{\bar{F}_{1}^{-1}\left(\frac{c_{1}^{i}}{p_{1}^{i}}\right)} f_{1}(u) d u\right) \\
& =-1_{\bar{F}_{1}\left(\kappa_{1}^{j}\right) \geq \frac{c_{1}^{i}}{p_{1}^{i}}}\left(\bar{F}_{1}\left(\kappa_{1}^{j}\right)-\frac{c_{1}^{i}}{p_{1}^{i}}\right)
\end{aligned}
$$

Finally, this expression is valid (otherwise it is zero) while we are in region (II): $x_{1}^{j}$ must be greater than $s_{1}^{j}\left(s_{1}^{i}\left(x_{1}^{j}\right)\right)$. We know that $s_{1}^{i}$ has slope -1 here, which implies that $s_{1}^{j}$ must be constant, since at the intersection at most one of the best-response curves has slope -1 . In other words, $x_{1}^{j} \geq q_{1}^{j}\left(\bar{F}_{1}^{-1}\left(\frac{c_{1}^{j}}{p_{1}^{j}}\right)\right)$. Hence, we can express

$$
\frac{1}{p_{i}} \frac{\partial \pi_{1}^{i}}{\partial x_{1}^{j}}\left(0, x_{1}^{j}\right)=-1_{\frac{c_{1}^{i}}{p_{1}^{i}} \leq \bar{F}_{1}\left(\left(q_{1}^{j}\right)^{-1}\left(x_{1}^{j}\right)\right) \leq \frac{c_{1}^{j}}{p_{1}^{j}}}\left(\bar{F}_{1}\left(\left(q_{1}^{j}\right)^{-1}\left(x_{1}^{j}\right)\right)-\frac{c_{1}^{i}}{p_{1}^{i}}\right)
$$

which completes the second part of the claim.

With these two claims we are ready to prove the key inequality (16).

Claim $3 \frac{\partial^{2} r_{2}^{i}}{\left(\partial y_{2}^{i}\right)^{2}}<\phi_{2}\left(\left(q_{2}^{i}\right)^{-1}\left(y_{2}^{i}\right)\right)\left(\frac{\partial r_{2}^{i}}{\partial y_{2}^{i}}\right)$, where

$$
\phi_{2}(y)=\max \left\{0, \frac{f_{2}^{\prime}}{f_{2}}(y)\right\} \text {. }
$$


For this purpose, take $y_{2}^{j}$ as fixed. Similarly as before, let $\kappa_{2}^{i}\left(y_{2}^{i}\right)=\left(q_{2}^{i}\right)^{-1}\left(y_{2}^{i}\right)$ and $\kappa_{2}^{j}\left(y_{2}^{j}\right)=\left(q_{2}^{j}\right)^{-1}\left(y_{2}^{j}\right)$. Equation (5) can be written as

$$
r_{2}^{i}\left(y_{2}^{i}, y_{2}^{j}\right)=\mathbb{E}\left\{\begin{array}{c}
-c_{2}^{i} y_{2}^{i}+p_{2}^{i} \min \left\{y_{2}^{i}, R_{2}^{i}\left(y_{2}^{j}\right)\right\} \\
+\pi_{1 \mid D_{2}}^{i}\left(\left(y_{2}^{i}-R_{2}^{i}\left(y_{2}^{j}\right)\right)^{+},\left(y_{2}^{j}-R_{2}^{j}\left(y_{2}^{i}\right)\right)^{+}\right)
\end{array}\right\}
$$

There are two possible situations given $y_{2}^{i}, y_{2}^{j}$. Either $j$ stocks out before $i$, and hence there may be a spill over from $j$ to $i$, in which case $\kappa_{2}^{i} \geq \kappa_{2}^{j}$; or vice-versa.

The first situation occurs when $\kappa_{2}^{i} \geq \kappa_{2}^{j}$. Here, noting that the integrand is continuously differentiable almost everywhere (and hence differentiation and integration can be interchanged),

$$
\begin{aligned}
\frac{\partial r_{2}^{i}}{\partial y_{2}^{i}}= & -c_{2}^{i}+p_{2}^{i} \mathbb{P}\left\{y_{2}^{i}+y_{2}^{j} \leq D_{2}\right\}+\mathbb{E}\left\{\frac{\partial \pi_{1 \mid D_{2}}^{i}}{\partial x_{1}^{i}}\left(y_{2}^{i}-q_{2}^{i}\left(D_{2}\right), y_{2}^{j}-q_{2}^{j}\left(D_{2}\right)\right) 1_{\kappa_{2}^{j} \geq D_{2}}\right\} \\
& +\mathbb{E}\left\{\frac{\partial \pi_{1 \mid D_{2}}^{i}}{\partial x_{1}^{i}}\left(y_{2}^{i}+y_{2}^{j}-D_{2}, 0\right) 1_{\kappa_{2}^{j} \leq D_{2} \leq y_{2}^{i}+y_{2}^{j}}\right\}
\end{aligned}
$$

Since $\pi_{1 \mid D_{2}}^{i}$ is concave in $x_{1}^{i}$, for all $D_{2}, r_{2}^{i}$ is concave in $y_{2}^{i}$. In fact, the second derivative can be expressed as

$$
\begin{aligned}
\frac{\partial^{2} r_{2}^{i}}{\left(\partial y_{2}^{i}\right)^{2}}= & -p_{2}^{i} f_{2}\left(y_{2}^{i}+y_{2}^{j}\right)+\mathbb{E}\left\{\frac{\partial^{2} \pi_{1 \mid D_{2}}^{i}}{\left(\partial x_{1}^{i}\right)^{2}}\left(y_{2}^{i}-q_{2}^{i}\left(D_{2}\right), y_{2}^{j}-q_{2}^{j}\left(D_{2}\right)\right) 1_{\kappa_{2}^{j} \geq D_{2}}\right\} \\
& +\mathbb{E}\left\{\frac{\partial^{2} \pi_{1 \mid D_{2}}^{i}}{\left(\partial x_{1}^{i}\right)^{2}}\left(y_{2}^{i}+y_{2}^{j}-D_{2}, 0\right) 1_{\kappa_{2}^{j} \leq D_{2} \leq y_{2}^{i}+y_{2}^{j}}\right\}+\frac{\partial \pi_{1 \mid D_{2}}^{i}}{\partial x_{1}^{i}}(0,0) f_{2}\left(y_{2}^{i}+y_{2}^{j}\right) \\
< & \mathbb{E}\left\{\phi_{1 \mid u}\left(\left(q_{1}^{i}\right)^{-1}\left(y_{2}^{i}-q_{2}^{i}\left(D_{2}\right)\right)\right)\left(\frac{\partial \pi_{1 \mid D_{2}}^{i}}{\partial x_{1}^{i}}\left(y_{2}^{i}-q_{2}^{i}\left(D_{2}\right), y_{2}^{j}-q_{2}^{j}\left(D_{2}\right)\right)-c_{1}^{i}\right) 1_{\kappa_{2}^{j} \geq D_{2}}\right\} \\
& +\mathbb{E}\left\{\phi_{1 \mid u}\left(\left(q_{1}^{i}\right)^{-1}\left(y_{2}^{i}+y_{2}^{j}-D_{2}\right)\left(\frac{\partial \pi_{1 \mid D_{2}}^{i}}{\partial x_{1}^{i}}\left(y_{2}^{i}+y_{2}^{j}-D_{2}, 0\right)-c_{1}^{i}\right) 1_{\kappa_{2}^{j} \leq D_{2} \leq y_{2}^{i}+y_{2}^{j}}\right\}\right.
\end{aligned}
$$

(using Claims 1 and 2, and the infinite support of $D_{2}$ )

$$
\begin{aligned}
\leq & \mathbb{E}\left\{\phi_{2}\left(\left(q_{2}^{i}\right)^{-1}\left(y_{2}^{i}\right)\right)\left(\frac{\partial \pi_{1 \mid D_{2}}^{i}}{\partial x_{1}^{i}}\left(y_{2}^{i}-q_{2}^{i}\left(D_{2}\right), y_{2}^{j}-q_{2}^{j}\left(D_{2}\right)\right)-c_{1}^{i}\right) 1_{\kappa_{2}^{j} \geq D_{2}}\right\} \\
& +\mathbb{E}\left\{\phi_{2}\left(\left(q_{2}^{i}\right)^{-1}\left(y_{2}^{i}\right)\right)\left(\frac{\partial \pi_{1 \mid D_{2}}^{i}}{\partial x_{1}^{i}}\left(y_{2}^{i}+y_{2}^{j}-D_{2}, 0\right)-c_{1}^{i}\right) 1_{\kappa_{2}^{j} \leq D_{2} \leq y_{2}^{i}+y_{2}^{j}}\right\}
\end{aligned}
$$

(using that $\phi_{1 \mid u}(x)=\max \left\{0, \frac{g^{\prime}}{g}(x-k u)\right\} \geq \phi_{2}(x-k u) \geq \phi_{2}(x)$,

from assumption (iii) and that $\phi_{2}$ is non-increasing, assumption (ii))

$$
\begin{aligned}
& \leq \phi_{2}\left(\left(q_{2}^{i}\right)^{-1}\left(y_{2}^{i}\right)\right)\left(\frac{\partial r_{2}^{i}}{\partial y_{2}^{i}}-\left(c_{1}^{i}-c_{2}^{i}\right)-\left(p_{2}^{i}-c_{1}^{i}\right) \mathbb{P}\left\{y_{2}^{i}+y_{2}^{j} \leq D_{2}\right\}\right) \\
& \leq \phi_{2}\left(\left(q_{2}^{i}\right)^{-1}\left(y_{2}^{i}\right)\right)\left(\frac{\partial r_{2}^{i}}{\partial y_{2}^{i}}\right) .
\end{aligned}
$$


Consider now the second situation, i.e., $\kappa_{2}^{i}<\kappa_{2}^{j}$. Here, since there is only spill-over from $i$ to $j$,

$$
\begin{aligned}
\frac{\partial r_{2}^{i}}{\partial y_{2}^{i}}= & -c_{2}^{i}+p_{2}^{i} \mathbb{P}\left\{\kappa_{2}^{i} \leq D_{2}\right\}+\mathbb{E}\left\{\frac{\partial \pi_{1 \mid D_{2}}^{i}}{\partial x_{1}^{i}}\left(y_{2}^{i}-q_{2}^{i}\left(D_{2}\right), y_{2}^{j}-q_{2}^{j}\left(D_{2}\right)\right) 1_{\kappa_{2}^{i} \geq D_{2}}\right\} \\
& +\mathbb{E}\left\{\frac{\partial \pi_{1 \mid D_{2}}^{i}}{\partial x_{1}^{j}}\left(0, y_{2}^{i}+y_{2}^{j}-D_{2}\right) 1_{\kappa_{2}^{i} \leq D_{2} \leq y_{2}^{i}+y_{2}^{j}}\right\} \\
= & -c_{2}^{i}+c_{1}^{i}+\left(p_{2}^{i}-c_{1}^{i}\right) \int_{y_{2}^{i}+y_{2}^{j}}^{\infty} f_{2}(u) d u \\
& +\int_{0}^{\kappa_{2}^{i}}\left(\frac{\partial \pi_{1 \mid u}^{i}}{\partial x_{1}^{i}}\left(y_{2}^{i}-q_{2}^{i}(u), y_{2}^{j}-q_{2}^{j}(u)\right)-c_{1}^{i}\right) f_{2}(u) d u \\
& +\int_{\kappa_{2}^{i}}^{y_{2}^{i}+y_{2}^{j}}\left(p_{2}^{i}-c_{1}^{i}+\frac{\partial \pi_{1 \mid u}^{i}}{\partial x_{1}^{j}}\left(0, y_{2}^{i}+y_{2}^{j}-u\right)\right) f_{2}(u) d u .
\end{aligned}
$$

The second integral may not be decreasing in $y_{2}^{i}$, since its variation is related to the second derivative of the profit-to-go with respect to $x_{1}^{j}$, which may not even exist. Using Equation (18), we can express it as

$$
\begin{aligned}
& \int_{\kappa_{2}^{i}}^{y_{2}^{i}+y_{2}^{j}}\left(p_{2}^{i}-c_{1}^{i}+\frac{\partial \pi_{1 \mid u}^{i}}{\partial x^{j}}\left(0, y_{2}^{i}+y_{2}^{j}-u\right)\right) f_{2}(u) d u \\
= & \int_{\kappa_{2}^{i}}^{y_{2}^{i}+y_{2}^{j}}\left[p_{2}^{i}-c_{1}^{i}-p_{1}^{i} 1_{\frac{c_{1}^{i}}{p_{1}^{i}} \leq \bar{F}_{1 \mid u}\left(\left(q_{1}^{j}\right)^{-1}\left(y_{2}^{i}+y_{2}^{j}-u\right)\right) \leq \frac{c_{1}^{j}}{p_{1}^{j}}}\left(\bar{F}_{1 \mid u}\left(\left(q_{1}^{j}\right)^{-1}\left(y_{2}^{i}+y_{2}^{j}-u\right)\right)-\frac{c_{1}^{i}}{p_{1}^{i}}\right)\right] f_{2}(u) d u
\end{aligned}
$$

Let $\bar{G}(x):=\int_{x}^{\infty} g(t) d t$. We thus have $\bar{F}_{1 \mid u}\left(\left(q_{1}^{j}\right)^{-1}\left(y_{2}^{i}+y_{2}^{j}-u\right)\right)=\bar{G}\left(\frac{y_{2}^{i}+y_{2}^{j}-u}{\alpha_{1}^{j}}-k u\right)$.

When $k>0$, consider the change of variables $v=\frac{y_{2}^{i}+y_{2}^{j}-u}{\alpha_{1}^{j}}-k u$, or equivalently $u=\frac{y_{2}^{i}+y_{2}^{j}-\alpha_{1}^{j} v}{1+k \alpha_{1}^{j}}$. We obtain

$$
\begin{aligned}
& \int_{\kappa_{2}^{i}}^{y_{2}^{i}+y_{2}^{j}}\left[p_{2}^{i}-c_{1}^{i}-p_{1}^{i} 1_{\frac{c_{1}^{i}}{p_{1}^{i}} \leq \bar{F}_{1 \mid u}\left(\left(q_{1}^{j}\right)^{-1}\left(y_{2}^{i}+y_{2}^{j}-u\right)\right) \leq \frac{c_{1}^{j}}{p_{1}^{j}}}\left(\bar{F}_{1 \mid u}\left(\left(q_{1}^{j}\right)^{-1}\left(y_{2}^{i}+y_{2}^{j}-u\right)\right)-\frac{c_{1}^{i}}{p_{1}^{i}}\right)\right] f_{2}(u) d u \\
= & \int_{0}^{\frac{y_{2}^{i}+y_{2}^{j}-\kappa_{2}^{i}}{\alpha_{1}^{j}}-k \kappa_{2}^{i}}\left[p_{2}^{i}-c_{1}^{i}-p_{1}^{i} 1_{\frac{c_{1}^{i} \leq \bar{G}(v) \leq \frac{c_{1}^{j}}{p_{1}^{j}}}{p_{1}^{i}}}\left(\bar{G}(v)-\frac{c_{1}^{i}}{p_{1}^{i}}\right)\right] f_{2}\left(\frac{y_{2}^{i}+y_{2}^{j}-\alpha_{1}^{j} v}{1+k \alpha_{1}^{j}}\right)\left(\frac{\alpha_{1}^{j}}{1+k \alpha_{1}^{j}}\right) d v
\end{aligned}
$$


As a result, the use of Leibniz' rule for differentiation yields

$$
\begin{aligned}
& \frac{\partial}{\partial y_{2}^{i}}\left(\int_{\kappa_{2}^{i}}^{y_{2}^{i}+y_{2}^{j}}\left(p_{2}^{i}-c_{1}^{i}+\frac{\partial \pi_{1 \mid u}^{i}}{\partial x^{j}}\left(0, y_{2}^{i}+y_{2}^{j}-u\right)\right) f_{2}(u) d u\right) \\
&=\left\{\begin{array}{l}
{\left[1-\left(\frac{1}{\alpha_{1}^{j}}+k\right) \frac{1}{\left(q_{2}^{i}\right)^{\prime}\left(\kappa_{2}^{i}\right)}\right] \times f_{2}\left(\kappa_{2}^{i}\right) \times\left(\frac{\alpha_{1}^{j}}{1+k \alpha_{1}^{j}}\right)} \\
\times\left[p_{2}^{i}-c_{1}^{i}-p_{1}^{i} 1_{\frac{c_{1}^{i}}{p_{1}^{i}} \leq \bar{G}\left(\frac{y_{2}^{i}+y_{2}^{j}-\kappa_{2}^{i}}{\alpha_{1}^{j}}-k \kappa_{2}^{i}\right) \leq \frac{c_{1}^{j}}{p_{1}^{j}}}\left(\bar{G}\left(\frac{y_{2}^{i}+y_{2}^{j}-\kappa_{2}^{i}}{\alpha_{1}^{j}}-k \kappa_{2}^{i}\right)-\frac{c_{1}^{i}}{p_{1}^{i}}\right)\right]
\end{array}\right\} \\
&+\int_{0}^{\frac{y_{2}^{i}+y_{2}^{j}-\kappa_{2}^{i}}{\alpha_{1}^{j}}-k \kappa_{2}^{i}}\left[p_{2}^{i}-c_{1}^{i}-p_{1}^{i} 1_{\frac{c_{1}^{i}}{p_{1}^{i}} \leq \bar{G}(v) \leq \frac{c_{1}^{j}}{p_{1}^{j}}}\left(\bar{G}(v)-\frac{c_{1}^{i}}{p_{1}^{i}}\right)\right] f_{2}^{\prime}\left(\frac{y_{2}^{i}+y_{2}^{j}-\alpha_{1}^{j} v}{1+k \alpha_{1}^{j}}\right)\left(\frac{\alpha_{1}^{j}}{\left(1+k \alpha_{1}^{j}\right)^{2}}\right) d v
\end{aligned}
$$

Since $0 \leq \alpha_{1}^{j} \leq 1, k \geq 0$ and $\left(q_{2}^{i}\right)^{\prime}\left(\kappa_{2}^{i}\right) \leq 1$, the first term is non-positive. In addition, because of log-concavity of $D_{2}, \frac{f_{2}^{\prime}}{f_{2}}$ is non-increasing and hence for $0 \leq v \leq \frac{y_{2}^{i}+y_{2}^{j}-\kappa_{2}^{i}}{\alpha_{1}^{j}}-k \kappa_{2}^{i}$

$$
\frac{f_{2}^{\prime}}{f_{2}}\left(\frac{y_{2}^{i}+y_{2}^{j}-\alpha_{1}^{j} v}{1+k \alpha_{1}^{j}}\right)\left(\frac{1}{1+k \alpha_{1}^{j}}\right) \leq \frac{f_{2}^{\prime}}{f_{2}}\left(\kappa_{2}^{i}\right)\left(\frac{1}{1+k \alpha_{1}^{j}}\right) \leq \phi_{2}\left(\left(q_{2}^{i}\right)^{-1}\left(y_{2}^{i}\right)\right) .
$$

As a result,

$$
\begin{aligned}
& \frac{\partial}{\partial y_{2}^{i}}\left(\int_{\left(q_{2}^{i}\right)^{-1}\left(y_{2}^{i}\right)}^{y_{2}^{i}+y_{2}^{j}}\left(p_{2}^{i}-c_{1}^{i}+\frac{\partial \pi_{1 \mid u}^{i}}{\partial x^{j}}\left(0, y_{2}^{i}+y_{2}^{j}-u\right)\right) f_{2}(u) d u\right) \\
\leq & \phi_{2}\left(\left(q_{2}^{i}\right)^{-1}\left(y_{2}^{i}\right)\right)\left(\int_{\left(q_{2}^{i}\right)^{-1}\left(y_{2}^{i}\right)}^{y_{2}^{i}+y_{2}^{j}}\left(p_{2}^{i}-c_{1}^{i}+\frac{\partial \pi_{1 \mid u}^{i}}{\partial x^{j}}\left(0, y_{2}^{i}+y_{2}^{j}-u\right)\right) f_{2}(u) d u\right)
\end{aligned}
$$

When $k=0$, i.e., $D_{1}$ is independent from $D_{2}$, then the same result is obtained using the change of variables $v=y_{2}^{i}+y_{2}^{j}-u$, since

$$
\begin{aligned}
& \int_{\kappa_{2}^{i}}^{y_{2}^{i}+y_{2}^{j}}\left[p_{2}^{i}-c_{1}^{i}-p_{1}^{i} 1_{\frac{c_{1}^{i}}{p_{1}^{i}} \leq \bar{F}_{1 \mid u}\left(\left(q_{1}^{j}\right)^{-1}\left(y_{2}^{i}+y_{2}^{j}-u\right)\right) \leq \frac{c_{1}^{j}}{p_{1}^{j}}}\left(\bar{F}_{1 \mid u}\left(\left(q_{1}^{j}\right)^{-1}\left(y_{2}^{i}+y_{2}^{j}-u\right)\right)-\frac{c_{1}^{i}}{p_{1}^{i}}\right)\right] f_{2}(u) d u \\
= & \int_{0}^{y_{2}^{i}+y_{2}^{j}-\kappa_{2}^{i}}\left[p_{2}^{i}-c_{1}^{i}-p_{1}^{i} 1_{\frac{c_{1}^{i}}{p_{1}^{i}} \leq \bar{G}\left(\left(q_{1}^{j}\right)^{-1}(v)\right) \leq \frac{c_{1}^{j}}{p_{1}^{j}}}\left(\bar{G}\left(\left(q_{1}^{j}\right)^{-1}(v)\right)-\frac{c_{1}^{i}}{p_{1}^{i}}\right)\right] f_{2}\left(y_{2}^{i}+y_{2}^{j}-v\right) d v .
\end{aligned}
$$

Note that in this case no assumption on the linearity of $q_{1}^{j}$ is made. 
Thus, differentiating Equation (20) yields

$$
\begin{aligned}
\frac{\partial^{2} r_{2}^{i}}{\left(\partial y_{2}^{i}\right)^{2}}= & -\left(p_{2}^{i}-c_{1}^{i}\right) f_{2}\left(y_{2}^{i}+y_{2}^{j}\right)+\frac{\frac{\partial \pi_{1 \mid \kappa_{2}^{i}}^{i}}{\partial x_{1}^{i}}\left(0, y_{2}^{j}-q_{2}^{j}\left(\kappa_{2}^{i}\right)\right)-c_{1}^{i}}{\left(q_{2}^{i}\right)^{\prime}\left(\kappa_{2}^{i}\right)} f_{2}\left(\kappa_{2}^{i}\right) \\
& +\int_{0}^{\kappa_{2}^{i}} \frac{\partial^{2} \pi_{1 \mid u}^{i}}{\left(\partial x_{1}^{i}\right)^{2}}\left(y_{2}^{i}-q_{2}^{i}(u), y_{2}^{j}-q_{2}^{j}(u)\right) f_{2}(u) d u \\
& +\frac{\partial}{\partial y_{2}^{i}}\left(\int_{\kappa_{2}^{i}}^{y_{2}^{i}+y_{2}^{j}}\left(p_{2}^{i}-c_{1}^{i}+\frac{\partial \pi_{1 \mid u}^{i}}{\partial x^{j}}\left(0, y_{2}^{i}+y_{2}^{j}-u\right)\right) f_{2}(u) d u\right) \\
< & \int_{0}^{\kappa_{2}^{i}} \frac{\partial^{2} \pi_{1 \mid u}^{i}}{\left(\partial x_{1}^{i}\right)^{2}}\left(y_{2}^{i}-q_{2}^{i}(u), y_{2}^{j}-q_{2}^{j}(u)\right) f_{2}(u) d u \\
& +\phi_{2}\left(\left(q_{2}^{i}\right)^{-1}\left(y_{2}^{i}\right)\right)\left(\int_{\left(q_{2}^{i}\right)^{-1}\left(y_{2}^{i}\right)}^{y_{2}^{i}+y_{2}^{j}}\left(p_{2}^{i}-c_{1}^{i}+\frac{\partial \pi_{1 \mid u}^{i}}{\partial x^{j}}\left(0, y_{2}^{i}+y_{2}^{j}-u\right)\right) f_{2}(u) d u\right)
\end{aligned}
$$

where we used $\frac{\partial \pi_{1 \mid \kappa_{2}^{i}}^{i}}{\partial x^{i}}\left(0, y_{2}^{j}-q_{2}^{j}\left(\kappa_{2}^{i}\right)\right)-c_{1}^{i} \leq 0$ from Claim 2 and the infinite support.

Using Claim 1 and assumption (iii) of the proposition, we have that

$$
\begin{aligned}
\frac{\partial^{2} \pi_{1 \mid u}^{i}}{\left(\partial x_{1}^{i}\right)^{2}}\left(y_{2}^{i}-q_{2}^{i}(u), y_{2}^{j}-q_{2}^{j}(u)\right) & \leq \phi_{1 \mid u}\left(\left(q_{1}^{i}\right)^{-1}\left(y_{2}^{i}-q_{2}^{i}(u)\right)\right)\left(\frac{\partial \pi_{1 \mid u}^{i}}{\partial x_{1}^{i}}\left(y_{2}^{i}-q_{2}^{i}(u), y_{2}^{j}-q_{2}^{j}(u)\right)-c_{1}^{i}\right) \\
& \leq \phi_{2}\left(\left(q_{2}^{i}\right)^{-1}\left(y_{2}^{i}-q_{2}^{i}(u)\right)\left(\frac{\partial \pi_{1 \mid u}^{i}}{\partial x_{1}^{i}}\left(y_{2}^{i}-q_{2}^{i}(u), y_{2}^{j}-q_{2}^{j}(u)\right)-c_{1}^{i}\right)\right. \\
& \leq \phi_{2}\left(\left(q_{2}^{i}\right)^{-1}\left(y_{2}^{i}\right)\right)\left(\frac{\partial \pi_{1 \mid u}^{i}}{\partial x_{1}^{i}}\left(y_{2}^{i}-q_{2}^{i}(u), y_{2}^{j}-q_{2}^{j}(u)\right)-c_{1}^{i}\right),
\end{aligned}
$$

where we used that $f_{2}$ is log-concave (so that $\phi_{2}$ is non-increasing). Hence,

$$
\begin{aligned}
\frac{\partial^{2} r_{2}^{i}}{\left(\partial y_{2}^{i}\right)^{2}}< & \phi_{2}\left(\left(q_{2}^{i}\right)^{-1}\left(y_{2}^{i}\right)\right) \int_{0}^{\left(q_{2}^{i}\right)^{-1}\left(y_{2}^{i}\right)}\left(\frac{\partial \pi_{1 \mid u}^{i}}{\partial x^{i}}\left(y_{2}^{i}-q_{2}^{i}(u), y_{2}^{j}-q_{2}^{j}(u)\right)-c_{1}^{i}\right) f_{2}(u) d u \\
& +\phi_{2}\left(\left(q_{2}^{i}\right)^{-1}\left(y_{2}^{i}\right)\right) \int_{\left(q_{2}^{i}\right)^{-1}\left(y_{2}^{i}\right)}^{y_{2}^{i}+y_{2}^{j}}\left(p_{2}^{i}-c_{1}^{i}+\frac{\partial \pi_{1 \mid u}^{i}}{\partial x^{j}}\left(0, y_{2}^{i}+y_{2}^{j}-u\right)\right) f_{2}(u) d u \\
= & \phi_{2}\left(\left(q_{2}^{i}\right)^{-1}\left(y_{2}^{i}\right)\right)\left(\frac{\partial r_{2}^{i}}{\partial y_{2}^{i}}-\left(c_{1}^{i}-c_{2}^{i}\right)-\left(p_{2}^{i}-c_{1}^{i}\right) \int_{y_{2}^{i}+y_{2}^{j}}^{\infty} f_{2}(u) d u\right) \\
\leq & \phi_{2}\left(\left(q_{2}^{i}\right)^{-1}\left(y_{2}^{i}\right)\right)\left(\frac{\partial r_{2}^{i}}{\partial y_{2}^{i}}\right) .
\end{aligned}
$$

This ends the proof of the third claim, and therefore, the proof of the proposition is also complete.

\section{Proof of Theorem 3}

Proof. We use first the following claim.

Claim $4 \frac{\partial^{2} \pi_{1}^{i}}{\left(\partial x_{1}^{i}\right)^{2}} \leq \frac{\partial^{2} \pi_{1}^{i}}{\partial x_{1}^{i} \partial x_{1}^{j}} \leq 0$ almost everywhere. 
Note that these quantities are well-defined almost everywhere because, as seen in the proof of Proposition $1, \frac{\partial \pi_{1}^{i}}{\partial x_{1}^{i}}$ is continuous, and the points of non-differentiability are at the borders of regions (I)-(IV) only. The first inequality is easily proved using that $\frac{\partial^{2} r_{1}}{\left(\partial y_{1}^{i}\right)^{2}} \leq \frac{\partial^{2} r_{1}}{\partial y_{1}^{i} \partial y_{1}^{j}}$. The second part comes from the fact that, in each region, the cross-derivative is either zero, in regions (I)-(III), or non-positive in region (IV).

Similarly as in the previous proofs, let $\kappa_{2}^{i}\left(y_{2}^{i}\right)=\left(q_{2}^{i}\right)^{-1}\left(y_{2}^{i}\right)$ and $\kappa_{2}^{j}\left(y_{2}^{j}\right)=\left(q_{2}^{j}\right)^{-1}\left(y_{2}^{j}\right)$. When $\kappa_{2}^{i} \geq \kappa_{2}^{j}$

$$
\frac{\partial^{2} r_{2}^{i}}{\left(\partial y_{2}^{i}\right)^{2}}-\frac{\partial^{2} r_{2}^{i}}{\partial y_{2}^{i} \partial y_{2}^{j}}=\mathbb{E}\left\{\left[\frac{\partial^{2} \pi_{1 \mid D_{2}}^{i}}{\left(\partial x_{1}^{i}\right)^{2}}-\frac{\partial^{2} \pi_{1 \mid D_{2}}^{i}}{\partial x_{1}^{i} \partial x_{1}^{j}}\right]\left(y_{2}^{i}-q_{2}^{i}\left(D_{2}\right), y_{2}^{j}-q_{2}^{j}\left(D_{2}\right)\right) 1_{\kappa_{2}^{j} \geq D_{2}}\right\} \leq 0
$$

and

$$
\begin{aligned}
\frac{\partial^{2} r_{2}^{i}}{\partial y_{2}^{i} \partial y_{2}^{j}}= & -\left(p_{2}^{i}-\frac{\partial \pi_{1 \mid D_{2}}^{i}}{\partial x_{1}^{i}}(0,0)\right) f_{2}\left(y_{2}^{i}+y_{2}^{j}\right)+\mathbb{E}\left\{\frac{\partial^{2} \pi_{1 \mid D_{2}}^{i}}{\partial x_{1}^{i} \partial x_{1}^{j}}\left(y_{2}^{i}-q_{2}^{i}\left(D_{2}\right), y_{2}^{j}-q_{2}^{j}\left(D_{2}\right)\right) 1_{\kappa_{2}^{j} \geq D_{2}}\right\} \\
& +\mathbb{E}\left\{\frac{\partial^{2} \pi_{1 \mid D_{2}}^{i}}{\left(\partial x_{1}^{i}\right)^{2}}\left(y_{2}^{i}+y_{2}^{j}-D_{2}, 0\right) 1_{\left(q_{2}^{j}\right)^{-1}\left(y_{2}^{j}\right) \leq D_{2} \leq y_{2}^{i}+y_{2}^{j}}\right\} \\
\leq & 0 .
\end{aligned}
$$

In addition, when $\kappa_{2}^{i}<\kappa_{2}^{j}$,

$$
\begin{aligned}
\frac{\partial^{2} r_{2}^{i}}{\left(\partial y_{2}^{i}\right)^{2}}-\frac{\partial^{2} r_{2}^{i}}{\partial y_{2}^{i} \partial y_{2}^{j}}= & -\frac{p_{2}^{i} f_{2}\left(\kappa_{2}^{i}\right)}{\left(q_{2}^{i}\right)^{\prime}\left(\kappa_{2}^{i}\right)} \\
& +\frac{f_{2}\left(\kappa_{2}^{i}\right)}{\left(q_{2}^{i}\right)^{\prime}\left(\kappa_{2}^{i}\right)}\left(\left[\frac{\partial \pi_{1 \mid \kappa_{2}^{i}}^{i}}{\partial x_{1}^{i}}-\frac{\partial \pi_{1 \mid \kappa_{2}^{i}}^{i}}{\partial x_{1}^{j}}\right]\left(0, y_{2}^{j}+y_{2}^{i}-\kappa_{2}^{i}\right)\right) \\
& +\mathbb{E}\left\{\left[\frac{\partial^{2} \pi_{1 \mid D_{2}}^{i}}{\left(\partial x_{1}^{i}\right)^{2}}-\frac{\partial^{2} \pi_{1 \mid D_{2}}^{i}}{\partial x_{1}^{i} \partial x_{1}^{j}}\right]\left(y_{2}^{i}-q_{2}^{i}\left(D_{2}\right), y_{2}^{j}-q_{2}^{j}\left(D_{2}\right)\right) 1_{\left(q_{2}^{i}\right)^{-1}\left(y_{2}^{i}\right) \geq D_{2}}\right\} \\
< & \frac{f_{2}\left(\kappa_{2}^{i}\right)}{\left(q_{2}^{i}\right)^{\prime}\left(\kappa_{2}^{i}\right)}\left(p_{1}^{i}-p_{2}^{i}\right) \\
\leq & 0
\end{aligned}
$$

because $\frac{\partial \pi_{1 \mid D_{2}}^{i}}{\partial x_{1}^{i}}(0, z)-\frac{\partial \pi_{1 \mid D_{2}}^{i}}{\partial x_{1}^{j}}(0, z)<c_{1}^{i}+\left(p_{i}^{1}-c_{1}^{i}\right)=p_{1}^{i}$ from Claim 2 in the previous proof.

From the implicit function theorem, $\frac{d s_{2}^{i}}{d y_{2}^{j}}=\frac{\frac{\partial^{2} r_{2}^{i}}{\partial y_{2}^{i} \partial y_{2}^{j}}}{-\frac{\partial^{2} r_{2}^{i}}{\left(\partial y_{2}^{i}\right)^{2}}}$. Thus, using Equations (21) and (23), we have that $\frac{d s_{2}^{i}}{d y_{2}^{j}} \geq-1$. In addition, since at each point, either $\kappa_{2}^{i}<\kappa_{2}^{j}$ or the reverse, we must have either $\frac{d s_{2}^{i}}{d y_{2}^{j}} \leq 0$ and $\frac{d s_{2}^{j}}{d y_{2}^{i}}>-1$; or $\frac{d s_{2}^{i}}{d y_{2}^{j}}>-1$ and $\frac{d s_{2}^{j}}{d y_{2}^{i}} \leq 0$. This implies that in 
each point, $\frac{d s_{2}^{i}}{d y_{2}^{j}} \frac{d s_{2}^{j}}{d y_{2}^{i}}<1$

The two best-response functions are continuous. $s_{2}^{i}\left(y_{2}^{j}\right)$ is decreasing for small $y_{2}^{j}$, while $\left(q_{2}^{i}\right)^{-1}\left(s_{2}^{i}\left(y_{2}^{j}\right)\right) \geq\left(q_{2}^{j}\right)^{-1}\left(y_{2}^{j}\right)$. As a result, the two best-response functions intersect in the region $\left[0, s_{2}^{i}(0)\right] \times\left[0, s_{2}^{j}(0)\right]$ : equilibrium exists. In addition, since in any equilibrium point $\frac{d s_{2}^{i}}{d y_{2}^{j}} \frac{d s_{2}^{j}}{d y_{2}^{i}}<1$, equilibrium is unique, see Cachon and Netessine (2004).

\section{Proof of Theorem 4}

Proof. Since retailer $j$ is passive, it is clear that for all $t, s_{t}^{j}\left(y_{t}^{i}\right)=0$. Thus, we need to show that firm $i$ 's policy is a base-stock policy that may depend on $y_{t}^{j}=x_{t}^{j}$. In other words, show that $b_{t}^{i}\left(x_{t}^{i}, y_{t}^{j}\right)=\max \left\{x_{t}^{i}, s_{t}^{j}\left(y_{t}^{j}\right)\right\}$. For this purpose, we follow the lines of proof from Proposition 2. Specifically, we show by induction on $t=1, \ldots, T$, that,

(i) for all $y_{t}^{j}, r_{t}^{i}\left(\cdot, y_{t}^{j}\right)$ is quasi-concave; hence a base-stock policy of level $s_{t}^{i}\left(y_{t}^{j}\right)$ is optimal;

(ii) there is a unique equilibrium: $e_{t}^{j}\left(x_{t}^{j}, x_{t}^{i}\right)=x_{t}^{j}$ and $e_{t}^{i}\left(x_{t}^{i}, x_{t}^{j}\right)=\max \left\{x_{t}^{i}, s_{t}^{i}\left(x_{t}^{j}\right)\right\}$;

(iii) $\pi_{t}^{i}$ is concave in $x_{t}^{i}, \frac{\partial \pi_{t}^{i}}{\partial x_{t}^{i}}\left(x_{t}^{i}, x_{t}^{j}\right) \leq c_{t}^{i}$;

(iv) Denoting

$$
\phi_{t}(y)=\max \left\{0, \frac{f_{t}^{\prime}}{f_{t}}(y)\right\}
$$

we have $\frac{\partial^{2} \pi_{t}^{i}}{\left(\partial x_{t}^{i}\right)^{2}} \leq-\phi_{t}\left(\left(q_{t}^{i}\right)^{-1}\left(x_{t}^{i}\right)\right)\left(c_{t}^{i}-\frac{\partial \pi_{t}^{i}}{\partial x_{t}^{i}}\right) \leq 0$;

(v) $\frac{\partial^{2} \pi_{t}^{i}}{\left(\partial x_{t}^{i}\right)^{2}} \leq \frac{\partial^{2} \pi_{t}^{i}}{\partial x_{t}^{i} \partial x_{t}^{j}}$

(vi) $\frac{\partial \pi_{t}^{i}}{\partial x_{t}^{j}}\left(0, x_{t}^{j}\right) \geq-\left(p_{t}^{i}-c_{t}^{i}\right)$.

(i)-(vi) are true for $t=1$, from the proofs of Proposition 1 and Theorem 3.

Assume that the induction properties are true for $t-1$. For $t$, fix $y_{t}^{j}$. Similarly as in the previous proofs, let $\kappa_{t}^{i}\left(y_{t}^{i}\right)=\left(q_{t}^{i}\right)^{-1}\left(y_{t}^{i}\right)$ and $\kappa_{t}^{j}\left(y_{t}^{j}\right)=\left(q_{t}^{j}\right)^{-1}\left(y_{t}^{j}\right)$. There are two possible situations given $y_{t}^{i}, y_{t}^{j}$ : either $j$ stocks out before $i$, and hence there may be a spill over from $j$ to $i$, i.e., $\kappa_{t}^{i} \geq \kappa_{t}^{j}$; or vice-versa. When $\kappa_{t}^{i} \geq \kappa_{t}^{j}$,

$$
\begin{aligned}
\frac{\partial r_{t}^{i}}{\partial y_{t}^{i}}= & -c_{t}^{i}+p_{t}^{i} \mathbb{P}\left\{y_{t}^{i}+y_{t}^{j} \leq D_{t}\right\}+\mathbb{E}\left\{\frac{\partial \pi_{t-1}^{i}}{\partial x_{t-1}^{i}}\left(y_{t}^{i}-q_{t}^{i}\left(D_{t}\right), y_{t}^{j}-q_{t}^{j}\left(D_{t}\right)\right) 1_{\kappa_{t}^{j} \geq D_{t}}\right\} \\
& +\mathbb{E}\left\{\frac{\partial \pi_{t-1}^{i}}{\partial x_{t-1}^{i}}\left(y_{t}^{i}+y_{t}^{j}-D_{t}, 0\right) 1_{\kappa_{t}^{j} \leq D_{t} \leq y_{t}^{i}+y_{t}^{j}}\right\}
\end{aligned}
$$


Since $\pi_{t-1}^{i}$ is concave in $x_{t-1}^{i}, r_{t}^{i}$ is concave in $y_{t}^{i}$. In fact, the second derivative can be expressed as

$$
\begin{aligned}
\frac{\partial^{2} r_{t}^{i}}{\left(\partial y_{t}^{i}\right)^{2}}= & -p_{t}^{i} f_{t}\left(y_{t}^{i}+y_{t}^{j}\right)+\mathbb{E}\left\{\frac{\partial^{2} \pi_{t-1}^{i}}{\left(\partial x_{t-1}^{i}\right)^{2}}\left(y_{t}^{i}-q_{t}^{i}\left(D_{t}\right), y_{t}^{j}-q_{t}^{j}\left(D_{t}\right)\right) 1_{\kappa_{t}^{j} \geq D_{t}}\right\} \\
& +\mathbb{E}\left\{\frac{\partial^{2} \pi_{t-1}^{i}}{\left(\partial x_{t-1}^{i}\right)^{2}}\left(y_{t}^{i}+y_{t}^{j}-D_{t}, 0\right) 1_{\kappa_{t}^{j} \leq D_{t} \leq y_{t}^{i}+y_{t}^{j}}\right\} \\
\leq & \mathbb{E}\left\{\phi_{t-1}\left(\left(q_{t-1}^{i}\right)^{-1}\left(y_{t}^{i}-q_{t}^{i}\left(D_{t}\right)\right)\right)\left(\frac{\partial \pi_{t-1}^{i}}{\partial x_{t-1}^{i}}\left(y_{t}^{i}-q_{t}^{i}\left(D_{t}\right), y_{t}^{j}-q_{t}^{j}\left(D_{t}\right)\right)-c_{t-1}^{i}\right) 1_{\kappa_{t}^{j} \geq D_{t}}\right\} \\
& +\mathbb{E}\left\{\phi_{t-1}\left(\left(q_{t-1}^{i}\right)^{-1}\left(y_{t}^{i}+y_{t}^{j}-D_{t}\right)\left(\frac{\partial \pi_{t-1}^{i}}{\partial x_{t-1}^{i}}\left(y_{t}^{i}+y_{t}^{j}-D_{t}, 0\right)-c_{t-1}^{i}\right) 1_{\kappa_{t}^{j} \leq D_{t} \leq y_{t}^{i}+y_{t}^{j}}\right\}\right. \\
& (\text { using part (iv) of the induction) } \\
\leq & \mathbb{E}\left\{\phi_{t}\left(\left(q_{t}^{i}\right)^{-1}\left(y_{t}^{i}\right)\right)\left(\frac{\partial \pi_{t-1}^{i}}{\partial x_{t-1}^{i}}\left(y_{t}^{i}-q_{t}^{i}\left(D_{t}\right), y_{t}^{j}-q_{t}^{j}\left(D_{t}\right)\right)-c_{t-1}^{i}\right) 1_{\kappa_{t}^{j} \geq D_{t}}\right\} \\
& +\mathbb{E}\left\{\phi_{t}\left(\left(q_{t}^{i}\right)^{-1}\left(y_{t}^{i}\right)\right)\left(\frac{\partial \pi_{t-1}^{i}}{\partial x_{t-1}^{i}}\left(y_{t}^{i}+y_{t}^{j}-D_{t}, 0\right)-c_{t-1}^{i}\right) 1_{\kappa_{t}^{j} \leq D_{t} \leq y_{t}^{i}+y_{t}^{j}}\right\}
\end{aligned}
$$

(using (iii) of the proposition and that $\phi_{t}$ is non-increasing, from (ii) of the proposition)

$$
\begin{aligned}
& \leq \phi_{t}\left(\left(q_{t}^{i}\right)^{-1}\left(y_{t}^{i}\right)\right)\left(\frac{\partial r_{t}^{i}}{\partial y_{t}^{i}}-\left(c_{t-1}^{i}-c_{t}^{i}\right)-\left(p_{t}^{i}-c_{t-1}^{i}\right) \mathbb{P}\left\{y_{t}^{i}+y_{t}^{j} \leq D_{t}\right\}\right) \\
& \leq \phi_{t}\left(\left(q_{t}^{i}\right)^{-1}\left(y_{t}^{i}\right)\right)\left(\frac{\partial r_{t}^{i}}{\partial y_{t}^{i}}\right) .
\end{aligned}
$$

Also, $\frac{\partial^{2} r_{t}^{i}}{\left(\partial y_{t}^{i}\right)^{2}} \leq \frac{\partial^{2} r_{t}^{i}}{\partial y_{t}^{i} \partial y_{t}^{j}}$, using part (v) of the induction step.

Consider now the second situation, i.e., $\kappa_{t}^{i}<\kappa_{t}^{j}$. Here, since there is only spill-over from $i$ to $j$

$$
\begin{aligned}
\frac{\partial r_{t}^{i}}{\partial y_{t}^{i}}= & -c_{t}^{i}+p_{t}^{i} \mathbb{P}\left\{\kappa_{t}^{i} \leq D_{t}\right\}+\mathbb{E}\left\{\frac{\partial \pi_{t-1}^{i}}{\partial x_{t-1}^{i}}\left(y_{t}^{i}-q_{t}^{i}\left(D_{t}\right), y_{t}^{j}-q_{t}^{j}\left(D_{t}\right)\right) 1_{\kappa_{t}^{i} \geq D_{t}}\right\} \\
& +\mathbb{E}\left\{\frac{\partial \pi_{t-1}^{i}}{\partial x_{t-1}^{j}}\left(0, y_{t}^{i}+y_{t}^{j}-D_{t}\right) 1_{\left.\kappa_{t}^{i} \leq D_{t} \leq y_{t}^{i}+y_{t}^{j}\right\}}\right\} \\
= & -c_{t}^{i}+c_{t-1}^{i}+\left(p_{t}^{i}-c_{t-1}^{i}\right) \int_{y_{t}^{i}+y_{t}^{j}}^{\infty} f_{t}(u) d u \\
& +\int_{0}^{\kappa_{t}^{i}}\left(\frac{\partial \pi_{t-1}^{i}}{\partial x_{t-1}^{i}}\left(y_{t}^{i}-q_{t}^{i}(u), y_{t}^{j}-q_{t}^{j}(u)\right)-c_{t-1}^{i}\right) f_{t}(u) d u \\
& +\int_{\left(q_{t}^{i}\right)^{-1}\left(y_{t}^{i}\right)}^{y_{t}^{i}+y_{t}^{j}}\left(p_{t}^{i}-c_{t-1}^{i}+\frac{\partial \pi_{t-1}^{i}}{\partial x_{t-1}^{j}}\left(0, y_{t}^{i}+y_{t}^{j}-u\right)\right) f_{t}(u) d u \\
= & -c_{t}^{i}+c_{t-1}^{i}+\left(p_{t}^{i}-c_{t-1}^{i}\right) \int_{y_{t}^{i}+y_{t}^{j}}^{\infty} f_{t}(u) d u \\
& +\int_{0}^{\kappa_{t}^{i}}\left(\frac{\partial \pi_{t-1}^{i}}{\partial x_{t-1}^{i}}\left(y_{t}^{i}-q_{t}^{i}(u), y_{t}^{j}-q_{t}^{j}(u)\right)-c_{t-1}^{i}\right) f_{t}(u) d u \\
& +\int_{0}^{y_{t}^{i}+y_{t}^{j}-\kappa_{t}^{i}}\left(p_{t}^{i}-c_{t-1}^{i}+\frac{\partial \pi_{t-1}^{i}}{\partial x_{t-1}^{j}}(0, v)\right) f_{t}\left(y_{t}^{i}+y_{t}^{j}-v\right) d v
\end{aligned}
$$


where we made the change of variables $v=y_{t}^{i}+y_{t}^{j}-u$ in the last integral. First, observe that $\frac{\partial^{2} r_{t}^{i}}{\left(\partial y_{t}^{i}\right)^{2}} \leq \frac{\partial^{2} r_{t}^{i}}{\partial y_{t}^{i} \partial y_{t}^{j}}$. Moreover, differentiation yields

$$
\begin{aligned}
\frac{\partial^{2} r_{t}^{i}}{\left(\partial y_{t}^{i}\right)^{2}}= & -f_{t}\left(y_{t}^{i}+y_{t}^{j}\right)\left(p_{t}^{i}-c_{t-1}^{i}\right) \\
& +\frac{f_{t}\left(\kappa_{t}^{i}\right)}{\left(q_{t}^{i}\right)^{\prime}\left(\kappa_{t}^{i}\right)}\left(\frac{\partial \pi_{t-1}^{i}}{\partial x_{t-1}^{i}}\left(0, y_{t}^{i}+y_{t}^{j}-\kappa_{t}^{i}\right)-c_{t-1}^{i}\right) \\
& +\left(1-\frac{1}{\left(q_{t}^{i}\right)^{\prime}\left(\kappa_{t}^{i}\right)}\right) f_{t}\left(\kappa_{t}^{i}\right)\left(p_{t}^{i}-c_{t-1}^{i}+\frac{\partial \pi_{t-1}^{i}}{\partial x_{t-1}^{j}}\left(0, y_{t}^{i}+y_{t}^{j}-\kappa_{t}^{i}\right)\right) \\
& +\int_{0}^{\kappa_{t}^{i}} \frac{\partial^{2} \pi_{t-1}^{i}}{\left(\partial x_{t-1}^{i}\right)^{2}}\left(y_{t}^{i}-q_{t}^{i}(u), y_{t}^{j}-q_{t}^{j}(u)\right) f_{t}(u) d u \\
& +\int_{0}^{y_{t}^{i}+y_{t}^{j}-\kappa_{t}^{i}}\left(p_{t}^{i}-c_{t-1}^{i}+\frac{\partial \pi_{t-1}^{i}}{\partial x_{t-1}^{j}}(0, v)\right) f_{t}^{\prime}\left(y_{t}^{i}+y_{t}^{j}-v\right) d v \\
\leq & \int_{0}^{\kappa_{t}^{i}} \frac{\partial^{2} \pi_{t-1}^{i}}{\left(\partial x_{t-1}^{i}\right)^{2}}\left(y_{t}^{i}-q_{t}^{i}(u), y_{t}^{j}-q_{t}^{j}(u)\right) f_{t}(u) d u \\
& +\int_{0}^{y_{t}^{i}+y_{t}^{j}-\kappa_{t}^{i}}\left(p_{t}^{i}-c_{t-1}^{i}+\frac{\partial \pi_{t-1}^{i}}{\partial x_{t-1}^{j}}(0, v)\right) f_{t}^{\prime}\left(y_{t}^{i}+y_{t}^{j}-v\right) d v
\end{aligned}
$$

where we used part (iii) and (vi) of the induction step, i.e., $\frac{\partial \pi_{t-1}^{i}}{\partial x_{t-1}^{i}}-c_{t-1}^{i} \leq 0$, and $p_{t}^{i}-c_{t-1}^{i}+\frac{\partial \pi_{t-1}^{i}}{\partial x_{t-1}^{j}}(0, \cdot) \geq 0$. In addition, from the log-concavity of $D_{t}, \frac{f_{t}^{\prime}}{f_{t}}$ is non-increasing and so is $\phi_{t}$. Hence for $v \in\left[0, y_{t}^{i}+y_{t}^{j}-\kappa_{t}^{i}\right]$,

$$
\frac{f_{t}^{\prime}}{f_{t}}\left(y_{t}^{i}+y_{t}^{j}-v\right) \leq \frac{f_{t}^{\prime}}{f_{t}}\left(\kappa_{t}^{i}\right) \leq \phi_{t}\left(\left(q_{t}^{i}\right)^{-1}\left(y_{t}^{i}\right)\right)
$$

Finally, from part (iv) of the induction, for $u \in\left[0,\left(q_{t}^{i}\right)^{-1}\left(y_{t}^{i}\right)\right]$,

$$
\begin{aligned}
& \frac{\partial^{2} \pi_{t-1}^{i}}{\left(\partial x_{t-1}^{i}\right)^{2}}\left(y_{t}^{i}-q_{t}^{i}(u), y_{t}^{j}-q_{t}^{j}(u)\right) \\
\leq & \phi_{t-1}\left(\left(q_{t-1}^{i}\right)^{-1}\left(y_{t}^{i}-q_{t}^{i}(u)\right)\right)\left(\frac{\partial \pi_{t-1}^{i}}{\partial x_{t-1}^{i}}\left(y_{t}^{i}-q_{t}^{i}(u), y_{t}^{j}-q_{t}^{j}(u)\right)-c_{t-1}^{i}\right) \\
\leq & \phi_{t-1}\left(\left(q_{t-1}^{i}\right)^{-1}\left(y_{t}^{i}\right)\right)\left(\frac{\partial \pi_{t-1}^{i}}{\partial x_{t-1}^{i}}\left(y_{t}^{i}-q_{t}^{i}(u), y_{t}^{j}-q_{t}^{j}(u)\right)-c_{t-1}^{i}\right)
\end{aligned}
$$

(because the term in parentheses is non-positive)

$\leq \phi_{t}\left(\left(q_{t}^{i}\right)^{-1}\left(y_{t}^{i}\right)\right)\left(\frac{\partial \pi_{t-1}^{i}}{\partial x_{t-1}^{i}}\left(y_{t}^{i}-q_{t}^{i}(u), y_{t}^{j}-q_{t}^{j}(u)\right)-c_{t-1}^{i}\right)$

(from assumption (iii) of the proposition) 
As a result,

$$
\begin{aligned}
& \frac{\partial^{2} r_{t}^{i}}{\left(\partial y_{t}^{i}\right)^{2}} \leq \phi_{t}\left(\left(q_{t}^{i}\right)^{-1}\left(y_{t}^{i}\right)\right)\left(\begin{array}{c}
\int_{0}^{\kappa_{t}^{i}}\left(\frac{\partial \pi_{t-1}^{i}}{\partial x_{t-1}^{i}}\left(y_{t}^{i}-q_{t}^{i}(u), y_{t}^{j}-q_{t}^{j}(u)\right)-c_{t-1}^{i}\right) f_{t}(u) d u \\
+\int_{0}^{y_{t}^{i}+y_{t}^{j}-\kappa_{t}^{i}}\left(p_{t}^{i}-c_{t-1}^{i}+\frac{\partial \pi_{t-1}^{i}}{\partial x_{t-1}^{j}}(0, v)\right) f_{t}\left(y_{t}^{i}+y_{t}^{j}-v\right) d v
\end{array}\right) \\
& \leq \phi_{t}\left(\left(q_{t}^{i}\right)^{-1}\left(y_{t}^{i}\right)\right)\left(\frac{\partial r_{t}^{i}}{\partial y_{t}^{i}}-\left(c_{t-1}^{i}-c_{t}^{i}\right)-\left(p_{t}^{i}-c_{t-1}^{i}\right) \int_{y_{t}^{i}+y_{t}^{j}}^{\infty} f_{t}(u) d u\right) \\
& \leq \phi_{t}\left(\left(q_{t}^{i}\right)^{-1}\left(y_{t}^{i}\right)\right)\left(\frac{\partial r_{t}^{i}}{\partial y_{t}^{i}}\right) \text {. }
\end{aligned}
$$

Consider a critical point of $r_{t}^{i}\left(\cdot, y_{t}^{j}\right)$, i.e., $\frac{\partial r_{t}^{i}}{\partial y_{t}^{i}}\left(y_{t}^{i}, y_{t}^{j}\right)=0$. We have shown that this is necessarily a maximum, i.e., $\frac{\partial^{2} r_{t}^{i}}{\left(\partial y_{t}^{i}\right)^{2}}\left(y_{t}^{i}, y_{t}^{j}\right) \leq 0$. This proves that $r_{t}^{i}\left(\cdot, y_{t}^{j}\right)$ is first increasing and then decreasing, i.e., quasi-concave. Since, $r_{t}^{i}$ is eventually decreasing for large $y_{t}^{i}$, there is a unique unconstrained maximizer, $s_{t}^{i}\left(y_{t}^{j}\right)$, and the optimal policy is base-stock: $b_{t}^{i}\left(x_{t}^{i}, y_{t}^{j}\right)=\max \left\{x_{t}^{i}, s_{t}^{i}\left(y_{t}^{j}\right)\right\}$. This proves part (i) of the induction. Part (ii) is shown recalling that retailer $\mathrm{j}$ does not order at all: $e_{t}^{j}=x_{t}^{j}$, and thus $e_{t}^{i}=\max \left\{x_{t}^{i}, s_{t}^{i}\left(x_{t}^{j}\right)\right\}$.

Hence, there are two regions to consider:

$$
\pi_{t}^{i}\left(x_{t}^{i}, x_{t}^{j}\right)= \begin{cases}c_{t}^{i} x_{t}^{i}+r_{t}^{i}\left(s_{t}^{i}\left(x_{t}^{j}\right), x_{t}^{j}\right) & \text { for } x_{t}^{i} \leq s_{t}^{i}\left(x_{t}^{j}\right) \\ c_{t}^{i} x_{t}^{i}+r_{t}^{i}\left(x_{t}^{i}, x_{t}^{j}\right) & \text { otherwise. }\end{cases}
$$

In the first region (replenishment, first line in the expression above), $\frac{\partial \pi_{t}^{i}}{\partial x_{t}^{i}}=c_{t}^{i}$ and hence $\frac{\partial^{2} \pi_{t}^{i}}{\left(\partial x_{t}^{i}\right)^{2}} \leq 0=-\phi_{t}\left(\left(q_{t}^{i}\right)^{-1}\left(y_{t}^{i}\right)\right)\left(c_{t}^{i}-\frac{\partial \pi_{t}^{i}}{\partial x_{t}^{i}}\right)$. Also, $\frac{\partial^{2} \pi_{t}^{i}}{\left(\partial x_{t}^{i}\right)^{2}}=\frac{\partial^{2} \pi_{t}^{i}}{\partial x_{t}^{i} \partial x_{t}^{j}}=0$.

In the second region, $\frac{\partial \pi_{t}^{i}}{\partial x_{t}^{i}}=c_{t}^{i}+\frac{\partial r_{t}^{i}}{\partial y_{t}^{i}} \leq c_{t}^{i}$, because $x_{t}^{i} \geq s_{t}^{i}\left(x_{t}^{j}\right)$. In addition, from Equations (25) and (26),

$$
\frac{\partial^{2} \pi_{t}^{i}}{\left(\partial x_{t}^{i}\right)^{2}}=\frac{\partial^{2} r_{t}^{i}}{\left(\partial y_{t}^{i}\right)^{2}} \leq \phi_{t}\left(\left(q_{t}^{i}\right)^{-1}\left(x_{t}^{i}\right)\right)\left(\frac{\partial r_{t}^{i}}{\partial y_{t}^{i}}\right)=-\phi_{t}\left(\left(q_{t}^{i}\right)^{-1}\left(x_{t}^{i}\right)\right)\left(c_{t}^{i}-\frac{\partial \pi_{t}^{i}}{\partial x_{t}^{i}}\right)
$$

Also, $\frac{\partial^{2} \pi_{t}^{i}}{\left(\partial x_{t}^{i}\right)^{2}} \leq \frac{\partial^{2} \pi_{t}^{i}}{\partial x_{t}^{i} \partial x_{t}^{j}}$ since $\frac{\partial^{2} r_{t}^{i}}{\left(\partial y_{t}^{i}\right)^{2}} \leq \frac{\partial^{2} r_{t}^{i}}{\partial y_{t}^{i} \partial y_{t}^{j}}$ (shown before).

Hence, $\pi_{t}^{i}$ is concave in $x_{t}^{i}$, and parts (iii)-(v) are shown. Finally, from the envelope theorem,

$$
\frac{\partial \pi_{t}^{i}}{\partial x_{t}^{j}}\left(0, x_{t}^{j}\right)=\frac{\partial r_{t}^{i}}{\partial y_{t}^{j}}\left(s_{t}^{i}\left(x_{t}^{j}\right), x_{t}^{j}\right)
$$


0 ,

Interestingly, since $\frac{\partial^{2} r_{t}^{i}}{\left(\partial y_{t}^{i}\right)^{2}} \leq \frac{\partial^{2} r_{t}^{i}}{\partial y_{t}^{i} \partial y_{t}^{j}}, \frac{\partial r_{t}^{i}}{\partial y_{t}^{i}}-\frac{\partial r_{t}^{i}}{\partial y_{t}^{j}}$ is non-increasing in $y_{t}^{i}$. Since $\frac{\partial r_{t}^{i}}{\partial y_{t}^{i}}\left(s_{t}^{i}\left(x_{t}^{j}\right), x_{t}^{j}\right)=$

$$
\frac{\partial r_{t}^{i}}{\partial y_{t}^{i}}\left(0, x_{t}^{j}\right)-\frac{\partial r_{t}^{i}}{\partial y_{t}^{j}}\left(0, x_{t}^{j}\right)=p_{t}^{i}-c_{t}^{i} \geq \frac{\partial r_{t}^{i}}{\partial y_{t}^{i}}\left(s_{t}^{i}\left(x_{t}^{j}\right), x_{t}^{j}\right)-\frac{\partial r_{t}^{i}}{\partial y_{t}^{j}}\left(s_{t}^{i}\left(x_{t}^{j}\right), x_{t}^{j}\right)=-\frac{\partial \pi_{t}^{i}}{\partial x_{t}^{j}}\left(0, x_{t}^{j}\right)
$$

This shows part (vi) and completes the induction.

\section{Proof of Theorem 5}

Proof. In the proof, we use the dynamic program presented in Equation (13). This is a standard inventory problem, and it is easy to show that for all $t$, for all possible past information $I_{t}, U_{t \mid I_{t}}$ is concave. It follows that the optimal policy is a base-stock policy with level $s_{t \mid I_{t}}$, where $s_{t \mid I_{t}}$ satisfies

$$
c_{t}=\mathbb{E}\left\{p_{t} 1_{s_{t \mid I_{t}} \leq D_{t}}+\frac{d U_{t-1 \mid I_{t-1}}}{d x_{t-1}}\left(s_{t \mid I_{t}}-D_{t}\right) 1_{s_{t \mid I_{t}} \geq D_{t}} \mid I_{t}\right\}
$$

We show by induction on $t=1, \ldots, T$ that for all $I_{t}$ (which we omit as a subscript below, i.e., we write subscript " $t$ " instead of " $\left.t \mid I_{t} "\right)$,

(i) for all $y_{t}^{j}, r_{t}^{i}\left(\cdot, y_{t}^{j}\right)$ is concave and $\frac{\partial^{2} r_{t}^{i}}{\left(\partial y_{t}^{i}\right)^{2}} \leq \frac{\partial^{2} r_{t}^{i}}{\partial y_{t}^{i} \partial y_{t}^{j}} \leq 0$;

(ii) $s_{t}^{i}\left(y_{t}^{j}\right)$ is non-increasing in $y_{t}^{j}$ and when $\frac{s_{t}^{i}\left(y_{t}^{j}\right)}{\alpha^{i}} \leq \frac{y_{t}^{j}}{\alpha^{j}}, s_{t}^{i}\left(y_{t}^{j}\right)=\alpha^{i} s_{t}$;

(iii) we have existence and uniqueness of equilibrium and for $i=1,2, e_{t}^{i}\left(x_{t}^{i}, x_{t}^{j}\right)=\max \left\{x_{t}^{i}, \alpha^{i} s_{t}\right\}$;

(iv) when $\frac{x_{t}^{i}}{\alpha^{i}} \leq \frac{x_{t}^{j}}{\alpha^{j}}$, then $\pi_{t}^{i}\left(x_{t}^{i}, x_{t}^{j}\right)=\alpha^{i} U_{t}\left(\frac{x_{t}^{i}}{\alpha^{i}}\right)$;

(v) $\frac{\partial^{2} \pi_{t}^{i}}{\left(\partial x_{t}^{i}\right)^{2}} \leq \frac{\partial^{2} \pi_{t}^{i}}{\partial x_{t}^{i} \partial x_{t}^{j}} \leq 0$

For $t=1$, fix $I_{1}$ (and hence omitted below). We know that $r_{1}^{i}$ is concave in $y_{1}^{i}$ for all $y_{1}^{j}$. From Table 3, it is clear that $\frac{\partial^{2} r_{1}^{i}}{\left(\partial y_{1}^{i}\right)^{2}} \leq \frac{\partial^{2} r_{1}^{i}}{\partial y_{1}^{i} \partial y_{1}^{j}} \leq 0$, point (i). Point (ii) follows from Theorem 1: for $\frac{y_{1}^{j}}{\alpha^{j}} \geq \frac{y_{1}^{i}}{\alpha^{i}}, \mathbb{P}\left\{y_{1}^{i} \leq R_{1}^{i}\left(y_{1}^{j}\right)\right\}=\mathbb{P}\left\{y_{1}^{i} \leq \alpha^{i} D_{1}\right\}$, and hence $s_{1}^{i}\left(y_{1}^{j}\right)=\alpha^{i} s_{1}$.

Since the best-response functions are non-increasing with slope greater than -1 , a Nash equilibrium exists (Theorem 1 again); and since either $s_{1}^{i}\left(y_{1}^{j}\right)$ or $s_{1}^{j}\left(y_{1}^{i}\right)$ are constant (depending on $\frac{y_{1}^{j}}{\alpha^{j}} \geq \frac{y_{1}^{i}}{\alpha^{i}}$ or not), then the equilibrium is unique. In addition, it is easy to check that $E_{1}^{i}:=e_{1}^{i}(0,0)=\alpha^{i} s_{1}$ and $E_{1}^{j}:=e_{1}^{j}(0,0)=\alpha^{j} s_{1}$, and that above the equilibrium level $E_{1}^{j}$, $s_{1}^{i}\left(e_{1}^{j}\right)$ is constant equal to $\alpha^{i} s_{1}$. This implies $e_{1}^{i}=\max \left\{x_{1}^{i}, \alpha^{i} s_{1}\right\}$, point (iii). 
In particular, if $\frac{x_{1}^{i}}{\alpha^{i}} \leq \frac{x_{1}^{j}}{\alpha^{j}}$, then $\frac{e_{1}^{i}}{\alpha^{i}} \leq \frac{e_{1}^{j}}{\alpha^{j}}$. This implies that there is no spill-over from retailer $j$ 's demand into $i$ 's: $\min \left\{e_{1}^{i}, R_{1}^{i}\left(e_{1}^{j}\right)\right\}=\min \left\{e_{1}^{i}, \alpha^{i} D_{1}\right\}$. Hence,

$$
\pi_{1}^{i}\left(x_{1}^{i}, x_{1}^{j}\right)=-c_{1}\left(\max \left\{x_{1}^{i}, \alpha^{i} s_{1}\right\}-x_{1}^{i}\right)+p_{1} \mathbb{E} \min \left\{\max \left\{x_{1}^{i}, \alpha^{i} s_{1}\right\}, \alpha^{i} D_{1}\right\}=\alpha^{i} U_{1}\left(\frac{x_{1}^{i}}{\alpha^{i}}\right),
$$

point (iv). Finally, since

$$
\pi_{1}^{i}\left(x_{1}^{i}, x_{1}^{j}\right)=c_{1}^{i} x_{1}^{i}+r_{1}^{i}\left(\max \left\{x_{1}^{i}, \alpha^{i} s_{1}\right\}, \max \left\{x_{1}^{j}, \alpha^{j} s_{1}\right\}\right)
$$

it is clear that $\frac{\partial^{2} \pi_{1}^{i}}{\left(\partial x_{1}^{i}\right)^{2}} \leq \frac{\partial^{2} \pi_{1}^{i}}{\partial x_{1}^{i} \partial x_{1}^{j}} \leq 0$, point $(\mathrm{v})$. Thus the inductive property is proved for $t=1$.

Assuming that it is true for $t-1$, for all $I_{t-1}$, fix $I_{t}$ (omitted as subscript below).

$$
r_{t}^{i}\left(y_{t}^{i}, y_{t}^{j}\right)=-c_{t} y_{t}^{i}+p_{t} \mathbb{E} \min \left\{y_{t}^{i}, R_{t}^{i}\left(y_{t}^{j}\right)\right\}+\mathbb{E} \pi_{t-1 \mid I_{t-1}}\left(\left(y_{t}^{i}-R_{t}^{i}\left(y_{t}^{j}\right)\right)^{+},\left(y_{t}^{j}-R_{t}^{j}\left(y_{t}^{i}\right)\right)^{+}\right)
$$

and hence

$$
\frac{\partial r_{t}^{i}}{\partial y_{t}^{i}}=-c_{t}+p_{t} \mathbb{P}\left\{y_{t}^{i} \leq R_{t}^{i}\left(y_{t}^{j}\right)\right\}+\mathbb{E} \frac{\partial}{\partial y_{t}^{i}} \pi_{t-1 \mid I_{t-1}}^{i}\left(\left(y_{t}^{i}-R_{t}^{i}\left(y_{t}^{j}\right)\right)^{+},\left(y_{t}^{j}-R_{t}^{j}\left(y_{t}^{i}\right)\right)^{+}\right)
$$

We have that

$$
\begin{aligned}
& \pi_{t-1 \mid I_{t-1}}^{i}\left(\left(y_{t}^{i}-R_{t}^{i}\left(y_{t}^{j}\right)\right)^{+},\left(y_{t}^{j}-R_{t}^{j}\left(y_{t}^{i}\right)\right)^{+}\right) \\
= & \begin{cases}\text {either } & \pi_{t-1 \mid I_{t-1}}^{i}\left(y_{t}^{i}+y_{t}^{j}-D_{t}, 0\right) \\
\text { or } & \pi_{t-1 \mid I_{t-1}}^{i}\left(0, y_{t}^{i}+y_{t}^{j}-D_{t}\right)=\alpha^{i} U_{t-1 \mid I_{t-1}}(0) \\
\text { or } & \pi_{t-1 \mid I_{t-1}}^{i}\left(y_{t}^{i}-\alpha^{i} D_{t}, y_{t}^{j}-\alpha^{j} D_{t}\right) \\
\text { or } & \pi_{t-1 \mid I_{t-1}}^{i}(0,0)=\alpha^{i} U_{t-1 \mid I_{t-1}}(0)\end{cases}
\end{aligned}
$$

Using point (v) of the induction step on $\pi_{t-1 \mid I_{t-1}}^{i}$, we have that $\frac{\partial^{2} r_{t}^{i}}{\left(\partial y_{t}^{i}\right)^{2}} \leq \frac{\partial^{2} r_{t}^{i}}{\partial y_{t}^{i} \partial y_{t}^{j}} \leq 0$, point (i).

This implies that the optimal policy is a base-stock policy with base-stock level $s_{t}^{i}\left(y_{t}^{j}\right)$, and from the implicit function theorem and point (i), we have that $s_{t}^{i}\left(y_{t}^{j}\right)$ is non-increasing. In addition, if $\frac{y_{t}^{i}}{\alpha^{i}} \leq \frac{y_{t}^{j}}{\alpha^{j}}$, there is no spill-over from $j$ to $i$, and hence $\min \left\{y_{t}^{i}, R_{t}^{i}\left(y_{t}^{j}\right)\right\}=$ $\min \left\{y_{t}^{i}, \alpha^{i} D_{t}\right\}$ and

$$
\pi_{t-1 \mid I_{t-1}}^{i}\left(\left(y_{t}^{i}-R_{t}^{i}\left(y_{t}^{j}\right)\right)^{+},\left(y_{t}^{j}-R_{t}^{j}\left(y_{t}^{i}\right)\right)^{+}\right)=\alpha^{i} U_{t-1 \mid I_{t-1}}\left(\frac{\left(y_{t}^{i}-\alpha^{i} D_{t}\right)^{+}}{\alpha^{i}}\right),
$$

from point (iv) of the induction. Hence, when $\frac{s_{t}^{i}\left(y_{t}^{j}\right)}{\alpha^{i}} \leq \frac{y_{t}^{j}}{\alpha^{j}}, s_{t}^{i}\left(y_{t}^{j}\right)=\alpha^{i} s_{t}$. This shows (ii). 
Again, since the best-response functions are non-increasing and with slopes greater than -1 , there exists a Nash equilibrium; as one of the best-response functions is constant, this equilibrium is unique. Similarly as before, $e_{t}^{i}=\max \left\{x_{t}^{i}, \alpha^{i} s_{t}\right\}$ since the best-response function $s_{t}^{i}\left(y_{t}^{j}\right)$ is flat when $y_{t}^{j} \geq \alpha^{j} s_{t}$. This shows (iii).

Again, if $\frac{x_{t}^{i}}{\alpha^{i}} \leq \frac{x_{t}^{j}}{\alpha^{j}}$, then $\frac{e_{t}^{i}}{\alpha^{i}} \leq \frac{e_{t}^{j}}{\alpha^{j}}$. We then have that $\min \left\{e_{t}^{i}, R_{t}^{i}\left(e_{t}^{j}\right)\right\}=\min \left\{e_{t}^{i}, \alpha^{i} D_{t}\right\}$. Hence,

$$
\begin{aligned}
\pi_{t}^{i}\left(x_{t}^{i}, x_{t}^{j}\right)= & -c_{t}\left(\max \left\{x_{t}^{i}, \alpha^{i} s_{t}\right\}-x_{t}^{i}\right)+p_{t} \mathbb{E} \min \left\{\max \left\{x_{t}^{i}, \alpha^{i} s_{t}\right\}, \alpha^{i} D_{t}\right\} \\
& +\mathbb{E} \alpha^{i} U_{t-1 \mid I_{t-1}}\left(\frac{\left(\max \left\{x_{t}^{i}, \alpha^{i} s_{t}\right\}-\alpha^{i} D_{t}\right)^{+}}{\alpha^{i}}\right) \\
= & \alpha^{i} U_{t}\left(\frac{x_{t}^{i}}{\alpha^{i}}\right)
\end{aligned}
$$

point (iv). Finally, since

$$
\pi_{t}^{i}\left(x_{t}^{i}, x_{t}^{j}\right)=c_{t}^{i} x_{t}^{i}+r_{t}^{i}\left(\max \left\{x_{t}^{i}, \alpha^{i} s_{t}\right\}, \max \left\{x_{t}^{j}, \alpha^{j} s_{t}\right\}\right),
$$

it is clear that $\frac{\partial^{2} \pi_{t}^{i}}{\left(\partial x_{t}^{i}\right)^{2}} \leq \frac{\partial^{2} \pi_{t}^{i}}{\partial x_{t}^{i} \partial x_{t}^{j}} \leq 0$, using part (v) of the induction step. This proves point (v) for $t$, for all $I_{t}$, completes the induction and proves the theorem.

\section{References}

Bagnoli, M. and T. Bergstrom. 2005. "Log-Concave Probability and Its Applications." Economic Theory, 26, pp. 445469.

Cachon, G. and S. Netessine. 2004. "Game Theory in Supply Chain Analysis." Supply Chain Analysis in the eBusiness Era, edited by Simchi-Levi D., S. D. Wu and Z.-J. Shen and published by Kluwer Academic Publishers.

Martínez-de-Albéniz, V. 2004. "Portfolio Strategies in Supply Contracts." Ph.D. dissertation, OR Center, MIT. 